Aus der Klinik für Gastroenterologie und Endokrinologie (Prof. Dr. med. V. Ellenrieder)

der Medizinischen Fakultät der Universität Göttingen

\title{
Effekte der Therapie mit Somatostatin-Analoga bei neuroendokrinen Tumoren
}

\author{
INAUGURAL-DISSERTATION \\ zur Erlangung des Doktorgrades \\ der Medizinischen Fakultät der \\ Georg-August-Universität zu Göttingen
}

vorgelegt von

Lena Mirjam Bresch

aus Hechingen 
Dekan:

I. Berichterstatter:

II. Berichterstatter/in:

III. Berichterstatter/in:
Prof. Dr. rer. nat. H. K. Kroemer

Prof. Dr. med. D. Raddatz

PD Dr. med. Kia Homayounfar

Prof. Dr. med. Hans-Ulrich Schildhaus

26.07.2016 


\section{Inhaltsverzeichnis}

Abkürzungsverzeichnis 4

1 Einleitung 7

1.1 Neuroendokrine Tumoren 7

1.1.1 Definition 7

1.1.2 Klassifikation 7

1.1.3 Grading 9

1.1.4 Staging 10

1.2 Diagnostik 13

1.2.1 Klinische Symptomatik 13

1.2.2 Biomarker $\quad 13$

1.2.3 Endoskopie $\quad 15$

1.2.4 Radiologische und nuklearmedizinische Untersuchung 16

1.3 Therapie 17

1.3.1 Chirurgische Therapie 18

1.3.2 Biotherapie mit SSA $\quad 19$

1.3.3 Biotherapie mit Interferon- $\alpha \quad 23$

1.3.4 Therapie mit neuen Pharmaka 23

1.3.5 Chemotherapie 24

1.3.6 Radiotherapie $\quad 25$

1.3.7 Unterstützende symptomatische Therapie 26

1.4 Prognose 26

1.5 Zielsetzung der Arbeit

27

2 Methodik 29

2.1 Klassifikation $\quad 29$

2.1.1 Grading 29

2.1.2 Staging 30

2.2 Diagnostik 31

2.3 Verlaufskontrolle 31

2.3.1 Tumormarker 31

2.3.2 Verlauf der Tumorgröße 32

2.4 Wirksamkeit von SAA 34

2.5 Auswertung 36

3 Ergebnisse 37

3.1 Demographie $\quad 37$

3.1.1 Tumorlokalisation38

3.1.2 Grading bei Erstdiagnose 39

3.1.3 Staging bei Erstdiagnose 39

3.1.4 Funktionelle / nicht funktionelle Tumoren 39 
3.2 Beobachtungsdauer 43

3.2.1 Aus der Analyse ausgeschiedene Patienten

3.3 Therapie mit SAA 44

3.3.1 Therapiedauer der SAA-Monotherapie $\quad 45$

3.3.2 Gesamtdauer der Therapie mit SAA $\quad 45$

3.3.3 Gesamtdauer der Zeitspanne ohne SAA 46

3.3.4 Verlauf der Symptomatik 47

3.3.5 Verlauf der Tumormarker 50

3.3.6 Stable disease unter SAA 55

3.3.6.1 Dauer der stable disease nach Metastasierung bei Erstdiagnose geordnet 55

3.3.6.2 Dauer der stable disease nach Zusatztherapie geordnet $\quad 56$

3.3.7 Verlauf der Tumorgröße 57

3.3.8 Entstehung hepatischer Metastasen

60

3.3.9 Größenverlauf der hepatischen Metastasen 63

3.3.10 Entstehung sonstiger peripherer Metastasen 66

3.3.11 Größenverlauf sonstiger peripherer Metastasen 68

4 Diskussion 72

4.1 Demographie 73

4.1.1 Tumorlokalisation 74

4.1.2 Grading bei Erstdiagnose 74

4.1.3 Staging bei Erstdiagnose 75

4.1.4 Funktionelle / nicht-funktionelle Tumoren 75

4.1.5 Sonstige Therapie 75

4.2 Beobachtungsdauer 76

4.3 Therapie mit SAA 77

4.3.1 Dosierung der SAA

4.3.2 Therapiedauer 81

4.3.3 Verlauf der Symptomatik 81

4.3.4 Verlauf der Tumormarker 84

4.3.5 Stable disease unter SAA 88

4.3.6 Verlauf der Tumorgröße 91

4.3.7 Entstehung hepatischer und sonstiger peripherer Metastasen $\quad 94$

4.3.8 Größenverlauf der hepatischen und sonstigen peripheren Metastasen

4.3.9 Wirksamkeit der SAA im Vergleich zum Zeitraum ohne SAA in detaillierter Gruppenaufteilung 96

4.3.9.1 Verlauf der Tumormarker 97

4.3.9.2 Verlauf der Tumorgröße 98

4.3.9.3 Entstehung hepatischer und sonstiger peripherer Metastasen 
6 Literaturverzeichnis 103

Abbildungsverzeichnis

113

Tabellenverzeichnis 115 


\section{Abkürzungsverzeichnis}

- 5-HIES

- APUD

- Bax

- Bcl-2

- bFGF

- $\mathrm{CCK}$

- CgA

- CLARINET

- NET

- CT

- DOTA

- DOTATATE

- DOTATOC

- DTPA

- EMR

- ENETS

- ERK

- FKBP-12

- G

- GI-NET

- GIST

- IGF

- INF- $\alpha$

- LAG

- M

- MAP-Kinase

- MIB 1-3

- MRT

- mTOR

$-\mathrm{N}$
5-Hydroxyindolessigsäure

amine precursor uptake and decarboxylation

bacl-2-associated X Protein

B-Cel-lymphoma-2

basic fibrinoblast grouth factor

Cholecystokinin

Chromogranin A

Controlled study of Lanreotide antiproliferative response in neuroendocrine Tumors

neuroendokriner Tumor

Computertomographie

1,4,7,10-tetraazacyclododecane-1,4,7,10-tetraacetic acid

(DOTA-Tyr ${ }^{3}$ )-octreotate

(DOTA ${ }^{0}-\underline{P h e}^{1}-$ Tyr $\left.^{3}\right)$-octreotide

diethylene triamine pentaacetic acid

endoskopische Mukosaresektion

European Neuroendocrine Tumor Society

Extrazellulär-regulator-Kinase

FK-binding protein 12

Grading

gastrointestinaler neuroendokriner Tumor

gastrointestinaler Stroma-Tumor

Insulin-like growth factor

Interferon- $\alpha$

long-acting-release

Metastases $=$ Status der Metastasierung (in TNM-Klassifikation)

mitogen-activated-protein-Kinase

murine monoclonal antibodies 1-3

Magnetresonanztomographie

mamalian target of Rapamycin

Nodes $=$ Status der Lymphknoten (in TNM-Klassifikation) 
- Nd:YAG-Laser

- NEC

- NET

- NSE

- PET

- PNET

- PROMID

- RECIST

- SEER

- SHP-1/2

- SRS

- Sst

$-\mathrm{T}$

- TETA

- TNM

- TTHA

- UICC

- VEGF

- VIP

- WHO
Neodym-dotierter Ytrium-Aluminium-Granat-Laser

neuroendokrines Karzinom/neuroendokrine Karzinome

neuroendokriner Tumor/neuroendokrine Tumoren

neuronenspezifische Enolase

Protonen-Emissions-Tomographie

pankreatischer neuroendokriner Tumor/pankreatische neuroendokrine Tumoren

Placebo controlled, double-blind, prospective, Randomized study on the effect of Octreotide LAR in the control of tumor growth in patients with metastatic neuroendocrine MIDgut tu mors

response evaluation criteria in solid tumors

Surveillance, Epidemiology and End-Result Program

Sarcoma-homology-phosphatase-1/2

Somatostatin-Rezeptor-Szintigraphie

Somatostatin-Rezeptoren

Tumor $=$ Status des Primärtumors (in TNM-Klassifikation)

Triethylenetetramine

Tumor, Nodes, Metastases

3,6,9,12-Tetrakis(carboxymethyl)-3,6,9,12-Tetraazatetradecanedioic acid (Triethylenetetraminehexaacetic acid)

Union for International Cancer Control

vascular endothelial growth factor

vasoaktives intestinales Peptid

World Health Organization 


\section{Einleitung}

\subsection{Neuroendokrine Tumoren}

\subsubsection{Definition}

Neuroendokrine Tumoren (NET) stellen eine Gruppe von soliden Malignomen des neuroendokrinen Systems dar. Zu ihnen gehören die so genannten Karzinoide, Gastrinome, Insulinome, Glukagonome, Vipome und Somatosatinome sowie andere pankreatische NET. Ihr Ursprung ist neuroektodermal, selten endodermal. Durch die Produktion hormoneller Peptide können sie - bei funktioneller Aktivität - charakteristische Symptome aufweisen. Eine höhere Prävalenz der NET in der subsaharischen Bevölkerung im Gegensatz zur europäischen lässt eine genetische Prädisposition vermuten (Hauso et al. 2008), die bislang jedoch noch nicht bewiesen werden konnte (Öberg 2010). NET können sporadisch oder in Zusammenhang mit autosomal-dominant vererbbaren Syndromen auftreten (Calender 2000). Zu diesen Syndromen zählen unter anderem: Multiple endokrine Neoplasie Typ I und II, von-Hippel-LindauSyndrom, Neurofibromatose von Recklinghausen und Tuberöse Sklerose. Das „Surveillance, Epidemiology and End-Result Programm“ (SEER) hat gezeigt, dass Inzidenz und Prävalenz dieser Neoplasien in den letzten Jahrzehnten zugenommen haben (Yao et al. 2008), was jedoch auch mit einem steigenden Gesundheitsbewusstsein in der Bevölkerung und einer sich stetig verbessernden Diagnostik zusammenhängen kann.

Bei der Entstehung von NETs können verschiedene Risikofaktoren ausgemacht werden. Diese sind vor allem: Alkohol, Rauchen, weibliches Geschlecht, positive Familienanamnese. Was das Alter der Patienten bei Erstdiagnose betrifft, so können zwei Altersgipfel beobachtet werden: Ein erster zwischen dem 15. bis 25. Lebensjahr und ein zweiter zwischen dem 65. bis 75 . Lebensjahr (Bajetta et al. 2009).

\subsubsection{Klassifikation}

Als Karzinom identifiziert und erstmals beschrieben wurde ein NET 1888 durch Oberndorfer (1907). Die zunächst von Williams und Sandler (1963) erstellte Klassifikation, nach der in Foregut- (Thymus, Ösophagus, Lunge, Magen, Duodenum und Pankreas), Midgut- (Appendix, Ileum, Zökum und Colon ascendens) und Hindgut-Tumoren (distales Kolon und Rektum) unterschieden wurde, hat sich als klinisch und biologisch irrelevant erwiesen. Die heutige Klassifikation beruht auf einer Beschreibung der Lokalisation des Primärtumors, der von ihm sezernierten Hormone und der dadurch verursachten Symptomatik. Nach WHO wird des Wei- 
teren in gut-differenzierte neuroendokrine Neoplasien, mäßig-differenzierte neuroendokrine Neoplasien und schlecht-differenzierte neuroendokrine Karzinome unterschieden, wobei ab dem Stadium der mäßig-differenzierten neuroendokrinen Neoplasie ein erhöhtes Metastasierungsrisiko bestehen kann (Hotz et al. 2011).

Es werden nicht-funktionelle Tumoren, die klinisch stumm verlaufen, von funktionellen Tumoren mit klinischer Manifestation unterschieden. Da jeder NET jedoch Peptide mehrerer Hormone sezerniert, ist auch die Symptomatik der funktionellen NET oft unterschiedlich und unspezifisch.

Nicht-funktionelle NET weisen keine Symptomatik auf und sind daher auch schwer zu diagnostizieren. Oft stellen sich bereits bei Erstdiagnose ein großer Primärtumor und ein fortgeschrittenes Stadium dar. Der Primärtumor befindet sich am häufigsten in Appendix, Duodenum, Kolon oder Rektum. Unspezifische Symptome können Bauchschmerzen, Blutungen, paradoxe Diarrhö sowie B-Symptomatik sein.

Funktionelle NET sind durch die von ihnen produzierten Hormone und dadurch verursachten Symptome charakterisiert. Am häufigsten tritt das so genannte Karzinoid-Syndrom auf. Die vom Primärtumor produzierten Hormone werden in der Leber verstoffwechselt, weswegen sie keine oder nur geringe Symptome hervorrufen. Nach Metastasierung in die Leber jedoch kommt es bei $40 \%$ der Patienten mit NET nicht-pankreatischen Ursprungs zu spezifischen Symptomen (Fothner und Weber 2012), die auf die Sekretion vasoaktiver Stoffe wie Serotonin und Tachykinine zurückzuführen sind (Creutzfeldt 1996): Flush (63 \%-94 \%), Diarrhö (68 \%-84\%), Bauchschmerzen (10\%-55\%), Teleangiektasien (25\%), Bronchokonstriktion (3\%-19 \%) und Ödeme (19\%) (Rubin et al. 1999; Schnirer et al. 2003; Kaltsas et al. 2004). Die damit verbundene Karzinoid-Krise, die durch einen Überschuss an vasoaktiven Stoffen entsteht, ist durch Hypertonus, Arrhythmie, Lungenobstruktion und Delir gekennzeichnet (Turaga und Kvols 2011). Sie kann spontan auftreten, steht jedoch meist im Zusammenhang mit Stress, Narkose, OP oder Chemotherapie und stellt laut Schnirer et al. (2003) die häufigste Komplikation mit erheblicher Einschränkung der Lebensqualität dar. Das Karzinoid-Syndrom kann so unbehandelt durch das ständige Einwirken vasoaktiver Stoffe auf den Organismus zu Karzinoid-Herzschäden führen. Diese stellen sich als fibröse Plaques im Endokard vor allem des rechten Herzens und der Trikuspidal- und Pulmonalklappe dar, was zu einer Rechtsherzinsuffizienz führen kann. Diese rechtsventrikuläre Insuffizienz durch Myokardfibrose bei Karzinoid wird auch Hedinger-Syndrom genannt. Ihr Auftreten ist mit einer erhöhten Morbidität und Mortalität vergesellschaftet. 10-20 \% der Patienten mit Karzinoid-Syndrom weisen 
bereits bei Erstdiagnose einen durch diese Symptomatik bedingten Herzschaden auf (Modlin et al. 2008).

Pankreatische NET(PNET) stellen eine gesonderte Gruppe innerhalb der NET dar. Sie werden durch das Wirkungsprofil der von ihnen sezernierten Hormone symptomatisch. So kann es durch ein Gastrinom beispielsweise zu einem Zollinger-Ellison-Syndrom mit peptischen Ulcera, Diarrhö und Bauchschmerzen kommen. Ein Glukagonom führt zu Hyperglykämie und ist mit Diabetes mellitus, Thrombosen und Anämie vergesellschaftet. Als Kardinalsymptom ist hier das Erythema necrolyticum migrans zu nennen, welches in 60-90\% der Fälle auftritt (Wermers et al. 1996). Insulinome führen zu Hypoglykämie und ein Vipom beziehungsweise das Verner-Morrison-Syndrom kann durch Diarrhö, Hypokaliämie und metabolischer Azidose klinisch manifest werden (Öberg 2010).

Die Einteilung in funktionelle und nicht-funktionelle NET innerhalb der PNETs ist leider sehr heterogen. Als nicht-funktionell werden generell PNETs benannt, die beim Patienten keine Symptome durch Hormonüberschuss verursachen. Trotzdem können diese Patienten erhöhte Hormonspiegel aufwiesen. Diese also rein klinische Unterteilung führt zu uneinheitlichen Klassifikationen. Daher differieren auch die Zahlen der Anteile nicht-funktioneller PNET am Gesamtanteil der PNET, der mit 20-60 \% beschrieben wird (Halfdanarson et al. 2008).

\subsubsection{Grading}

Das Grading basiert auf der Anzahl der Mitosen pro Quadratmillimeter und dem Ki-67-Index (vgl. ENETS Consensus Guidelines for the Diagnosis and Treatement of Neuroendocrine Tumors, 2012). Ki-67 ist ein Antigen, das in jeder Phase der Mitose auf der Chromosomenoberfläche nachgewiesen werden kann. In der G0-Phase des Zellzyklus ist es hingegen nicht nachweisbar, da in dieser Phase keine Zellteilung stattfindet. Somit kann Ki-67 als Marker für die Zellteilung und damit zur Bestimmung der Wachstumsaktivität einer Zellpopulation eingesetzt werden (vgl. auch Beschreibung der Methodik, Kapitel 2.1.1).

Tabelle 1 gibt eine Übersicht über das Grading anhand der Ki-67-Werte und Mitoseraten.

Tabelle 1: Grading von NET nach WHO-Klassifikation (nach Fottner und Weber 2012).

\begin{tabular}{|l|l|l|}
\hline Grad & Ki-Index (in \%) & Mitosen $\left(\right.$ pro $\left.2 \mathbf{~ m m}^{\mathbf{2}}\right)$ \\
\hline G1 & $\leq 2$ & $<2$ \\
\hline G2 & $3-20$ & $2-20$ \\
\hline G3 & $>20$ & $>20$ \\
\hline
\end{tabular}

Dabei entsprechen:

- gut-differenzierte neuroendokrine Neoplasien (gut-differenzierte NET) 
$=\mathrm{G} 1=\mathrm{WHO} 1$, auch bezeichnet als niedrig-proliferative neuroendokrine Neoplasien

- mäßig-differenzierte neuroendokrine Neoplasien (mäßig-differenzierte NET)

$=\mathrm{G} 2=\mathrm{WHO} 2$, auch bezeichnet als mäßig-proliferative neuroendokrine Neoplasien

- schlecht-differenzierte neuroendokrine Karzinome (schlecht-differenzierte NEC)

$=\mathrm{G} 3=\mathrm{WHO}$ 3, auch bezeichnet als hoch-proliferative neuroendokrine Neoplasien

\subsubsection{Staging}

Rindi et al. (2006, 2007) erstellten eine TNM-Klassifikation für GI-NET, die von Sobin et al. (2009) überarbeitet und aktualisiert wurde. Die Klassifizierung richtet sich dabei nach den vom Tumor infiltrierten Schichten der Darmwand und dem Ausmaß der Metastasierung. Die meisten NETs sind maligne und metastasieren vorwiegend in regionale Lymphknoten und in die Leber, seltener in Knochen, Haut und Gehirn.

Für mäßig-differenzierte NETs und schlecht-differenzierte NECs ergibt sich folgende Klassifikation für den Status T des Primärtumors, welche in Tabelle 2-6 dargestellt ist:

\section{Magen:}

Tabelle 2: T-Klassifikation von NET des Magens (Sobin et al. 2009)

\begin{tabular}{|l|l|}
\hline T1 & $\begin{array}{l}\text { Tumoren, die auf die Mukosa begrenzt sind und zwischen 0,5 mm und } 1 \mathrm{~cm} \text { messen } \\
\text { oder bereits in die Submukosa eindringen, aber an der Stelle ihres größten Durch- } \\
\text { messers nicht größer als } 1 \mathrm{~cm} \text { sind }\end{array}$ \\
\hline T2 & $\begin{array}{l}\text { Tumoren, die bis in die Muscularis propria eindringen oder an der Stelle ihres größ- } \\
\text { ten Durchmessers größer als } 1 \mathrm{~cm} \text { sind }\end{array}$ \\
\hline T3 & $\begin{array}{l}\text { Tumoren, die bis in die Subserosa eindringen } \\
\text { ne und Strukturen hineinwachsen }\end{array}$ \\
\hline T4 & \multicolumn{2}{|l}{} \\
\hline
\end{tabular}

Dünndarm:

Tabelle 3: T-Klassifikation von NET des Dünndarms (Sobin et al. 2009)

\begin{tabular}{|l|l|}
\hline T1 & $\begin{array}{l}\text { Tumoren, die in die Lamina propria oder Submukosa einwachsen, aber an der Stelle } \\
\text { ihres größten Durchmessers nicht größer als } 1 \mathrm{~cm} \text { sind }\end{array}$ \\
\hline T2 & $\begin{array}{l}\text { Tumoren, die in die Muscularis propria einwachsen oder an der Stelle ihres größten } \\
\text { Durchmessers größer als } 1 \mathrm{~cm} \text { sind }\end{array}$ \\
\hline T3 & $\begin{array}{l}\text { Tumoren des Jejunums und Ileums, die in die Subserosa einwachsen. Außerdem } \\
\text { Tumore der Ampulle und des Duodenums, die in das Pankreas oder Peritoneum }\end{array}$ \\
\hline
\end{tabular}




\begin{tabular}{|l|l|}
\hline & einwachsen \\
\hline T4 & $\begin{array}{l}\text { Tumoren, die die Serosa bzw. das Peritoneum penetrieren oder in umliegende Orga- } \\
\text { ne und Strukturen hineinwachsen }\end{array}$ \\
\hline
\end{tabular}

Dickdarm:

Tabelle 4: T-Klassifikation von NET des Dickdarms (Sobin et al. 2009)

\begin{tabular}{|l|l|}
\hline T1a & $\begin{array}{l}\text { Tumoren, die die Lamina propria oder die Submukosa infiltrieren und an der Stelle } \\
\text { ihres größten Durchmessers kleiner als } 1 \mathrm{~cm} \text { sind }\end{array}$ \\
\hline T1b & $\begin{array}{l}\text { Tumoren, die die Lamina propria oder die Submukosa infiltrieren und an der Stelle } \\
\text { ihres größten Durchmessers zwischen } 1 \mathrm{~cm} \text { und } 2 \mathrm{~cm} \text { groß sind }\end{array}$ \\
\hline T2 & $\begin{array}{l}\text { Tumoren, die in die Muscularis propria einwachsen oder an der Stelle ihres größten } \\
\text { Durchmessers größer als } 2 \mathrm{~cm} \text { sind }\end{array}$ \\
\hline T3 & $\begin{array}{l}\text { Tumoren, die in die Subserosa oder in nicht-peritonealisiertes Gewebe, welches das } \\
\text { Kolon und Rektum umgibt, einwachsen. }\end{array}$ \\
\hline T4 & $\begin{array}{l}\text { Tumoren, die das Peritoneum penetrieren oder in umliegende Organe und Strukturen } \\
\text { hineinwachsen }\end{array}$ \\
\hline
\end{tabular}

Appendix:

Tabelle 5: T-Klassifikation von NET der Appendix (Sobin et al. 2009)

\begin{tabular}{|l|l|}
\hline T1a & Tumoren, die an der Stelle ihres größten Durchmessers höchstens $1 \mathrm{~cm}$ messen \\
\hline T1b & $\begin{array}{l}\text { Tumoren, die an der Stelle ihres größten Durchmessers mehr als } 1 \mathrm{~cm} \text { aber höchs- } \\
\text { tens } 2 \mathrm{~cm} \text { messen }\end{array}$ \\
\hline T2 & $\begin{array}{l}\text { Tumoren, die an der Stelle ihres größten Durchmessers mehr als } 2 \mathrm{~cm} \text { aber höchs- } \\
\text { tens } 4 \mathrm{~cm} \text { messen. Außerdem Tumore, die sich bereits bis ins Zökum ausdehnen }\end{array}$ \\
\hline T3 & $\begin{array}{l}\text { Tumoren, die an der Stelle ihres größten Durchmessers mehr als } 4 \mathrm{~cm} \text { messen. Au- } \\
\text { ßerdem Tumore, die sich bereits bis ins Ileum ausdehnen }\end{array}$ \\
\hline T4 & $\begin{array}{l}\text { Tumoren, die bereits das Peritoneum oder andere umliegende Organe und Strukturen } \\
\text { penetrieren }\end{array}$ \\
\hline
\end{tabular}

Pankreas:

Tabelle 6: T-Klassifikation von NET des Pankreas (Sobin et al. 2009)

\begin{tabular}{|l|l|}
\hline T1 & $\begin{array}{l}\text { Tumoren, die auf das Pankreas beschränkt und an der Stelle ihres größten Durch- } \\
\text { messers nicht größer als } 2 \mathrm{~cm} \text { sind }\end{array}$ \\
\hline T2 & $\begin{array}{l}\text { Tumoren, die auf das Pankreas beschränkt und an der Stelle ihres größten Durch- } \\
\text { messers zwischen } 2 \mathrm{~cm} \text { und } 4 \mathrm{~cm} \text { groß sind }\end{array}$ \\
\hline
\end{tabular}




\begin{tabular}{|l|l|}
\hline T3 & $\begin{array}{l}\text { Tumoren, die auf das Pankreas beschränkt und an der Stelle ihres größten Durch- } \\
\text { messers größer als } 4 \mathrm{~cm} \text { sind oder Tumore, die bereits in Duodenum oder in die Gal- } \\
\text { lenblase einwachsen }\end{array}$ \\
\hline T4 & $\begin{array}{l}\text { Tumoren, die in andere Organe (Magen, Milz, Kolon, Nebenniere) oder in die Wand } \\
\text { großer Gefäße (Truncus coeliacus, Arteria mesenterica superior) einwachsen }\end{array}$ \\
\hline
\end{tabular}

Nach der TNM-Klassifikation wird zudem der Lymphknotenstatus $N$ und der Status $M$ der Fernmetastasen angegeben. Diese sind in Tabelle 7 und 8 aufgeführt. Die sich daraus ergebende Klassifikation der Tumorstadien ist in Tabelle 9 dargestellt.

Tabelle 7: N-Klassifikation von NET (Sobin et al. 2009)

\begin{tabular}{|l|l|}
\hline Nx & regionale Lymphknoten können nicht beurteilt werden \\
\hline N0 & keine Metastasen in regionalen Lymphknoten \\
\hline N1 & Metastase in regionalen Lymphknoten nachweisbar \\
\hline
\end{tabular}

Tabelle 8: M-Klassifikation von NET (Sobin et al. 2009)

\begin{tabular}{|l|l|}
\hline Mx & keine Aussage über Fernmetastasen möglich \\
\hline M0 & keine Fernmetastasen \\
\hline M1 & Fernmetastasen nachgewiesen \\
\hline
\end{tabular}

Für die allgemeinen Tumorstadien ergibt sich damit folgende Einteilung:

Tabelle 9: Tumorstadien von NET (Sobin et al. 2009)

\begin{tabular}{|l|l|l|l|}
\hline I & T1 & N0 & M0 \\
\hline II A & T2 & N0 & M0 \\
\hline II B & T3 & N0 & M0 \\
\hline III A & T4 & N0 & M0 \\
\hline III B & jedes T & N1 & M0 \\
\hline IV & jedes T & jedes N & M1 \\
\hline
\end{tabular}

Die hier aufgeführte TNM-Klassifikation ist in weiten Teilen deckungsgleich mit der ENETSKlassifikation (vgl. ENETS 2012). Abweichungen zwischen den beiden Klassifikationen finden sich bei PNET sowie NET der Appendix und bei den schlecht-differenzierten NEC. Für diese Tumoren empfiehlt sich eine Angabe beider Klassifikationen.

Grading und Staging haben auch eine prognostische Bedeutung. Als prognostisch ungünstige Faktoren gelten: Tumorgröße $>2 \mathrm{~cm}$, Mitoserate $>20 \%$ bzw. Ki-67-Index $>20 \%$ (= G3- 
Tumor), Infiltration in Gefäße, ausgeprägte lokale Infiltration und vorhandene Metastasen (Cameron und Ramadori 2010).

\subsection{Diagnostik}

Eine multimodale Diagnostik ist unbedingt erforderlich. Dazu zählen klinische Symptomatik, Histopathologie, Biomarker, Hormonspiegel und Bildgebung einschließlich spezieller Verfahren wie Somatostatin-Rezeptor-Szintigraphie und PET mit radioaktiv markierten Somatostatin-Analoga.

\subsubsection{Klinische Symptomatik}

In Ermangelung einer frühen symptomal spezifischen Manifestation können bei bis zu $50 \%$ der Patienten bei Erstdiagnose schon Metastasen nachgewiesen werden (Yao et al. 2008). Die ersten Metastasen sind in den regionalen Lymphknoten zu finden. Später kann auch eine Tumorlast in der Leber nachgewiesen werden. In sehr fortgeschrittenen Stadien kommt es auch zu einer Metastasierung in weiter entfernten Organen, vor allem in den Knochen. Die für das Karzinoid-Syndrom charakteristischen Symptome treten bei funktionellen NET mit vorhandenen Lebermetastasen auf (Metz und Jensen 2008).

\subsubsection{Biomarker}

Serotonin (5-Hydroxytrypamin, 5-HT) ist ein biogenes Amin, welches von den meisten Arten von NETs gebildet wird. Es wird physiologische unter anderem in den EC-Zellen des Darms aus der Aminosäure Tryptophan gebildet und weist sowohl Eigenschaften eines Hormons als auch eines Neurotransmitters auf. Der Serotoninspiegel kann im Blut bestimmt werden und wurde in früheren Jahren als Parameter bei der Verlaufskontrolle solcher Tumorerkrankungen eingesetzt, hat nach neueren Studien jedoch keine Bedeutung für Diagnose und Follow-up (O'Toole et al. 2009).

5-Hydroxyindolessigsäure (5-HIES) ist ein Serotoninmetabolit, der bei bestimmten Typen funktioneller NETs mit Karzinoidsymptomatik im 24-Stunden-Sammelurin mittels elektrochemischer Detektion nachgewiesen werden kann. In metastasierten funktionellen Tumoren, v.a. bei vorhandenem Karzinoid-Syndrom spiegelt der 5-HIES-Wert das Tumorvolumen wider. Bei nicht-funktionellen NET und bei M0-Status ist der Wert allerdings negativ (O'Toole et al. 2009). Falsch positive beziehungsweise falsch-negative Werte ergeben sich vor allem nach dem Genuss tryptophanhaltiger Nahrungsmittel, worunter unter anderem Vollkornprodukte, Naturreis, Sojabohnen, Käse, Quark und Nüsse fallen (Feldman 1986; Kema et al. 1992). Auch können bestimmte Medikamente den 5-HIES-Spiegel verändern. Beispielsweise 
wird der Abbau von Serotonin zu 5-HIES durch Hemmer der Monoaminooxidase, die vor allem bei Parkinsonpatienten und als Antidepressiva eingesetzt werden, verlangsamt, was zu einem falschniedrigen Wert führt. Auch Serotonin-Antagonisten führen durch die verminderte Ausschüttung an Serotonin zu einer verminderten Konzentration an 5-HIES im Urin. Bei einer Verlaufskontrolle des 5-HIES unter Serotonin-Antagonisten sind diese also rechtzeitig vorher abzusetzen um nicht einen falsch-niedrigen Wert zu erhalten. Bei Niereninsuffizienz und dialysepflichtigen Patienten ergibt sich außerdem ein falsch-niedriger Wert, da hier die Ausscheidung des 5-HIES durch das Grundleiden vermindert ist. Bei Malabsorbtionssyndrom sind die Tryptophanmetabolite, zu denen auch 5-HIES zählt, im Urin erhöht, was zu falschhohen Ergebnissen führen kann. Der genaue Mechanismus dahinter ist nicht bekannt. Die Erhöhung der 5-HIES-Werte im Urin wurde jedoch schon von Challacombe et al. (1972) bei Kindern mit Zöliakie beobachtet. Eine Beobachtung der 5-HIES-Werte bei Kindern mit Kwashiorkor durch Teotia und Teotia (1975) lässt einen Zusammenhang zwischen diesen Werten und dem Auftreten der Steatorrhö vermuten. Patienten, die wegen eines GI-NETs operiert wurden, leiden häufig unter Malabsorption oder/und Steatorrhö, weswegen diese Zusammenhänge im Hinblick auf die Bestimmung der 5-HIES-Werte stets beachtet werden sollten.

Erhöhte Werte von Neuronspezifischer Enolase (NSE) werden öfter bei mäßig-differenzierten NET bzw. schlecht-differenzierten NEC nachgewiesen. NSE kann im Serum bestimmt werden. Sie ist ein Enzym des Glukosestoffwechsels, deren Isomere in Nervenzellen des zentralen und peripheren Nervensystems und in neuroendokrinem Gewebe vorkommen. Im neuroendokrinen Gewebe finden sich unter anderem so genannte APUD-Zellen, in denen vor allem das $\gamma / \gamma$-Dimer der NSE vorhanden ist. APUD steht für amine precursor uptake and decarboxylation und beschreibt einen biochemischen Vorgang bei der Bildung von MonoaminNeurotransmittern wie Serotonin, Dopamin und Histamin, der in Zellen neuroendokrinen Gewebes stattfindet. Eine erhöhte NSE lässt auf eine erhöhte Anzahl von zugrunde gehenden APUD-Zellen schließen (Bajetta et al. 1999).

Chromogranin A $(\mathrm{CgA})$ ist ein saures Glykoprotein und fester Bestandteil der Membran von Chromaffingranula der neuroendokrinen Zellen. Sowohl bei funktionellen, als auch bei nichtfunktionellen Tumoren kann CgA mittels Blutprobe (Serum- oder Plasmaanalyse) oder direkt in einer Gewebeprobe per Immunhistochemie nachgewiesen werden (Rindi und Klöppel 2004). Die Menge des nachgewiesenen CgA korreliert mit der Tumorlast (Pirker et al. 1998) und soll auch die Wachstumsaktivität des Tumors widerspiegeln (Janson et al. 1997). Außerdem nimmt der CgA-Wert im metastasierten Stadium zu. CgA ist zudem sensitiver als andere 
Biomarker (Bajetta et al. 1999; Korse et al. 2009) und stellt somit das diagnostische Verfahren erster Wahl dar. Allerdings wird der CgA-Wert durch verschiedene Faktoren beeinflusst, die zu falsch-positiven Ergebnissen führen können. Hierzu zählen eine eingeschränkte Nierenfunktion, Parkinsonkrankheit, ein unbehandelter arterieller Hypertonus, Schwangerschaft, Steroidtherapie, Typ-A-Gastritis und eine Therapie mit Protonenpumpenhemmern oder $\mathrm{H}_{2}$ Blockern. Bei einer Niereninsuffizienz kann der Wert sogar bis $2000 \mu \mathrm{g} / \mathrm{l}$ erhöht sein (Norm: $<100 \mu \mathrm{g} / \mathrm{l}$ im Serum bzw. < $150 \mu \mathrm{g} / \mathrm{l}$ im Plasma) (Degorce et al. 1999).

Tabelle 10 stellt die verschiedenen Biomarker und ihre Eigenschaften zusammengefasst dar.

Tabelle 10: Eigenschaften der verschiedenen Biomarker

\begin{tabular}{|c|c|c|c|c|c|}
\hline Biomarker & Bedeutung/Vorkommen & Nachweis in & Testverfahren & Korrelation mit & falsch-positiv bzw. falsch-negativ bei \\
\hline Serotonin & $\begin{array}{l}\text { Von neuroendokrinen } \\
\text { Zellen gebildeter } \\
\text { Neurotransmitter }\end{array}$ & $\begin{array}{l}\text { beinahe alle Arten von } \\
\text { NET }\end{array}$ & Blutanalyse & $\begin{array}{l}\text { sehr unspezifisch, } \\
\text { eigentlich obsolet }\end{array}$ & \\
\hline 5-HIES & Serotoninmetabolit & $\begin{array}{l}\text { funktionelle NETs mit } \\
\text { Karzinoidsymptomatik }\end{array}$ & $\begin{array}{l}\text { Analyse des } \\
24 \mathrm{~h} \text {-Sammelurin }\end{array}$ & $\begin{array}{l}\text { Tumorvolumen bei } \\
\text { metastasierten NETs }\end{array}$ & \begin{tabular}{|l} 
- tryptophanhaltige Nahrung, \\
Malabsorbtionssyndrom \\
- Niereninsuffizienz und \\
dialysepflichtige Patienten \\
- bestimmte Medikamente \\
\end{tabular} \\
\hline NSE & $\begin{array}{l}\text { Enzym des } \\
\text { Glukosestoffwechsels } \\
\text { neuroendokriner Zellen }\end{array}$ & $\begin{array}{l}\text { mäßig-differenzierte } \\
\text { NETs bzw. schlecht- } \\
\text { differenzierte NECs }\end{array}$ & Serumanalyse & $\begin{array}{l}\text { Anzahl der APUD- } \\
\text { Zellen }\end{array}$ & \\
\hline $\mathrm{CgA}$ & $\begin{array}{l}\text { Saures Glykoprotein, } \\
\text { fester Bestandteil der } \\
\text { Membran von } \\
\text { Chromaffingranula } \\
\text { neuroendokriner Zellen }\end{array}$ & $\begin{array}{l}\text { - funktionelle NETs } \\
\text { - nicht-funktionelle } \\
\text { NETs }\end{array}$ & $\begin{array}{l}\text { - Serum-/ } \\
\text { Plasmaanalyse } \\
\text { - Gewebeprobe }\end{array}$ & $\begin{array}{l}\text { - Tumorlast } \\
\text { - Wachstumsaktivität } \\
\text { - Erhöhung bei } \\
\text { Metastasierung } \\
\text { sensitivster Marker }\end{array}$ & $\begin{array}{l}\text { - eingeschränkte Nierenfunktion/ } \\
\text { Niereninsuffizienz } \\
\text { - Morbus Parkinson } \\
\text { - unbehandelter arterieller } \\
\text { Hypertonus } \\
\text { - Schwangerschaft } \\
\text { - Steroidtherapie } \\
\text { - Typ-A-Gastritis } \\
\text { - Therapie mit PPI oder } \mathrm{H}_{2} \text {-Blockern }\end{array}$ \\
\hline
\end{tabular}

Die Bestimmung von bestimmten Hormonspiegeln wie Gastrin, Glukagon, Insulin/Proinsulin und VIP kann vor allem bei PNETs von Bedeutung sein. So kann zum Beispiel bei Verdacht auf ein Insulinom ein 72-Stunden-Fasten-Test durchgeführt werden. Analog dazu wird bei Verdacht auf ein Gastrinom ein Sekretin-Test durchgeführt. Hierbei ist zu beachten, dass falsch-positive Ergebnisse durch eine Gastritis oder langjährige Einnahme von Protonenpumpenhemmern verursacht werden können (O’Toole et al. 2009).

\subsubsection{Endoskopie}

In Regionen, die der Gastroskopie oder Koloskopie zugänglich sind, lassen sich NETs mit dieser Methode sehr gut detektieren. Eine gleichzeitig gewonnene Gewebeprobe kann zur histologischen Sicherung der Diagnose dienen. Zur ersten Abschätzung der Infiltrationstiefe eignet sich außerdem die Endosonographie (Riemann et al. 2010). Die Endosonographie kann schon während der endoskopischen Untersuchung einen wesentlichen Beitrag zu Diagnostik und Staging des NET beitragen (Scherübel et al. 2010). Turaga und Kvols (2011) beschreiben 
eine Rate von 45-60 \% der duodenalen Läsionen und 90-100 \% der pankreatischen Läsionen, die durch eine Endosonographie detektiert werden können. Eine endgültige und damit auch prognostisch und therapeutisch relevante Aussage über die Infiltrationstiefe kann jedoch nur mittels Histopathologie getroffen werden.

\subsubsection{Radiologische und nuklearmedizinische Untersuchung}

Nuklearmedizinische und radiologische Bildgebung spielen vor allem bei der Lokalisation des Primärtumors und beim Tumor-Staging eine Rolle. Standartverfahren hierbei sind $C T$ und $M R T$. Doch auch die Ultraschall-Sonographie hat vor allem bei NETs des Duodenums und des Pankreas eine große Bedeutung (Öberg und Castellano 2011).

Als bildgebende Untersuchung erster Wahl gilt die Ganzkörperdarstellung mittels Somatostatin-Rezeptor-Szintigraphie (SRS) (Usmani et al. 2011). Am häufigsten eingesetzt wird Indium-111-Pentereotid, das eine hohe Affinität für den Somatostatin-Rezeptor sst $_{2}$, den von NETs am häufigsten exprimierten Rezeptoren, hat (Reubi et al. 2001). Ricke et al. (2001) postulierten, dass mittels SRS Primärtumoren und Metastasen auch in frühem Stadium mit einer Sensitivität von 80-90 \% detektiert werden können und dass die SRS bezüglich des Stagings und der Verlaufskontrolle bei NETs den anderen bildgebenden Verfahren überlegen sei. So stellt sie auch die sensitivste Single-Screening-Methode bei extrahepatisch manifesten Tumoren dar (Falconi et al. 2006). Durch die SRS können bereits kleine Läsionen, die klinisch noch nicht suspekt wirken, detektiert werden, was das Staging optimiert. Außerdem kann diese Untersuchung eine Entscheidungshilfe hinsichtlich einer Therapie liefern: Fehlende Anreicherung an der Lokalisation eines bekannten Tumors stellen einen ungünstigen prognostischen Faktor dar, da eine Therapie mit SAA bei nur geringfügiger Dichte oder Fehlen von Somatostatin-Rezeptoren wenig erfolgsversprechend ist. Gleichzeitig jedoch kann bei einer hohen Rezeptordichte ein Ansprechen auf eine Therapie mit SAA erwartet werden. Des Weiteren können Patienten, die für eine Therapie mit radioaktiv markierten SAA in Betracht kommen, durch diese Methode identifiziert und deren Verlauf kontrolliert werden (Usmani et al. 2011). Vorsicht sollte bei einer vorher bestehenden Therapie mit SAA geboten sein, da diese das Signal von $\left[{ }^{111}\right.$ In-DTPA $\left.{ }^{0}\right]$-Octreotid maskieren können. Eine solche Therapie ist daher rechtzeitig vor der Untersuchung zu pausieren.

In letzter Zeit erlangte die PET mit Gallium-68-DOTATOC immer größere Bedeutung. Gallium-68-DOTATOC ist ein Komplex aus [Ga-68]Ga(III) und einem Peptid, dass Somatostatin ähnelt. Es besteht aus zwei Komponenten, die durch kovalente Bindungen chemisch miteinander konjugiert sind: Erstens dem Komplexbildner 1,4,7,10-Tetraazacyclododecan1,4,7,10-Tetraessigsäure, genannt DOTA, und zweitens dem Octreotid-Analogon D-Phe- 
cyclo[Cys-Try-D-Trp-Lys-Thr-Cys]-Thr(ol), genannt TOC. Dieses Peptid besitzt gegenüber Somatostatin-Rezeptoren ähnliche Bindungseigenschaften wie Somatostatin selbst. Durch die Bindung an den Rezeptor kann Gallium-68-DOTATOC somit zur Bildgebung der Rezeptorexpression verwendet werden. Dadurch kann nicht nur eine Aussage über die Tumorlokalisation, sondern auch über die Rezeptordichte getroffen werden. Diese Methode ist sensitiver als die SRS und soll diese in naher Zukunft ersetzen (Öberg 2010).

Diskutiert werden ebenso genetische Veränderungen in neuroendokrinen Tumorzellen. Durch Entwicklung von Genmarkern für die Gene, die für die Entartung verantwortlich und pathognomonisch sind, könnten neue Möglichkeiten der Detektion von NET entstehen (Duerr et al. 2008; Leja et al. 2009).

Neue diagnostische Werkzeuge wie Gallium-68-DOTATOC-PET, Bio- und Genmarker lassen auf eine frühere Erkennung der Tumoren und eine bessere prognostische Aussage hinsichtlich des Therapieerfolgs hoffen.

\subsection{Therapie}

Allgemeine Therapieziele stellen die vollständige Tumorresektion und im Falle nichtresezierbarer Tumoren die Kontrolle von Tumorsymptomatik und Tumorwachstum dar. Neben der chirurgischen Therapie sind auch Biotherapie, Chemotherapie und Therapie mit radioaktiven Stoffen von großer Bedeutung.

Ein von Öberg (2010) erstelltes Therapieschema sieht folgende Behandlung bei metastasierten NETs vor: G1- und G2-Tumoren können nach einer chirurgischen Tumorresektion durch eine alleinige Biotherapie mit SAA behandelt werden. Schlecht-differenzierte NECs werden radikal onkologisch operiert. Es folgt eine chemotherapeutische Behandlung mit zusätzlicher Gabe von SAA zur Symptomkontrolle. Eventuell vorhandene Lebermetastasen werden nach der jeweils geeigneten Methode angegangen. Bei Tumoren, die primär nicht-resezierbar erscheinen, jedoch in der Staginguntersuchung noch keine Fernmetastasen nachweisen, kann auch der Ansatz einer neoadjuvanten Chemotherapie mit dem Ziel des Downstagings und nachfolgender kurativer Operation gewählt werden. Zusätzlich kann unabhängig vom Tumorstadium eine Radiotherapie mit markierten SAA wie $\mathrm{Lu}^{177}$ DOTA-Octreotid oder $\mathrm{Y}^{90}$ DOTATOC eingesetzt werden. Nicht-metastasierte NETs können häufig durch eine alleinige operative Therapie kuriert werden.

Der Erfolg einer Therapie kann hauptsächlich durch drei Kriterien beschrieben werden: Verlauf der Tumorgröße, Ansprechen der Tumormarker und Ausmaß der Beschwerdelinderung. 
Hierbei stellen der Verlauf der Tumorgröße und damit die Dauer der stable disease den wichtigsten Parameter dar.

\subsubsection{Chirurgische Therapie}

Die chirurgische Resektion stellt nach wie vor den Goldstandard und zugleich die einzige Möglichkeit der kurativen Therapie dar (Pavel et al. 2012). Sie umfasst die operative Resektion des Primärtumors und Entfernung aller regionalen Lymphknoten. Die Wahl der Operationsmethode hängt wesentlich von der Tumorgröße und dem Stadium ab: G1-Tumoren mit einer Größe $<10$ mm können, da sie ein niedriges hämatologisches und lymphatisches Metastasierungsrisiko haben, durch eine endoskopische Mukosaresektion (EMR) oder eine lokalablative Abtragung entfernt werden sofern sie einem endoskopischen Eingriff zugängig sind. Dies trifft vor allem auf NETs des Duodenums und des Rektums zu. Größere und aggressivere Tumoren werden hingegen radikal-onkologisch operiert (Scherübl et al. 2010).

Wie in Kapitel 1.2.1 schon erwähnt, befinden sich bis zu $50 \%$ der Patienten bei Erstdiagnose schon in einem metastasierten Stadium, in dem eine komplette Resektion nicht mehr möglich ist. In diesem Fall kann eine palliative zytoreduktive Operation im Sinne eines Debulkings durchgeführt werden. Die Notwenigkeit einer solchen Therapie ist in der Korrelation von Tumormasse zur Symptomatik begründet: Eine Reduktion der Tumormasse um mindestens $90 \%$ kann bei $60 \%$ der Patienten zur Beseitigung der Karzinoidsymptomatik führen (Öberg 2010). Dadurch kann auch in metastasiertem Stadium die Prognose verbessert werden (Plöckinger et al. 2004).

Die Problematik des Karzinoid-Syndroms stellt sich meist erst bei vorhandenen Lebermetastasen. Zur Symptomkontrolle eines metastasierten NET ist also auch die Therapie des Leberbefalls von großer Bedeutung. Die hierfür eingesetzten Methoden sind: Operative Resektion, Leberarterien-Embolisation der -Ligation, Metastasen-Ablation, Laserinduzierte Thermotherapie, Radiofrequenzablation und Lebertransplantation:

Neben einer chirurgischen Resektion werden vor allem bei ausgeprägtem Befall der Leber auch die Leberarterien-Embolisation oder-Ligation angewandt. Bei einer Embolisation wird oft Ethanol eingesetzt, radioaktive Stoffe wie ${ }^{90} \mathrm{Y}$ und Zytostatika wie Doxorubicin oder Cisplatin finden hier jedoch auch ihre Anwendung. Der Erfolg dieser Methode ist in der Literatur mehrfach belegt: Desai et al. (2001) beschrieben in 59-100 \% der Fälle eine Reduktion der Symptome durch eine Embolisation. Ein Rückgang des Biomarkers 5-HIES wurde von Roche et al. (2003) bei 51-91\% der so behandelten Patienten geschildert.

Die Metastasen-Ablation wird nur bei nicht-resezierbaren Metastasen mit einem Durchmesser von bis zu 5-7 cm durchgeführt (Strosberg et al. 2011 a). Grundsätzlich stehen zwei Metho- 
den zur Verfügung: Die Kryoablation und die Radiofrequenzablation, wobei letztere bis zu einem Metastasendurchmesser von $4 \mathrm{~cm}$ in Betracht zu ziehen ist (Bajetta et al. 2009).

Eine weitere Möglichkeit der minimalinvasiven Therapie von Lebermetastasen stellt die Laserinduzierte Thermotherapie dar (Pavel et al. 2012). Hierbei wird ein Nd:YAG-Laser direkt im Gewebe platziert. Die Wellenlänge dieses Lasers befindet sich im Infrarotbereich, was zur Folge hat, das es von gut durchblutetem Gewebe, wie den Metastasen, gut absorbiert wird, von Wasser jedoch weniger. Durch das auf den Tumor gegebene Laserlicht kommt es zur thermischen Zerstörung der Tumorzellen unter Schonung des umliegenden Gewebes.

Vor allem bei jüngeren Patienten findet bei ausgeprägtem Befall und schwerer Karzinoidsymptomatik in letzter Zeit auch die Lebertransplantation immer mehr Anwendung. Wobei die einzige Indikation dazu momentan ein nicht-resezierbarer Tumor oder die palliative Symptomkontrolle darstellen (Fan et al. 2015).

\subsubsection{Biotherapie mit SSA}

Den wichtigsten Pfeiler der medikamentösen Therapie stellen die SAA dar.

Somatostatin, ein kleines cyclisches Peptid, ist ein endokriner Inhibitor der Freisetzung verschiedener Hormone. $\mathrm{Zu}$ ihnen zählen Serotonin, Insulin, Glukagon, Gastrin und einige Wachstumsfaktoren wie IGF-1 (insulin-like growth factor 1) (Grozinsky-Glasberg et al. 2008). Somit inhibiert Somatostatin die Magensäureproduktion, die exokrine Sekretion des Pankreas, die Nahrungsmittelabsorption im Darm, die Sekretion von Galle und die Entleerung der Gallenblase und der Speicheldrüsen. Außerdem führt es zu einer Reduktion der MagenDarm-Motilität und des Blutflusses im Splanchnikus- und Pfortadergebiet. Somatostatin wird vor allem in den D-Zellen des Magen-Darm-Trakts, aber auch in Thymus, Nebenniere, Niere, Gehirn, Hypophyse, Schilddrüse, Milz, Zellen des peripheren Nervensystems sowie Zellen der Entzündungs- und Immunreaktion produziert (Csaba und Dournaud 2001). Es kommt in zwei unterschiedlichen molekularen Formen vor: Somatostatin-14, bestehend aus 14 Aminosäure, und Somatostatin-28, welches sich aus 28 Aminosäuren zusammensetzt. Beide Moleküle stellen zyklische cysteinhaltige Tetradecapeptide dar.

Sekretorische endokrine Zellen präsentieren fünf spezifische Plasmamembran-Rezeptoren, an die Somatostatin mit hoher Affinität bindet. Sie werden Somatostatin-Rezeptor (sst) 1-5 genannt (Moller et al. 2003). Fast 80 \% der gastrointestinalen NETs exprimieren SomatostatinRezeptoren (Strosberg et al. 2011 a). Sie bestehen aus einer Polypeptidkette mit sieben Transmembrandomänen und gehören zu der Familie der G-Protein-assoziierten Rezeptoren. Für die Sekretionshemmung spielen die Rezeptoren sst $_{2}$ und sst $_{5}$ die wichtigste Rolle. Der antiproliferative Effekt von Somatostatin ist vor allem auf $s^{2} t_{2}$ zurückzuführen. Dies ist zu- 
gleich der von NETs des Magen-Darm-Trakts am häufigsten exprimierte Rezeptor (Öberg 2010).

Somatostatin des Hypothalamus reguliert außerdem die Freisetzung von Wachstumsfaktoren aus der Hypophyse. Durch die dadurch entstandene Sekretionshemmung verschiedener für das Tumorwachstum wichtige Stoffe, hat Somatostatin über die Aktivierung aller Rezeptorsubtypen also auch einen indirekten antiproliferativen Effekt. Zu diesen für das Tumorwachstum wichtigen Stoffe zählen unter anderem IGF-1, verschiedene topische Hormone sowie Botenstoffe von Angiogenese und Immunsystemmodulation (Grozinsky-Glasberg et al. 2008).

Da Somatostatin jedoch eine sehr geringe Halbwertzeit von weniger als drei Minuten besitzt, wurden für den therapeutischen Einsatz chemische Analoga mit längerer Halbwertzeit entwickelt. Diese Somatostatin-Analoga (SAA) stellen in der Therapie von NETs aktuell die Therapie der ersten Wahl dar (Pavel et al. 2012). Sie sind vor allem effektiv in der Symptomkontrolle von Karzinoid-, Verner-Morrison- und Glukagonom-Syndrom, da die Expression der Somatostatin-Rezeptoren hier besonders hoch ist (Arnold et al. 2002).

Bauer und Schmidt (1980) synthetisierten Octreotid als erstes SAA. Durch das Hinzufügen dreier unnatürlicher Aminosäuren konnte die Halbwertzeit dieses Analogons auf 3-4 Stunden verlängert werden. Octreotid-LAR, ein langwirksames Analogon, besitzt eine noch längere Halbwertzeit. Dadurch konnte es möglich gemacht werden, die Injektionen von subkutan dreimal täglich auf intramuskuläre monatlich umzustellen. Octreotid wirkt vor allem an sst ${ }_{2}$ und sst $_{5}$ und hat eine moderate Affinität zu sst 3 . Unter der Therapie mit Octreotid wurden eine deutliche Abnahme der Symptomatik und eine Abnahme der 5-HIES-Ausscheidung um bis zu $50 \%$ beobachtet (Rubin et al. 1999). Der Mechanismus der Symptomlinderung ist bis heute nicht vollständig verstanden. Sicher beteiligt sind jedoch folgende Effekte: Inhibition von Hormonsekretion, Erhöhung der Wasser- und Elektrolytresorption (Dueno et al. 1987; Rubin et al. 1999) und Verminderung des Blutflusses im Splanchnikusgebiet. Durch den Wegfall dieser pathophysiologischen Mechanismen wird auch das Auftreten einer Karzinoid-Krise unwahrscheinlicher. Der Einsatz von Octreotid kann die Anzahl bedrohlicher KarzinoidKrisen senken (Dierdorf 2003). Die PROMID-Studie (2009) hat außerdem einen antiproliferativen Effekt von Octreotid-LAR bei Patienten mit metastasierten NETs des Midgut nachweisen können. Octreotid ist generell gut verträglich. Die häufigsten Nebenwirkungen bestehen in Schmerzen an der Injektionsstelle, Übelkeit, Flatulenz, Diarrhö, Steatorrhö und abdominalen Schmerzen. Diese Nebenwirkungen treten häufig nur in milder Form auf und können mit Andauern der Therapie verschwinden. Die klinisch wichtigste Nebenwirkung jedoch besteht 
in der vermehrten Ausbildung von Gallensteinen. So entwickeln bis zu $60 \%$ der Patienten, die eine Therapie mit SAA erhalten, Gallensteine (Arnold et al. 2000). Über den zugrundeliegenden Pathomechanismus wird vermutetet, dass SAA die postprandiale Freisetzung von Cholecystokinin (CCK) hemmen (Stolk et al. 1993). Dies würde somit zu einer verminderten prandialen Kontraktilität der Gallenblase führen. Die damit verbundene verminderte Konzentration an Gallensäuren im Duodenum lässt wiederum die häufige Nebenwirkung der Steatorrhö erklären. Besondere Vorsicht ist zudem bei Schwangeren geboten, da Octreotid die BlutPlazenta-Schranke überschreiten kann.

Lanreotid stellt durch das Einfügen von zwei unnatürlichen Aminosäuren, namentlich D- $\beta$ nal und Treoninamid, ein Retardpräparat dar. Es wird alle sieben bis 14 Tage verabreicht. Bereits 1999 konnte aufgrund der Beobachtungen einer Studie von Faiss et al. (1999) auch für Lanreotid ein antiproliferativer Effekt vermutet werden. Ein 2014 erschienener Case Report von Van Fraeyenhove et al. (2014) bestätigt diese Vermutung: Bei einer 55-jährigen Patientin, die an einem G3-NEC erkrankt war, konnte unter einer Hochdosis-Therapie mit Lanreotid eine Tumorkontrolle über 2 Jahre und 4 Monate erzielt werden.

Mit Pasireotid wurde ein neuer Multirezeptorligant entwickelt. Er besitzt eine hohe Affinität $\mathrm{zu} \mathrm{sst} \mathrm{t}_{1-3}$ (Schmid und Schoeffter 2004) und hat ein verbessertes Bindungsprofil zu sst ${ }_{5}$. Dadurch soll eine höhere Effektivität bezüglich des Wirkungsprofils erreicht werden. Daten klinischer Phase-III-Studien hierfür liegen jedoch bezüglich der NETs bisher nicht vor.

Den Beweis für die gute Wirksamkeit von SAA erbrachte die schon erwähnte von Rinke et al. (2009) durchgeführte PROMID-Studie: In einer Placebo-kontrollierten, randomisierten und doppelblinden prospektiven Studie wurde eine antiproliferative Wirkung von Octreotid LAR gegenüber Placebo nachgewiesen: Nach 6 Monaten war ein deutlicher Vorteil in der Octreotid LAR-Gruppe gegenüber der Placebo-Gruppe zu sehen: Nur 10 von 42 Patienten (24\%) unter Octreotid LAR gegenüber 23 von 42 Patienten (59\%) unter Placebo wiesen eine Tumorprogression auf. stable disease zeigte sich bei 28 von 42 (67\%) unter Octreotid LAR gegenüber 16 von 43 (38 \%) unter Placebo. Auch die Symptomatik nahm unter Octreotid LAR deutlicher ab als unter Placebo. Eine Normalisierung des Tumormarkers CgA konnte unter Octreotid LAR häufiger beobachtet werden als unter Placebo. Lediglich hinsichtlich der Regression konnte kein Vorteil von Octreotid LAR festgestellt werden. Diese Effekte konnten vor allem bei Patienten mit metastasierten NETs des Midgut, die eine geringe Tumorlast und einen resezierbaren Primärtumor bei Erstdiagnose aufwiesen, gezeigt werden. (Zur detaillierteren Beschreibung der Studie vgl. auch Kapitel 4.3.) 
Einen weiteren Beweis für die antiproliferative Wirksamkeit von SAA erbrachte die CLARINET-Studie, in der der Erfolg einer Therapie mit Lanreotid-Autogel untersucht wurde (Caplin et al. 2014). Auch hier handelt es sich um eine randomisierte, doppelblinde, Placebokontrollierte und multinationale Studie. Im Unterschied zur PROMID-Studie von Rinke et al. (2009) wurden hier Patienten mit fortgeschrittenen, gut- bis mäßig-differenzierten, nichtfunktionellen NETs, deren Ursprung im Pankreas, im Mid- oder Hindgut lag oder nicht bekannt war, beobachtet. Bezüglich des progressionsfreien Überlebens zeigte sich eine deutliche Überlegenheit von Lanreotid gegenüber Placebo: Nach 24 Monaten waren 65,1 \% der Patienten unter Lanreotid progressionsfrei, wohingegen nur 33,0 \% der Placebogruppe noch keine Progression aufwiesen.

Ein bisher ungelöstes Problem in der Langzeittherapie mit SAA stellt die Tachyphylaxie dar. Unter Tachyphylaxie versteht man das Nachlassen der Wirkung eines Medikaments nach einer bestimmten Zeit. Bei Octreotid und Lanreotid ist dies nach ungefähr 6-18 Monaten Behandlungsdauer der Fall. Es wird vermutet, dass die Phosphorylierung (Hipkin et al. 1997) und Internalisierung (Beaumont et al. 1998) von Rezeptoren hierbei eine große Rolle spielen. Ein weiteres beobachtetes Phänomen ist die Bildung von Antikörpern gegen SomatostatinRezeptoren, die, wie von Kaal et al. (2000) beobachtet, auch entscheidend von der Applikationsart abhängt: Bei nasaler Gabe von Octreotid war ein Nachweis von Antikörpern nach 9-12 Monaten in bis zu $81 \%$ der Patienten positiv. Bei subkutaner Gabe von Octreotid waren weniger Patienten Antikörper-positiv, wobei die Zahl der Antikörper-positiven Patienten in derselben Studie mit zunehmender Therapiedauer anstieg. Bei intramuskulärer Gabe konnten auch nach einer Behandlungsdauer von 2,5 Jahren keine Antikörper nachgewiesen werden.

Unabhängig vom zugrundeliegenden Pathomechanismus führt eine Tachyphylaxie zu einer verminderten Inhibition von Hormonfreisetzung und damit zum Ausbleiben der Symptomkontrolle. Durch das damit erhöhte Risiko zur Ausbildung einer Karzinoid-Krise oder eines Karzinoid-Herzschadens sind somit auch Mortalität und Morbidität durch das Auftreten einer Tachyphylaxie erhöht. Patienten mit Leberzirrhose oder portaler Hypertension scheinen zu einer schnelleren Resistenzentwicklung zu neigen, was vor allem daran liegt, dass der vasoaktive Effekt auf Portal- und Azygosgefäße nach anfänglicher Minderung im Vergleich zu Patienten ohne Leberbeteiligung schneller wieder zunimmt (Escorsell et al. 2001). Pasireotid soll durch sein verändertes Wirkungsprofil auch zu einem geringeren Wirkungsverlust führen. Öberg (2010) schrieb ihm deshalb in seinem 2010 veröffentlichten Symposium eine erfolgsversprechende Rolle in der Behandlung bereits Octreotid-resistenter Tumoren zu. 


\subsubsection{Biotherapie mit Interferon- $\alpha$}

Die Biotherapie umfasst neben der Anwendung von SAA auch den Einsatz von Interferon- $\alpha$, das, wie im Folgenden ausgeführt, sowohl zur Reduktion der Hormonsekretion, als auch zur Induktion einer antiproliferativen Wirkung führt.

Die Kombitherapie aus Octreotid und Interferon- $\alpha$ (IFN $\alpha$ ) bringt Vor-, jedoch auch Nachteile mit sich, die im Folgenden aufgezeigt werden sollen: Durch Interferon- $\alpha$ wird die Expression von Somatostatin-Rezeptoren verstärkt induziert (Hofland et al. 1999), was die Bedingungen einer Therapie mit SAA begünstigt. Interferon- $\alpha$ wirkt durch Stimulation der T-Zellen immunmodulativ und hat einen inhibitorischen Effekt auf die Angiogenese (Scoazec 2013). AuBerdem wirkt es antiproliferativ, indem es einen Zell-Zyklus-Arrest in der $\mathrm{G}_{1}$ - oder $\mathrm{G}_{0}$-Phase induziert und zu einer vermehrten Expression von bcl-2, einem Regulatorprotein der Apoptose, führt. Dieser Effekt wurde von Öberg et al. (2004) beschrieben. Sie publizierten einen höheren antiproliferativen Effekt unter der Therapie mit Interferon- $\alpha$, wo eine Tumorregression in 10-15\% der Fälle beobachtet werden konnte, verglichen mit der alleinigen Therapie mit SAA, worunter nur selten eine Regression der Tumormasse auftritt. Auch konnte von Kölby et al. (2003) eine Verbesserung der 5-Jahres-Überlebensrate bei Patienten mit in die Leber metastasierten funktionellen NETs des Midgut nachgewiesen werden: Verglichen mit einer SAA-Monotherapie stieg die Rate der Überlebenden nach 5 Jahren von 37 \% unter Monotherapie auf $57 \%$ unter Kombitherapie, wobei der Vergleich jedoch keine statistische Signifikanz aufwies. Bezüglich der Symptomkontrolle konnten Creutzfeldt et al. (1991) zeigen, dass eine Therapie mit SAA der Therapie mit Interferon- $\alpha$ überlegen ist.

Der Nachteil dieser Therapie besteht in einem deutlich späteren Wirkungseintritt und einem ungünstigeren Nebenwirkungsprofil: Unter Interferontherapie wurden grippeähnliche Symptome, Müdigkeit, Appetitlosigkeit, Gewichtsverlust, depressive Verstimmungen und das Auftreten von Panzytopenie beobachtet. Die Therapie mit Interferonen stellt daher trotz der erfolgsversprechenden antiproliferativen Wirkung die Zweitlinientherapie bei langsam wachsenden NETs dar (Eriksson et al. 2008). Als Kontraindikation gelten ein Alter über 70 Jahre und eine vorhandene autoimmune oder psychische Erkrankung. Vorsicht ist außerdem während der Stillzeit geboten, da Interferon- $\alpha$ in Muttermilch nachgewiesen werden konnte (Öberg et al. 2009).

\subsubsection{Therapie mit neuen Pharmaka}

Everolimus, ein m-TOR-Inhibitor (m-TOR = mamalian taget of Rapamycin), der einmal täglich oral verabreicht wird, bindet FKBP-12 und ist somit für die Regulation der Proteinsynthese in gesunden wie malignen Zellen verantwortlich, da vor allem Angiogenese, Zellwachs- 
tum und -proliferation und Zellmetabolismus des Tumors über FKBP-12 gesteuert werden. Neue Studien weisen auf einen antiproliferativen Effekt von Everolimus hin: Eine von Yao et al. (2011) veröffentlichte Studie konnte eine Verlängerung der progressionsfreien Überlebenszeit in fortgeschrittenen G1- und G2- PNETs unter Therapie mit Everolimus nachweisen. Hier betrug die mediane progressionsfreie Überlebenszeit der 207 Patienten unter Everolimus 11 Monate verglichen mit einer medianen progressionsfreien Überlebenszeit von 4.6 Monaten bei 203 Patienten unter Placebo, was einer Reduktion des Progressionsrisikos von $65 \%$ entspricht.

Sunitinib, ein Multitarget-Tyrosin-Kinase-Inhibitor, wirkt antiproliferativ und inhibiert die Vaskularisierung des Tumors. Es führt bei Patienten mit fortgeschrittenem, gutdifferenziertem PNETs zu einer Verlängerung der progressionsfreien Überlebenszeit. Dies wurde von Raymond et al. (2011) zwischen 2007 und 2009 in einer Placebo-kontrollierten Phase-III-Studie untersucht: Verglichen mit Placebo, worunter die mediane progressionsfreie Überlebenszeit der 85 Patienten 5,5 Monate betrug, zeigte sich bei den 86 Patienten unter Sunitinib eine mediane progressionsfreie Überlebenszeit von 11,4 Monaten. Im Langzeitverlauf zeigte sich eine Wahrscheinlichkeit für das Überleben von 6 Monaten, die in der PlaceboGruppe bei 85,2 \%, in der Sunitinib-Gruppe jedoch bei 92,6 \% lag. Limitiert wird diese Therapie allerdings durch eine Abnahme der Lebensqualität, welche durch das Nebenwirkungsprofil von Sunitinib, wozu Diarrhö, Müdigkeit, kardiotoxische Wirkungen, Blutdruckanstieg und Thrombozytopenie gehören, bedingt ist.

Andere Angiogeneseinhibitoren wie Sorafinib (Tyrosin-Kinase-Inhibitor) und Bevacizumab (Inhibitor des Vasculary-endothelial-groth-factor (VEGF)) haben als Monotherapie bisher nur mäßige Erfolge erbracht (Öberg 2010).

\subsubsection{Chemotherapie}

Eine weitere medikamentöse Therapieoption stellt die Chemotherapie dar. Da die meisten NETs ein langsames Wachstum vorwiesen, sind sie der Chemotherapie nur schwer zugänglich. Der Erfolg einer Chemotherapie ist von der Lokalisation des Primärtumors und dessen Grading abhängig. Bei einem G1- oder G2-Tumor mit schneller Progression oder hoher systemischer Ausbreitung wird eine Chemotherapie mit 5-Fluorouracil + Streptozotocin empfohlen. G3-Tumoren sollten mit einer Kombination aus Cisplatin + Etoposid therapiert werden (Pavel et al. 2012).

Daraus lässt sich schließen, dass der Differenzierungsgrad und die Wachstumsgeschwindigkeit des Tumors eine wichtige Rolle in der Therapieentscheidung zu einer Chemotherapie spielen. Patienten mit schnellem Tumorwachstum und hohem Grading werden eher von einer 
Chemotherapie profitieren, während SAA für Patienten mit einem langsamen Tumorwachstum und niedrigem Grading durchaus die bessere Therapieoption darstellen (Öberg 2010).

\subsubsection{Radiotherapie}

NETs sprechen nur schlecht auf Radiotherapie im Sinne einer externen Bestrahlung an. Die einzige Indikation für eine solche Bestrahlung stellt die palliative Bestrahlung von Metastasen in der Wirbelsäule dar.

Ein weiteres Feld der bei NETs angewandten Radiotherapie stellt die Therapie mit radioaktiv markierten SAA dar: Unter dieser Therapie konnten Symptomkontrolle und eine Stabilisation des Tumorwachstums in $40 \%$ der Patienten nachgewiesen werden (Öberg 2010). Hierbei werden radioaktive Stoffe mittels Chelatoren an SAA gebunden, wodurch eine Bindung der radioaktiven Stoffe an die Somatostatin-Rezeptoren möglich wird. Die so aufgenommenen radioaktiven Stoffe entfalten durch die Verstoffwechslung in der Zelle ihre Wirkung und führen so zu einer selektiven Zerstörung des Tumorgewebes. Die am meisten verwendeten radioaktiven Stoffe sind die $\beta$-Strahler ${ }^{90}$ Yttrium, ${ }^{111}$ Indium und ${ }^{177}$ Lutetium. Als Chelatoren dienen Polyaminopolycarboxid-Säuren oder deren makrozyklische Derivate wie DOTA, TETA, TTHA oder DTPA (Ausgeschriebene Namen siehe Abkürzungsverzeichnis). Gemäß dem Bindungsprofil von Octreotid wirken diese Stoffe vor allem durch die Bindung an $\mathbf{s s t}_{2}$. Der Erfolg der Radionukleidtherapie hängt also wesentlich von der Expressionsdichte und Affinität der Somatostatin-Rezeptoren ab. Die Indikation einer solchen Therapie wird vor allem durch Szintigraphie nach Gabe eines radioaktiv markierten Octreotids (OctreoScan) gestellt. Radioaktiv markiertes Octreotid wird vor allem in der Leber verstoffwechselt, was im OctreoScan zu einem physiologischen Signal der Leber führt. Für die Entscheidung zu einer Radionukleidtherapie ist die Stärke des vom Tumor gesendeten Signals maßgebend. Es sollte mindestens so hoch wie das normale Lebersignal sein.

Kontraindikationen stellen Schwangerschaft und Stillzeit, Nieren-, Knochenmarks-, Leberoder Herzinsuffizienz dar. Um einen größeren Effekt zu erzielen sollte eine Therapie mit langwirksamen SAA rechtzeitig vor der Radionukleidtherapie pausiert werden, da diese aufgrund der kompetitive Bindung mit dem Radionukleid um den Bindungsplatz am Rezeptor konkurrieren würden, was dessen Wirkung abschwächen könnte. ${ }^{\left[{ }^{177} \mathrm{Lu}-\right.}$ DOTA $^{0}$, Tyr]Octreotid wird am häufigsten eingesetzt. Es liegen jedoch keine Studien zum Vergleich verschiedener radioaktiv markierten SAA vor. Ebenso unsicher ist die Datenlage bezüglich der Langzeitfolgen (Kwekkeboom et al. 2009). 


\subsubsection{Unterstützende symptomatische Therapie}

Palliativ können eine Reihe von unterstützenden Therapien zum Einsatz kommen. Neben Schmerztherapie und palliativer Radiotherapie bei Knochenmetastasen der Wirbelsäule stellen auch spezifische Therapien zur Kontrolle der hormonell verursachten Symptome eine Therapieoption dar. So kann beispielsweise ein Zollinger-Ellison-Syndrom durch die Gabe von Protonenpumpeninhibitoren unterstützend therapiert werden. Bei Insulinomen kommt Diazoxid zur Therapie der Hypoglykämie zum Einsatz (Arnold et al. 2000). Schwörer et al. (1995) zeigten in einem Case Report die erfolgreiche Therapie von durch Karzinod-Syndrom hervorgerufener Diarrhö mit 5-HT 3 -Rezeptorantagonisten sowie Alpha $2^{-}$ Rezeptorantagonsiten.

\subsection{Prognose}

In einer von Yao et al. (2008) durchgeführten Studie, in der NETs aller Differenzierungsgrade, Lokalisationen und Ursprünge eingeschlossen worden waren, konnte eine generelle mittlere Überlebensdauer von 75 Monaten erreicht werden. In derselben Studie konnte jedoch auch gezeigt werden, dass die Dauer des Überlebens wesentlich von der Tumorlokalisation und dem Tumorgrading abhängt: Während die mittlere Überlebenszeit bei Patienten mit G3Tumoren 10 Monate betrug, konnte bei Patienten mit G1-Tumoren eine mittlere Überlebenszeit von 129 Monaten erreicht werden. NETs des Dünndarms wiesen außerdem eine wesentlich bessere Prognose auf als NETs, deren Ursprung im Kolon zu finden war. Als prognostische Faktoren gelten neben Lokalisation des Primärtumors, Grading und Staging auch das Vorhandensein tumorspezifischer Symptome, Expression von Somatostatin-Rezeptoren und deren Dichte, der Verlauf verschiedener Tumormarker sowie die Expression verschiedener Protoonkogene wie p53. Auch das Alter, Geschlecht und die Herkunft des Patienten sowie die Dauer seit der Erstdiagnose scheinen einen prognostischen Faktor darzustellen (Yao et al. 2008). Weiterhin wichtig für die Prognose ist die Tumorgröße. Verschiedene Studien haben gezeigt, dass diese mit der Frequenz der Metastasierung korreliert (Moertel et al. 1961; Burke et al. 1997). Bei bereits vorhandener Metastasierung spielen außerdem Art, Anzahl und Lokalisation der Metastasen eine prognostische Rolle (Pavel et al. 2012). Als stärkste negative prognostische Faktoren haben sich Alter des Patienten, ein fortgeschrittenes Staging und ein niedriger Differenzierungsgrad herausgestellt (Halfdanarson et al. 2008). 


\subsection{Zielsetzung der Arbeit}

Die Therapie von NETs ist vielseitig. SAA haben historisch gesehen vor allem einen Stellenwert in der Symptomkontrolle, wo sie seit langem mit gutem Erfolg eingesetzt werden. Erste Studien, wie beispielsweise die von Arnold et al. (1996) oder von Rinke et al. (2009), zeigen neben dem Effekt der Symptomkontrolle auch einen Effekt auf das Tumorwachstum, in dem sie eine längere Dauer der stable disease unter der Therapie mit SAA nachweisen konnten (detaillierte Vorstellung der genannten Studien siehe Kapitel 4.3, zur Definition der stable disease siehe Kapitel 2.2.3). Bisherige Studien untersuchten außerdem allenfalls einen mittelfristigen Verlauf mit Beobachtungsdauern von bis zu 27 Monaten (CLARINET-Studie, Caplin et al. 2014). Bisher fehlen daher Daten zum Langzeitverlauf eines SAA-basierten Therapieregimes.

Ziel der von uns durchgeführten retrospektiven Analyse ist es, den möglichen antiproliferativen Effekt von SAA anhand der Effekte auf Tumorgröße und Metastasierung auch in unserem Patientenkollektiv, das über einen längeren Zeitraum mit SAA behandelt wurde, hier durchschnittlich 47,68 Monate, nachzuweisen. Auch die Frage nach der Abhängigkeit dieser Effekte vom initialen Metastasierungsstadium und die Effekte einer solchen Therapie auf die Ausbildung und den Verlauf von Metastasen, unabhängig vom Verlauf des Primärtumors, sollen hier untersucht werden. Des Weiteren gilt es, den positiven Effekt von SAA auf die Kontrolle der Symptomatik und die Kontrolle der Tumormarker zu belegen und somit die wichtigsten Punkte eines Therapieerfolgs (siehe Kapitel 1.3) nachzuweisen. Da der Beobachtungszeitraum unserer Analyse nicht nur den Zeitraum einer SAA-Monotherapie beinhaltet, sind mögliche Effekte einer Zusatztherapie ebenso Bestandteil dieser Arbeit. Ziel hierbei ist es, herauszufinden, ob ein zusätzlicher Einsatz von Chemotherapie, Interferon- $\alpha$ oder nuklearmedizinischen Methoden einen zusätzlichen Effekt auf den Verlauf der Tumorerkrankung hat. 


\section{Methodik}

Zwischen 1. Januar 1975 und 31. August 2011 wurden insgesamt 66 Patienten mit histologisch gesicherten NET unterschiedlicher Lokalisation, die in der Universitätsmedizin Göttingen betreut und mit SAA behandelt wurden, beobachtet und in die hier vorliegende Analyse eingeschlossen.

Die Behandlung mit SAA verlief dabei wie folgt: Die Entscheidung zu einer Therapie mit SAA erfolgte anhand der gezeigten Symptomatik. In der Regel wurde mit einer Dosis von 50 $\mu$ g s.c. $3 \mathrm{x}$ täglich begonnen. Anhand der Symptomkontrolle wurde die jeweilige Dosis von SAA auftitriert. Bei drei der Patienten lag die Höchstdosis an SAA zum Zeitpunkt der Progression bei $500 \mu \mathrm{g}$ s.c. 3 x täglich, was dem Zehnfachen der Anfangsdosis entspricht. Bei einigen der Patienten wurde im Laufe der Zeit auf ein Monatspräparat, das i.m. verabreicht werden konnte, umgestellt. Die höchste auf diese Art verabreichte Dosis an SAA beträgt in unserem Patientenkollektiv $30 \mathrm{mg}$ monatlich (bei sechs Patienten), was einer Tagesdosis von $1000 \mu \mathrm{g}$, also dem fast Siebenfachen der Anfangsdosis entspricht. Hierbei handelt es sich um das langwirksame Präparat Octreotid-LAR. Insgesamt hatten die von uns analysierten Patienten bei Progression eine Höchstdosis zwischen $50 \mu$ g s.c. 3 x täglich, also einer Dosis von 150 $\mu \mathrm{g} / \mathrm{d}$ (vor allem bei Patienten, die eine sehr kurze Dauer des stable disease vorwiesen) und $1500 \mu \mathrm{g} / \mathrm{d}$ erhalten. Die mittlere verabreichte Höchstdosis betrug in unserem Kollektiv 527 $\mu \mathrm{g} / \mathrm{d}$.

\subsection{Klassifikation}

Im Rahmen der primären Diagnostik wurden ein Grading sowie ein Staging nach der TNMKlassifikation durchgeführt.

\subsubsection{Grading}

Der Parameter, der zum Grading herangezogen wird, ist der Ki-67-Index. Da er von der WHO als wichtigstes Kriterium für Tumorgrading, Prognose und Therapieentscheidung gesehen wird (Remes et al. 2012) richtet sich hiernach auch die momentane WHO-Klassifikation (G1: $\leq 2 \%$; G2: 3-20\%; G3: < $20 \%$ ). Dieser liegt auch dem in dieser Analyse verwendetem Grading zugrunde. Zusammen mit der Anzahl der Mitosen pro Quadratmillimeter, die histopathologisch unter dem Mikroskop ausgezählt werden können, ergeben sie ein wie in Kapitel 1.1.3 beschriebenes Grading nach der aktuellen WHO-Klassifikation. Das Grading ist also ein Wert, der sich nur durch eine mikroskopische Betrachtung von Gewebeproben bestimmen lässt. Unabdingbar für die Bestimmung des Gradings ist damit ein operativ gewonnenes Tu- 
morresektat oder eine im Zuge einer endoskopischen Untersuchung bioptisch gewonnene Gewebeprobe.

Bestimmung des Ki-67-Index:

Ki-67 ist ein Antigen, welches in der DNA aller sich teilenden Zellen zu finden ist (Gerdes et al. 1991). Cattoretti et al. (1992) beschrieben eine Methode, mit der sich das Ki-67-Antigen in sich teilenden Zellen nachweisen lässt: Entwickelt wurden drei monoklonale Antikörper, MIB1-3, gegen rekombinante Anteile des Ki-67. Durch die Bindung von MIB an Ki-67 können entsprechende DNA-Abschnitte in mitotisch aktiven Zellen immunhistochemisch sichtbar gemacht werden. Dabei wird MIB auf in Formalin fixierten und in Paraffin gebetteten Zellen bestimmt. Immunhistochemisch ergibt sich nun in mitotisch aktiven Zellen ein Signal, wohingegen Zellen, die sich in der $\mathrm{G}_{0}$-Phase befinden, kein Signal senden. Daraus lässt sich schließen, dass neoplastische, teilungsaktive Zellen auf MIB1 positiv reagieren, wohingegen das Signal bei gesunden Zellen negativ ausfällt (Catoretti et al. 1992). Vor allem MIB1 wird in heutigen histopathologischen Untersuchungen eingesetzt. Mikroskopisch wird an die Färbung anschließend der Bereich, in dem die höchste Signalaktivität nachweisbar ist, angeschaut. Der Anteil der Ki-67 positiven Zellen unter 2000 Tumorzellen in diesem Areal bestimmt die für das Grading verwendete Prozent-Zahl (Panzuto et al. 2011).

\subsubsection{Staging}

Nach der TNM-Klassifikation der UICC wird das Grading durch das T-Stadium (= die Infiltrationstiefe), das N-Stadium (= die Anzahl befallener Lymphknoten) sowie das M-Stadium (= die systemische Ausbreitung) bestimmt (Sobin et al. 2009).

Die Infiltrationstiefe, also das T-Stadium, kann im Resektionspräparat histologisch bestimmt werden. Eine präoperative Einschätzung ist an endoskopisch zugänglichen Stellen mittels Endosonographie möglich (Riemann et al. 2010). Ebenso kann diese intraoperativ, makroskopisch geschätzt werden. Der Befall regionaler Lymphknoten (N-Stadium) ist intraoperativ im Zuge einer Tumorresektion unter Mitnahme regionaler Lymphknoten, die anschließend histopathologisch ausgewertet werden, nachweisbar. Vereinzelt kann der Verdacht auf einen Befall der Lymphknoten auch schon in der Endosonographie gestellt werden (Riemann et al. 2010). Ist der Lymphkontenbefall bereits ausgeprägt, kann er auch in CT und MRT darzustellen sein. Die systemische Ausbreitung des NETs, das M-Stadium, wird mittels Computertomographie (CT), Magnetresonanztomographie (MRT) und Sonographie des Abdomens bestimmt (Öberg und Castello 2011), wobei nach metastaseverdächtigen Läsionen gesucht wird.

Die Einteilung in die verschiedenen Tumorstadien erfolgt gemäß der aktuellen TNMKlassifikation wie im Kapitel 1.1.4 beschrieben. 


\subsection{Diagnostik}

Die Diagnostik erfolgte mittels Sonographie, Endoskopie und Endosonographie, Computertomographie, Magnetresonanztomographie sowie Somatostatin-Rezeptor-Szintigraphie (SRS).

\subsection{Verlaufskontrolle}

Alle 66 in diese Analyse eingeschlossenen Patienten wurden im Sinne einer Verlaufskontrolle regelmäßig in folgenden Abständen untersucht: Patienten unter Chemotherapie nach jedem dritten Zyklus (in der Regel entsprechend einer Kontrolle alle drei Monate), Patienten unter Therapie mit SAA und Progredienz alle drei Monate, Patienten unter SAA, die eine stable disease aufwiesen bzw. Patienten, die zum gegebenen Zeitpunkt keine Therapie bekamen, alle sechs Monate. Jede dieser Untersuchungen zur erneuten Beurteilung des Verlaufs beinhaltete eine Kontrolle der Tumormarker, die Beurteilung des Primärtumors mittels endoskopischen Verfahren wie Gastroskopie und Koloskopie und ein erneutes Staging mittels bildgebender Verfahren wie CT, MRT und Sonographie des Abdomens.

Mussten Patienten regelmäßig zur Verabreichung ihrer Medikation erscheinen, was vor allem bei subkutan und intramuskulär verabreichten Präparaten der Somatostatin-Analoga oder im Rahmen einer Chemotherapie der Fall war, wurde im Zuge dieses Termins eine Anamnese und klinische Untersuchung durchgeführt. Zusätzliche ausführliche Anamnese und Untersuchung fanden zu den jeweiligen Zeitpunkten der Verlaufskontrolle (siehe oben) statt. Innerhalb der Anamneseerhebung wurden die Patienten nach ihrer derzeitigen Symptomatik, vor allem bezüglich eines Karzinoid-Syndroms (Flush, Diarrhö) und einer drohenden kardialen Mitbeteiligung (Zeichen der Herzinsuffizienz) befragt. Eine weitere wichtige Rolle in diesen Anamnesegesprächen spielt das subjektive Wohlbefinden und die Verträglichkeit der Therapie (Nebenwirkungen wie Abgeschlagenheit, Schmerzen, neurologische Symptome (vor allem bei angewandter Chemotherapie)). Daten, die in unserer Analyse den Verlauf der Symptomatik nachzeichnen sollen, basieren auf diesen Patientenbefragungen. Zur Quantifizierung wurde bei vorhandener Diarrhö zusätzlich das Stuhlgewicht bestimmt.

\subsubsection{Tumormarker}

Zur Verlaufskontrolle wurden Chromogranin A (CgA), 5-Hydroxyindolessigsäure (5-HIES), Neuronspezifische Enolase (NSE) und Serotonin verwendet. Wie bereits in Kapitel 1.2.3 er- 
wähnt, kann CgA unabhängig von der endokrinen Aktivität der Tumorzellen im Blut des Patienten oder per Immunhistochemie direkt im Gewebe nachgewiesen werden (Rindi et al. 2004) und ist somit ein direkter Parameter für die vorhandene Tumorlast (Pirker et al. 1998). 5HIES, NSE und Serotonin hingegen sind jeweils nur in funktionellen NETs nachweisbar, da sie mit der Menge der von den Tumorzellen ausgeschütteten Hormone bzw. deren Abbauprodukte korrelieren. Die am längsten bekannten und für die Verlaufskontrolle von NETs verwandten Marker sind 5-HIES und Serotonin. Erst um 1995 wurden die Marker NSE und CgA entdeckt und deren Nutzen erkannt. Gleichzeitig nahm damit die diagnostische Bedeutung von 5-HIES und Serotonin ab. Dies hat für die vorliegende Analyse zur Folge, dass zu den wenigsten Zeiten der Verlauf aller vier Tumormarker nachvollzogen werden kann.

Kaltsas et al. (2002) veröffentlichten in einer Beobachtung zur Therapie von NETs ein standardisiertes Vorgehen zur Beurteilung des Verlaufs von Tumormarkern.

Folgende daraus entstandene Einteilung kommt auch in unserer Analyse zur Anwendung:

- Ein komplettes Ansprechen (complete response) auf die Therapie liegt dann vor, wenn es unter der Therapie zu einer Normalisierung der Werte des Tumormarkers kommt. Dies ist mit einer Remission gleichzusetzen.

- Partielles Ansprechen (partial response) entspricht einer Abnahme der Werte um mindestens $50 \%$ während der Therapie.

- Von einer Progression wird dann gesprochen, wenn die Zunahme der Werte unter der Therapie mindestens $25 \%$ des Ausgangswertes beträgt.

- Kommt es unter der Therapie weder zu einer Abnahme um mindestens $50 \%$ noch zu einer Zunahme von $25 \%$ oder mehr der Ausgangswerte, so geht man von einem nicht Ansprechen der Therapie aus (No Response).

\subsubsection{Verlauf der Tumorgröße}

Therasse et al. (2000) entwickelten neue Leitlinien, um den Verlauf solider Tumoren und deren Ansprechen auf eine Therapie beschreiben zu können. Sie wurden von Eisenhauer et al. (2009) noch einmal aktualisiert. Diese so genannten RECIST-Kriterien (RECIST = response evaluation criteria in solid tumors) liegen auch der Beurteilung des Verlaufs der Tumorgröße in dieser Analyse zugrunde:

- Ein komplettes Ansprechen (complete response) auf die Therapie liegt dann vor, wenn sämtliche zu Beginn der Therapie beschriebenen Tumorläsionen in den Verlaufsuntersuchungen verschwunden, sprich nicht mehr nachweisbar sind. Lymphkoten, die zu 
Beginn der Therapie pathologisch vergrößert erschienen, müssen auf eine Größe $<10$ mm zurückgegangen sein.

- Beträgt die längste gemessene Strecke einer Tumorläsion in der Untersuchung der Verlaufskontrolle mindestens $30 \%$ weniger als bei der initialen Untersuchung, so spricht man von einem teilweisen Ansprechen (partial response) auf die Therapie.

- Eine Progression (progressiv disease) liegt dann vor, wenn die längste gemessene Strecke der Tumorläsion in der Verlaufskontrolle gegenüber der initialen Untersuchung mindestens um $20 \%$ größer geworden ist. Von einer Progression spricht man außerdem, wenn in einer Verlaufskontrolle mindestens eine neue Läsion, zum Beispiel in Form einer neu aufgetretenen Metastase, nachgewiesen werden kann.

- Kann in der Verlaufskontrolle keine neu aufgetretene Läsion und weder eine Zunahme um $20 \%$, noch eine Abnahme um $30 \%$ der längsten gemessenen Strecke der Tumorläsion nachgewiesen werden, so geht man von einer Stabilität der Tumorerkrankung (stable disease) aus. Ihre Dauer wird vom Zeitpunkt des Therapiebeginns bis zum ersten Anzeichen einer Progression oder Abnahme gemäß der oben genannten Definition gemessen.

In unserer Analyse entspricht die Dauer der stable disease somit dem Zeitraum, in dem unter der Therapie mit SAA weder eine Progression noch eine Abnahme der Tumorgröße nach oben genannten Kriterien beschrieben werden konnte.

Der Verlauf der Tumorgröße wurde dabei mittels Bildgebenden Verfahren (CT, MRT und Sonographie) beurteilt. Die Einteilung anhand der RECIST-Kriterien erfolgte durch den befundenden Radiologen. Bei Tumorverläufen vor dem Jahr 2000 erfolgte die Einteilung in die Kategorien der RECIST-Kriterien durch uns anhand der bildmorphologisch erhobenen und in den radiologischen beziehungsweise sonographischen Befunden angegebenen Messungen der jeweiligen Läsion im Nachhinein im Rahmen der Datenerhebung für diese Analyse.

Des Weiteren wurde in dieser Analyse das Auftreten von Rezidiven beobachtet. Patienten, die hier ein Rezidiv aufwiesen, waren nach einer primär operativen Therapie radiologisch tumorfrei, wobei der Grad der chirurgischen Resektion nicht näher definiert ist. Das Auftreten eines Rezidivs beinhaltet somit das erneute Sichtbarwerden des Tumors in der Bildgebung.

In unserer Beobachtung wurde der Verlauf der Tumorgröße in zwei verschiedenen Zeiträumen verglichen: Dem Zeitraum, in dem die Patienten eine Monotherapie mit SAA erhielten und dem Zeitraum, in dem die Patienten entweder anderweitig, zum Beispiel mittels Chemotherapie, behandelt wurden oder keine Therapie bekamen. 
Zur Unterscheidung von Verlauf von Tumormarker und Verlauf der Tumorgröße kommen in der vorliegenden Analyse bei der Beschreibung der Tumormarker nach den Kriterien von Kaltsas et al. (2002) die deutschen Bezeichnungen und bei der Beschreibung der Tumorgröße nach den RECIST-Kriterien die englischen Bezeichnungen zur Anwendung.

\subsection{Wirksamkeit von SAA}

In unserer Analyse wird im Folgenden immer zwischen den Beobachtungszeiträumen mit und ohne Therapie mit SAA unterschieden. Bei den Zeiträumen mit SAA-Therapie wird des Weiteren in solche Zeiträume unterschieden, in denen eine Monotherapie mit SAA durchgeführt wurde und solche Zeiträume, in denen zusätzlich zu SAA eine alternative medikamentöse Therapie (Chemotherapie oder Therapie mit Interferon- $\alpha$ ) oder eine nuklearmedizinische Therapie durchgeführt wurde. Als Zeiträume ohne Therapie mit SAA werden sowohl die Zeiträume betrachtet, in denen gar keine Therapie durchgeführt wurde, als auch die Zeiträume, in denen eine alternative Therapie stattfand. Alternative Therapiemethoden beinhalten auch hier Chemotherapie, Interferon- $\alpha$ und nuklearmedizinische Therapie. Zur Auswertung der Verläufe sei darauf hingewiesen, dass die hierfür erhobenen Daten im gesamten Zeitintervall betrachtet werden und keinem strengen Zeit-Schema folgen.

Zur genaueren Analyse der Effekte auf Tumormarker, Tumorgröße sowie Metastasierung wurde das Patientenkollektiv zudem in zwei Gruppen eingeteilt: Eine Gruppe, die ausschließlich eine Monotherapie mit SAA erhalten hatte und eine Gruppe, bei der eine zusätzliche Therapie verwendet worden war. Bei beiden Gruppen wurde der Zeitraum mit SAA mit dem Zeitraum ohne SAA verglichen, wobei für den zuletzt genannten Zeitraum zusätzlich eine Unterscheidung in Verläufe ohne jegliche Therapie und Verläufe unter alternativer Therapie gemacht wurden. Dabei beziehen sich die genannten Zeiträume jeweils auf den gesamten Beobachtungszeitraum des jeweiligen Patienten und nicht auf ein definiert abgestecktes Intervall. Patienten, die in einem bestimmten Zeitraum ohne jegliche Therapie blieben, waren in diesem Intervall symptomfrei, so dass sich keine Notwendigkeit einer symptomorientierten Therapie ergab und/oder wiesen ein langsames Spontanwachstum auf, so dass auch eine wachstumslimitierende Therapie zu diesem Zeitpunkt nicht indiziert war.

Die Beurteilung der Effekte, die SAA auf eine Milderung der Symptomatik, eine Tumormarkerresponse oder eine Eindämmung des Tumorwachstums hatte, wurden nach den in Kapitel 2.3 genannten Kriterien im Verlauf des gesamten Zeitraums der Behandlung mit SAA (Siehe Kapitel 3.3.2) vorgenommen. Sollte es nach initialer Abnahme der Symptomatik oder Response der Tumormarker beziehungsweise der Tumorgröße zu einer erneuten Zunahme der 
Symptomatik oder einem Wiederanstieg der Tumormarker beziehungsweise erneuter Zunahme des Tumorwachstums gekommen sein, so bleibt dies unberücksichtigt.

Abbildung 1 und 2 fassen die Unterteilung der Beobachtungszeiträume beziehungsweise die Einteilung in die verschiedenen Untergruppen noch einmal visuell zusammen.

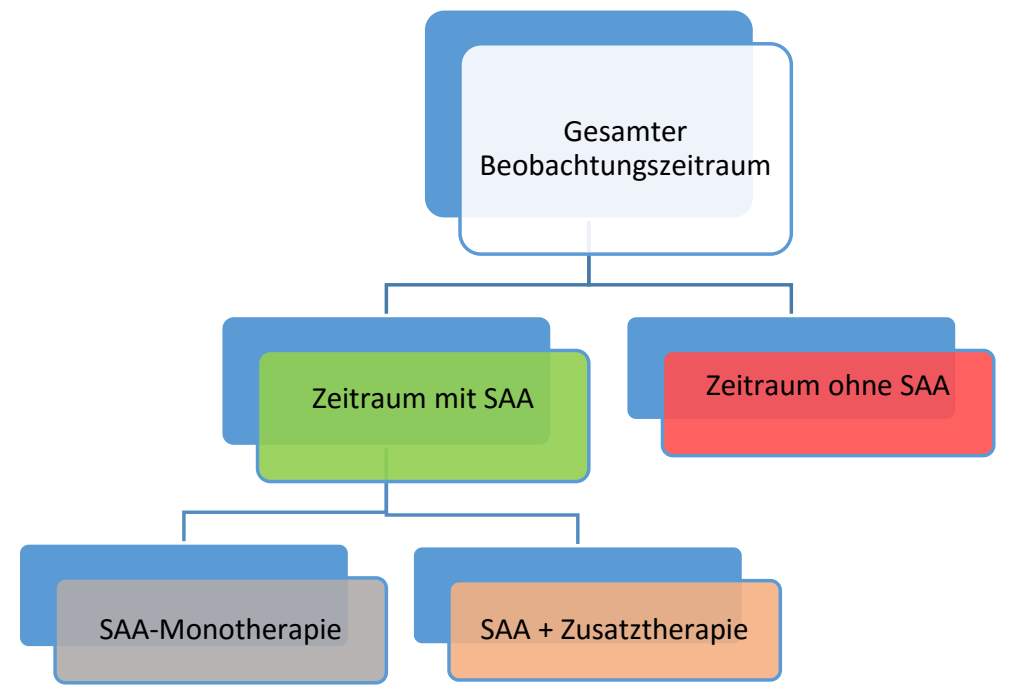

Abbildung 1: Baumdiagramm der Einteilung der Beobachtungszeiträume

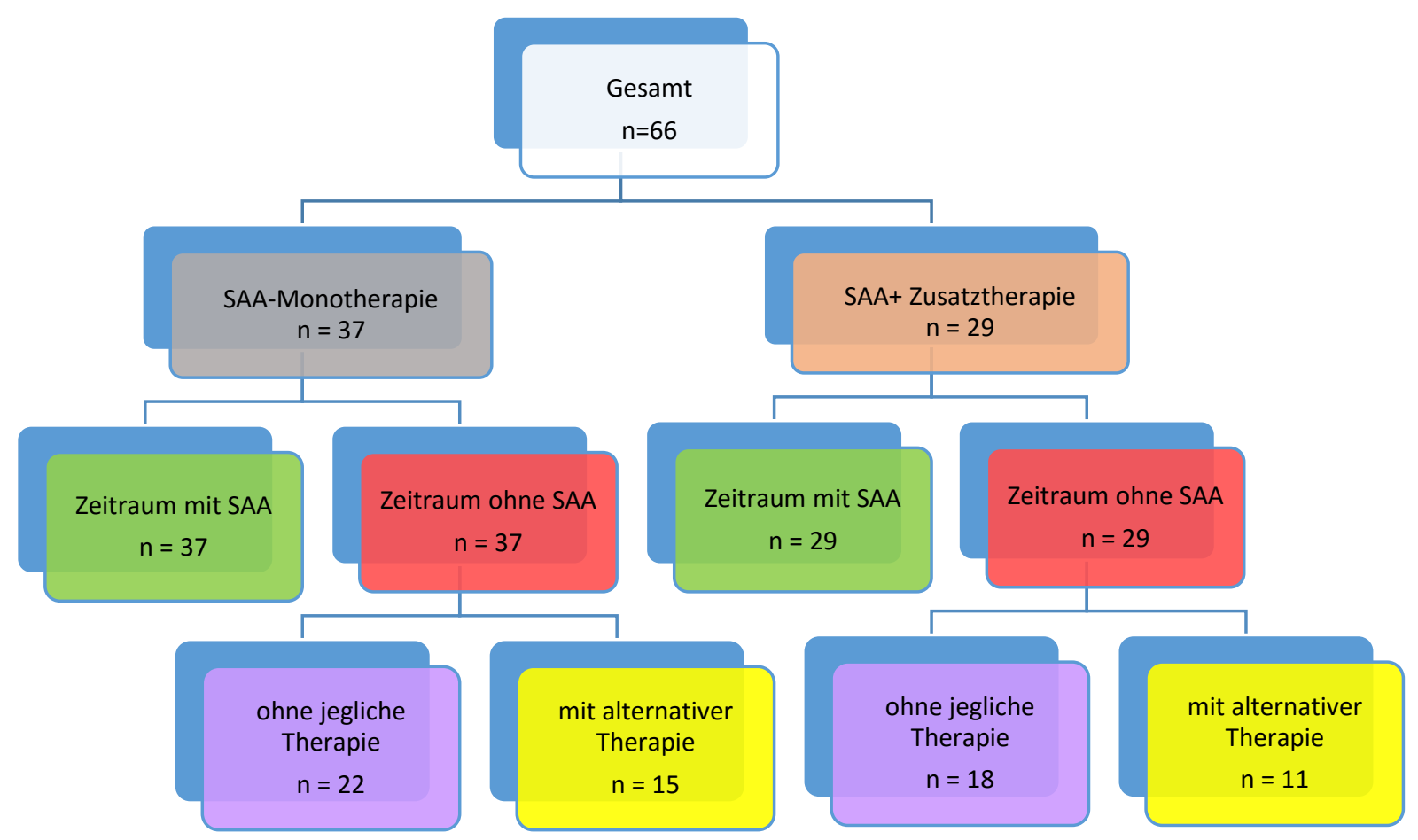

Abbildung 2: Baumdiagramm der Einteilung der zu vergleichenden Untergruppen. Im Zeitraum mit/ohne SAA werden die Patienten jeweils mit sich selbst verglichen 


\subsection{Auswertung}

Die statistische Auswertung der für diese Analyse gewonnenen Daten wurde mit Hilfe von StatSoft STATISTICA 9.1 beziehungsweise StatSoft STATISTICA 12 sowie mit Microsoft Office Excel 2007 beziehungsweise Microsoft Office Excel 2013 durchgeführt.

In der Betrachtung wurden unterschiedliche Gruppen mit einem p-Wert $<0,05$ als signifikant angesehen und als solche beschrieben. Dabei wurde für den Vergleich unterschiedlicher Gruppen untereinander jeweils der t-Test für unabhängige Gruppen angewendet.

Ein Datensatz gilt als vollständig, wenn bis einschließlich August 2011 aktuelle Befunde vorlagen. Datensätze von im Beobachtungszeitraum bis zu diesem Zeitpunkt verstorbenen Patienten wurden ebenso als vollständig betrachtet. 


\section{Ergebnisse}

\subsection{Demographie}

Im Zeitraum zwischen 1. Januar 1975 und 31. August 2011 wurden insgesamt 66 Patienten mit einem NET in der Universitätsmedizin Göttingen behandelt und beobachtet. Von diesen 66 Patienten waren 42 männlich (63,64 \%) und 24 weiblich (36,36 \%). Das Durchschnittsalter bei Erstdiagnose betrug 55,11 Jahre mit einer Standardabweichung (Stdw.) von 10,65 Jahren. Hierbei lag das Minimum bei 25 Jahren und das Maximum bei 77 Jahren. Männliche Patienten waren bei Erstdiagnose im Durchschnitt 54,57 \pm 11,17 Jahre alt, wobei sich das Minimum bei 25 Jahren und das Maximum bei 75 Jahren befand. Weibliche Patienten hatten ein durchschnittliches Alter von 56,04 \pm 9,82 Jahren mit einem Minimum von 39 Jahren und einem Maximum von 77 Jahren.

Zwischen dem Zeitpunkt der Erstdiagnose und dem Beginn einer Therapie mit SAA lagen im Mittel 3,53 \pm 12,1 Monate. Hier lag das Minimum bei 0 Monaten und das Maximum bei 50 Monaten.

Abbildung 3 zeigt die Geschlechterverteilung in dem hier vorliegenden Patientenkollektiv. Abbildung 4 veranschaulicht die Verteilung des Alters bei Erstdiagnose nach Geschlecht bei den in unsere Analyse eingeschlossenen Patienten.

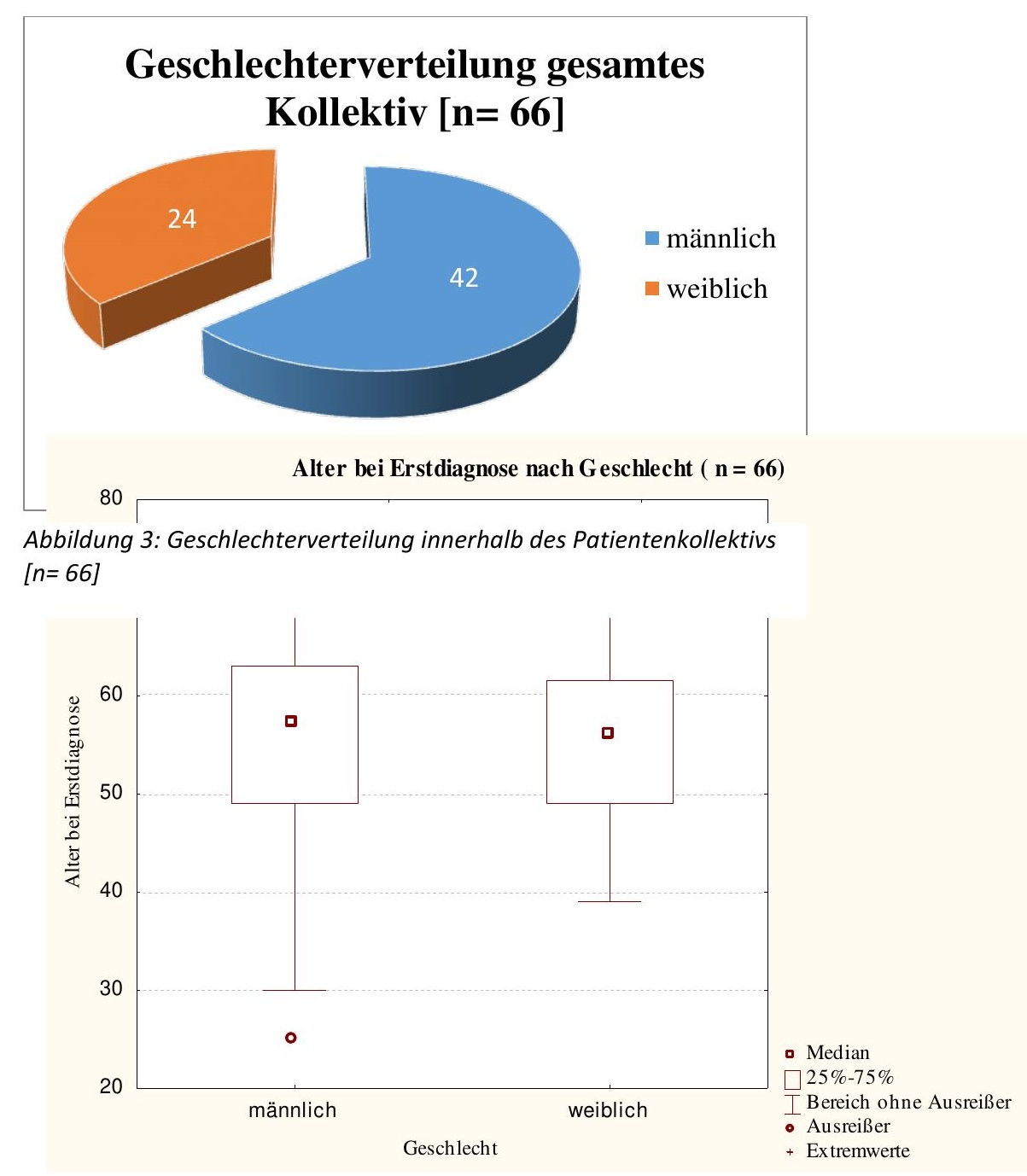


Abbildung 4: Boxplot des Alter bei Erstdiagnose nach Geschlecht geordnet. Dabei eingeschlossen sind die 24 weiblichen und 42 männlichen Patienten unseres Kollektivs [ $n=66$ ]

\subsubsection{Tumorlokalisation}

Die Lokalisation der Primärtumoren der 66 Patienten verteilte sich wie folgt: 25 im Dünndarm (37,88 \%), sechs im Kolon (9,06\%), drei in der Appendix (4,54 \%), 16 im Pankreas $(24,24 \%)$, vier in der Lunge $(6,06 \%)$, zwei im Ovar $(3,03 \%)$. Bei einem Patienten fand sich ein extraadrenales abdominelles Paragangliom (1,52\%). Bei vier Patienten fanden sich jeweils zwei Primärtumoren. Diese waren bei zwei Patienten in Dünndarm und Appendix (3,03 $\%$ ) und bei jeweils einem Patienten in Lunge und Magen (1,52 \%) bzw. Dünndarm und Pankreas (1,52 \%) lokalisiert. Bei fünf Patienten blieb der Primärtumor unbekannt (7,57 \%).

Tabelle 11 fasst die Verteilung der Lokalisation der Primärtumoren und deren Häufigkeit im Patientenkollektiv dieser Analyse zusammen.

Tabelle 11: Verteilung und Häufigkeit der Lokalisation der Primärtumoren unter den 66 Patienten dieser Analyse

\begin{tabular}{|c|c|c|}
\hline Primärtumor & Anzahl der Patienten & Anzahl der Patienten in \% \\
\hline Dünndarm & 25 & 37,88 \\
\hline Kolon & 6 & 9,06 \\
\hline Appendix & 3 & 4,45 \\
\hline Pankreas & 16 & 24,24 \\
\hline Lunge & 4 & 6,06 \\
\hline Ovar & 2 & 3,03 \\
\hline Primärtumor & Anzahl der Patienten & Anzahl der Patienten in \% \\
\hline $\begin{array}{l}\text { Extraadrenales abdo- } \\
\text { minelles Paragangliom }\end{array}$ & 1 & 1,52 \\
\hline Dünndarm + Appendix & 2 & 3,03 \\
\hline Lunge + Magen & 1 & 1,52 \\
\hline
\end{tabular}




\begin{tabular}{|l|c|c|}
\hline Dünndarm + Pankreas & 1 & 1,52 \\
\hline Unbekannt & 5 & 7,57 \\
\hline
\end{tabular}

\subsubsection{Grading bei Erstdiagnose}

Im Rahmen der Initialen Diagnostik konnten bei zehn Patienten $(15,15 \%)$ ein G1-Tumor, bei 17 Patienten (25,76\%) ein G2-Tumor und bei sechs Patienten (9,09\%) ein G3-Tumor nachgewiesen werden. Für die Hälfte der Patienten (33 Fälle, 50 \%) fehlen diesbezüglich die Daten.

\subsubsection{Staging bei Erstdiagnose}

Bei Erstdiagnose befand sich der Tumor bei drei Patienten im T1-Stadium (4,54\%), bei vier Patienten im T2-Stadium (6,06 \%) und bei zwei Patienten im T3-Stadium (3,03 \%). Bei 45 Patienten lag bei Erstdiagnose bereits ein T4-Stadium vor $(68,18 \%)$. Bei zwölf Patienten ist das Staging bei Erstdiagnose nicht bekannt (18,18\%).

Patienten, bei denen ein T4-Stadium vorlag, wiesen außerdem zum Zeitpunkt der Erstdiagnose bereits Metastasen auf $(68,18 \%)$. Somit ergibt sich neben dem Kollektiv der bereits metastasierten Patienten ein Kollektiv von 21 Patienten, die bei Erstdiagnose nicht metastasiert waren $(31,82 \%)$. Von den 45 Patienten mit bereits metastasierten Tumoren wiesen 43 hepatische Metastasen auf $(65,15 \%)$. Bei einem Patienten befanden sich die Metastasen zum Zeitpunkt der Erstdiagnose in der Lunge (1,52\%). Ein weiterer Patient zeigte zum Zeitpunkt der Erstdiagnose eine Peritonealkarzinose (1,52\%).

Tabelle 12 fasst die Verteilung des bei Erstdiagnose festgestellten Stagings zusammen.

Tabelle 12: Verteilung des bei Erstdiagnose festgestellten Stagings unter den 66 Patienten unseres Kollektivs

\begin{tabular}{|l|c|c|}
\hline \multicolumn{1}{|c|}{ Stadium } & Anzahl der Patienten & Prozentualer Anteil am Patientenkollektiv \\
\hline T1 & 3 & 4,54 \\
\hline T2 & 4 & 6,06 \\
\hline T3 & 2 & 3,03 \\
\hline T4 & 45 & 68,18 \\
\hline Nicht bekannt & 12 & 18,18 \\
\hline
\end{tabular}

\subsubsection{Funktionelle / nicht funktionelle Tumoren}

Zur Definition funktioneller und nicht-funktioneller Tumoren sei auf Kapitel 1.1.2 verweisen. Von den 66 beobachteten Patienten wiesen 45 einen funktionellen Tumor auf (68,18\%). Bei 21 Patienten lag ein nicht-funktioneller Tumor vor (31,82 \%). Zu Beginn der Therapie mit 
SAA ergab sich somit folgende Symptomatik im Gesamtkollektiv: 28 Patienten zeigten keine Karzinoidsymptomatik (42,42\%). Von den 38 Patienten, die eine Karzinoidsymptomatik zeigten $(57,8 \%)$, litten neun Patienten unter Flush $(13,64 \%)$ und vier Patienten unter Diarrhö (6,06 \%). 25 Patienten zeigten eine Symptomatik von Flush und Diarrhö (37,88 \%).

Bezogen auf die 45 Patienten, die einen funktionellen Tumor aufwiesen, ergibt sich folgende prozentuale Verteilung der Symptomatik: Sieben Patienten waren bezüglich der Karzinoidsymptomatik zum Zeitpunkt des Therapiebeginns mit SAA beschwerdefrei (15,56\%). Unter den 38 Patienten, die über eine Karzinoidsymptomatik klagten $(84,44 \%)$ zeigten neun eine Flush-Symptomatik (20\%), vier Patienten litten unter Diarrhöen $(8,89 \%)$ und 25 Patienten gaben sowohl Flush-Symptomatik, als auch Diarrhöen an $(55,56 \%)$.

\subsubsection{Sonstige Therapien}

Bei 42 Patienten $(63,64 \%)$ wurde der Tumor primär operativ behandelt. Davon wurde bei 25 Patienten (59,52\%) eine Teilresektion des betroffenen Organs vorgenommen, bei 17 Patienten $(40,48 \%)$ wurde hingegen eine radikale Resektion durchgeführt.

\section{Zeitraum ohne SAA}

Bei 16 Patienten (24,16\%) war schon vor der Therapie mit SAA eine Behandlung vorgenommen worden: 13 Patienten $(19,7 \%)$ erhielten vor der Therapie mit SAA eine Chemotherapie, sieben Patienten (10,6\%) wurden vor der Therapie mit SAA mit Interferon- $\alpha$ therapiert. Unter diesen Patienten befinden sich auch solche, die vor der Therapie mit SAA sowohl eine Chemotherapie, als auch eine Therapie mit Interferon- $\alpha$ bekommen hatten (4 Patienten). 50 Patienten $(75,5 \%)$ hatten vor der Therapie mit SAA keine andere Therapie erhalten. Nach Absetzten der SAA-Therapie fand bei 18 Patienten (27,18\%) eine weitere Therapie statt: drei Patienten $(4,54 \%)$ wurden nuklearmedizinisch mittels $Y^{90}$ DOTATOC therapiert, 17 Patienten $(25,67 \%)$ wurden weiterhin medikamentös behandelt, was in diesem Falle eine Chemotherapie, eine Therapie mit Interferon- $\alpha$ oder eine Kombination aus diesen beiden Therapien beinhaltete. Davon erhielten 11 Patienten $(64,7 \%)$ eine Chemotherapie, drei Patienten $(17,65$ $\%)$ wurden mit Interferon- $\alpha$ therapiert, bei drei Patienten $(14,65 \%)$ wurde sowohl eine Chemotherapie als auch eine Therapie mit Interferon- $\alpha$ durchgeführt. Unter oben aufgeführten Patienten befinden sich auch zwei, die sowohl eine nuklearmedizinische als auch eine medikamentöse Therapie erhalten hatten. 48 Patienten $(72,48 \%)$ blieben nach Absetzen der SAA ohne weitere Therapie. 
Hierbei sei zu erwähnen, dass beide Gruppen auch Patienten enthalten, die eine Kombination aus mehreren Therapien bekamen. Die Absoluten Zahlen entsprechen also nicht der Summe der Anzahlen in den Untergruppen (genaue absolute Zahlen siehe Abbildung 5).

\section{Zeitraum mit SAA}

Bei 29 Patienten wurde zeitgleich zu SAA noch eine weitere Therapie durchgeführt $(43,94$ \%). Jeder dieser Patienten erhielt eine medikamentöse Therapie, egal, welcher Art: 19 Patienten erhielten eine Chemotherapie $(65,52 \%)$, bei drei Patienten wurde eine Therapie mit Interferon- $\alpha$ durchgeführt (10,34\%), sieben Patienten erhielten sowohl eine Chemotherapie als auch eine Therapie mit Interferon- $\alpha(24,14 \%)$. Bei vier Patienten wurde zusätzlich zu dieser zeitgleichen medikamentösen Therapie auch eine nuklearmedizinische Therapie mittels $\mathrm{Y}^{90}$ DOTATOC durchgeführt $(13,8 \%)$. Somit ergibt sich eine verbleibende Zahl von 37 Patienten, bei der in diesem Zeitraum SAA als einzige Therapie eingesetzt wurde $(56,06 \%)$.

Abbildung 5 und 6 visualisieren die Einteilung in die jeweiligen Untergruppen bezüglich der Behandlungen.

Abbildung 7 gibt einen Überblick über die verschiedenen Therapien, die die Patienten zusätzlich zu SAA bzw. vor und nach der Therapie mit SAA erhalten haben. Dabei zeichnen die Zeiten „vor SAA“ und „nach SAA“ den oben genannten „Zeitraum ohne SAA“ nach, wobei sich die Angaben der Zeit „während SAA“ auf den oben genannten „Zeitraum mit SAA“ bezieht. 


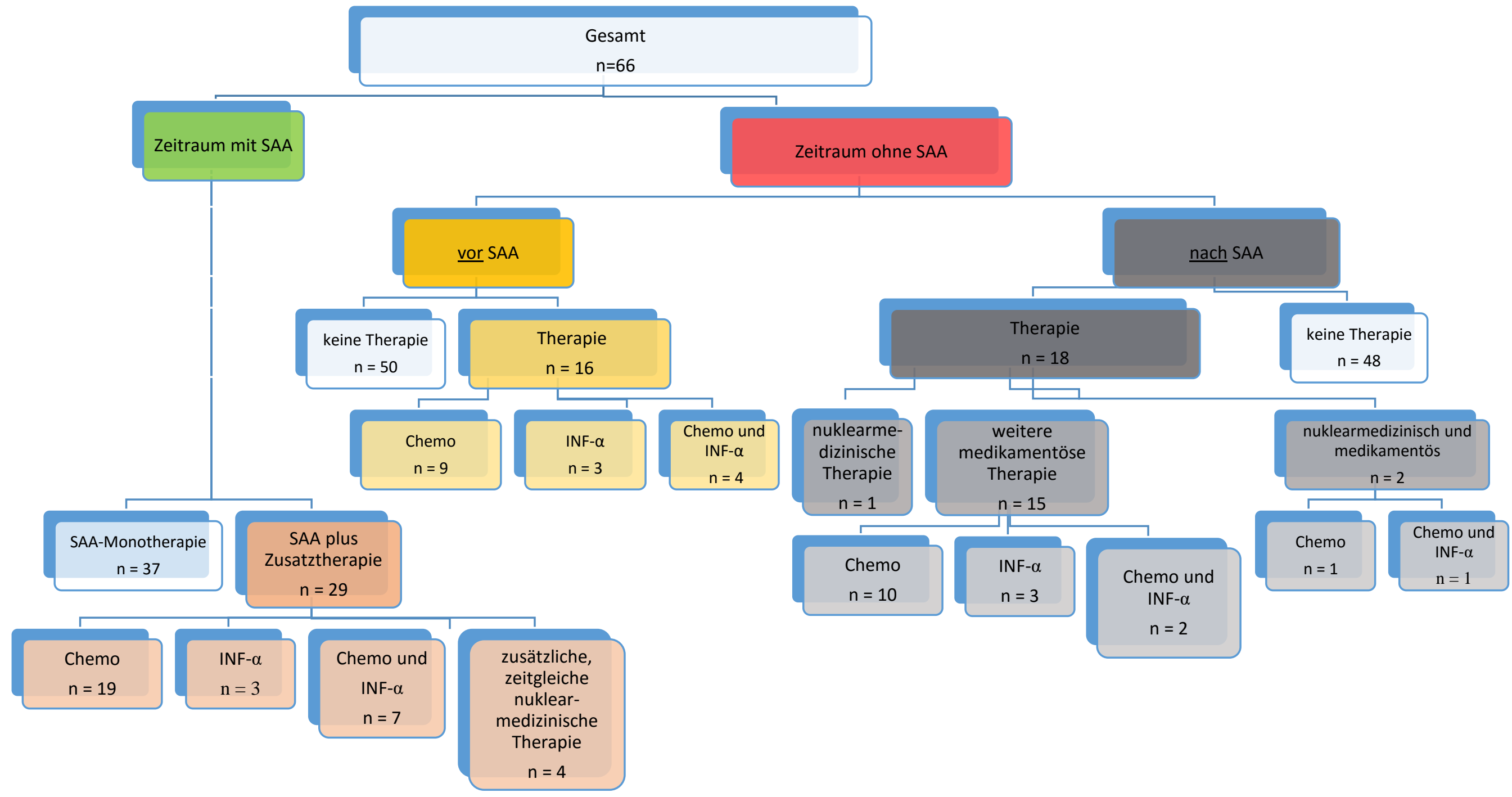

Abbildung 5: Baumdiagramm der Einteilung in die Zeitintervalle bezüglich durchgeführter Therapien im Zeitraum mit und ohne SAA beziehungsweise vor und nach einer Therapie mit SAA 


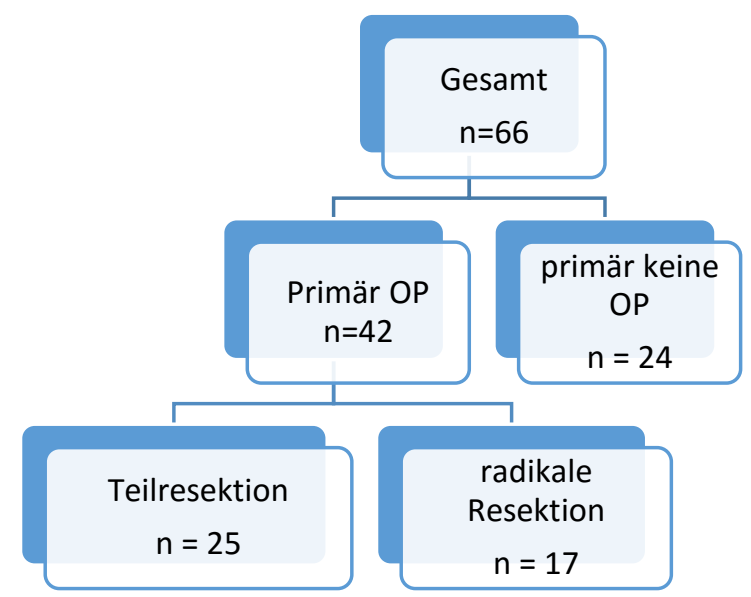

Abbildung 6: Baumdiagramm der Gruppeneinteilung nach operativer Therapie

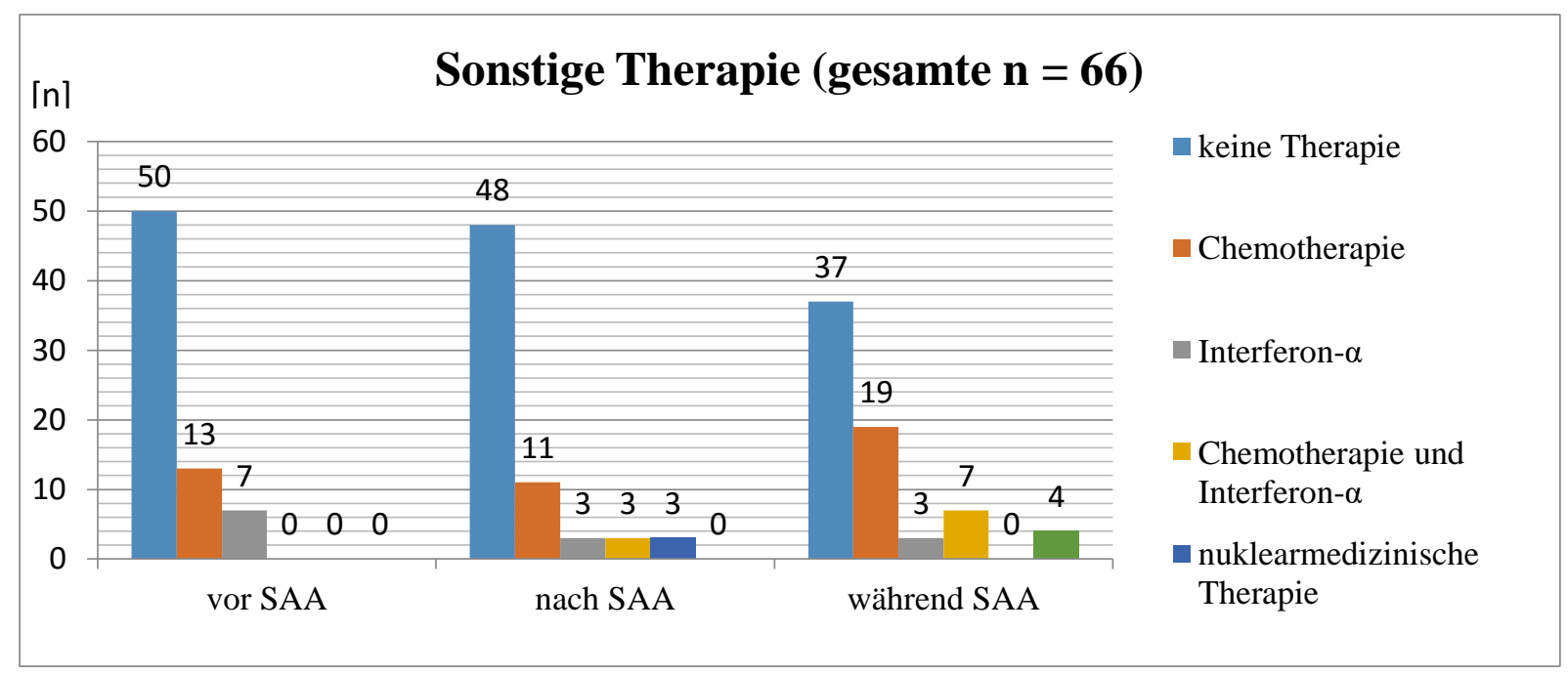

Abbildung 7: sonstige Therapien vor, nach und während der Therapie mit SAA in absoluter Anzahl der Patienten unseres Kollektivs $[n=66]$

Nach der weiteren Aufteilung in eine Gruppe mit SAA-Monotherapie und SAA plus Zusatztherapie im Zeitraum mit SAA ergibt sich folgende Patientenverteilung: 37 Patienten $(56,06 \%)$ erhielten eine SAA-Monotherapie. Davon blieben im Zeitraum ohne SAA 22 Patienten $(59,46 \%)$ ohne jegliche Therapie wohingegen bei 15 Patienten (40,54\%) eine alternative Therapie durchgeführt wurde. Unter den 29 Patienten $(43,94 \%)$, die im Zeitraum mit SAA eine zusätzliche Therapie erhalten hatten, lag die Zahl derer, die im Zeitraum ohne SAA ohne jegliche Therapie blieben, bei 18 (62,07 \%) und die Anzahl der Patienten mit alternativer Therapie betrug elf $(37,93 \%)$.

\subsection{Beobachtungsdauer}

Die mittlere Beobachtungsdauer betrug 102,62 \pm 84,59 Monate. Die kürzeste Beobachtungsdauer lag bei 3 Monaten, die längste bei 370 Monaten, was in Abbildung 8 dargestellt ist. 


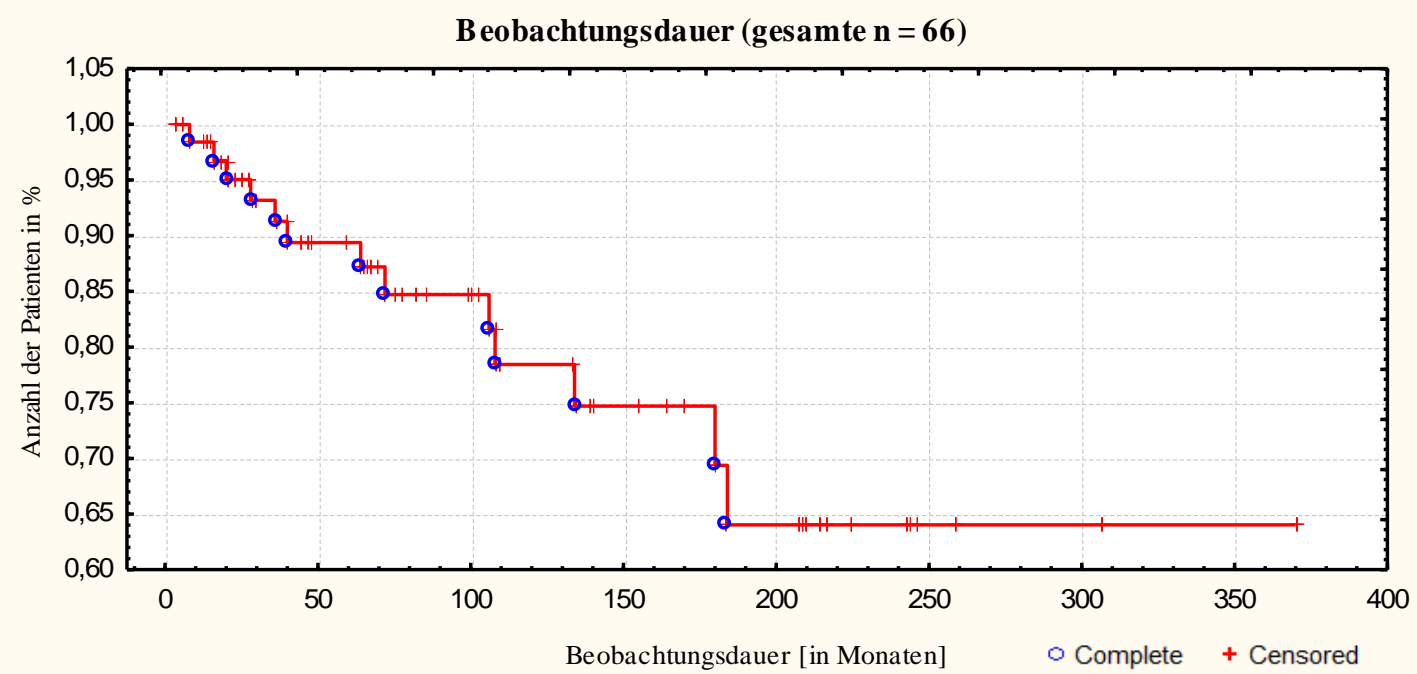

Abbildung 8: Kaplan-Meier Kurve über die Beobachtungsdauer als prozentueller Anteil der beobachteten Pateinten $(n=66)$. Unter "Complete" werden die in dieser Analyse während des Beobachtungszeitraums verstorbenen Patienten aufgeführt. Mit "Censored" werden Patienten bezeichnet, deren weitere Beobachtung nicht möglich war, da das Ende der Analyse erreicht war oder da sie aus der Analyse ausgeschieden waren (vergleiche Kapitel 3.2.2).

\subsubsection{Aus der Analyse ausgeschiedene Patienten}

39 Patienten (59 \%) schieden unter der Therapie mit SAA aus der Analyse aus. 13 davon waren verstorben $(20 \%)$, bei 28 war das weitere Verfolgen der Therapie nicht möglich (42\%). Von den 13 verstorbenen Patienten lag die Todesursache bei 12 Patienten an einer Tumorkachexie und einem damit verbundenen Herz-Kreislauf-Versagen. Bei einer Patientin kam es durch die wie in Kapitel 1.1.2 beschriebenen Effekte vasoaktiver Stoffe zu einem portalen Hypertonus und einem daraus resultierendem Rechtsherzversagen.

Gründe dafür, dass ein weiteres Verfolgen der Therapie nicht möglich war, können folgende sein:

- Patienten legten ihre weitere Behandlung aus Gründen der zu weiten Distanz zur Universitätsmedizin Göttingen in die Hände einer anderen Klinik

- Patienten waren aus Gründen der Betreuung durch Angehörige verzogen

- Patienten entschieden sich aus eigenem Willen gegen eine Fortsetzung der Therapie

\subsection{Therapie mit SAA}

In den folgenden Kapiteln werden jeweils Zeiträume, nicht notwendigerweise Patienten (beispielsweise „mit SAA“ vs. „ohne SAA“) miteinander verglichen. Bei den jeweiligen Ergebnissen ist daher zu beachten, dass unter Umständen. mehrere Beobachtungsintervalle pro Patient in die Analyse eingegangen sind. Hier beschriebene Verläufe beziehen sich auf den gesamten Zeitraum, in dem der jeweilige Patient mit SAA behandelt wurde, bzw. auf den ge- 
samten Zeitraum, in dem eine solche Therapie nicht stattfand. Der hierfür angegebene Beobachtungszeitraum ist in Kapitel 3.2.1 dargestellt. Betrachtet wird jeweils die gesamte Dauer der Beobachtung (also von Erstdiagnose bis zum Tod beziehungsweise. bis zum Ausscheiden aus der Analyse).

\subsubsection{Therapiedauer der SAA-Monotherapie}

Die durchschnittliche Dauer der Monotherapie mit SAA betrug 864,95 \pm 1404,14 Tagen. Das Minimum lag bei einem Tag, das Maximum bei 6390 Tagen.

In Monaten ergibt dies eine durchschnittliche Dauer der Monotherapie von 28,83 \pm 46,82 Monaten mit einem Minimum von 0 Monaten und einem Maximum von 213 Monaten (= 17,75 Jahren). Neun Patienten, die eine Dauer von null Monaten aufweisen, wurden lediglich zwischen einem und elf Tagen mit einer solchen Monotherapie behandelt.

Abbildung 9 stellt die Dauer der Monotherapie mit SAA noch einmal grafisch dar.

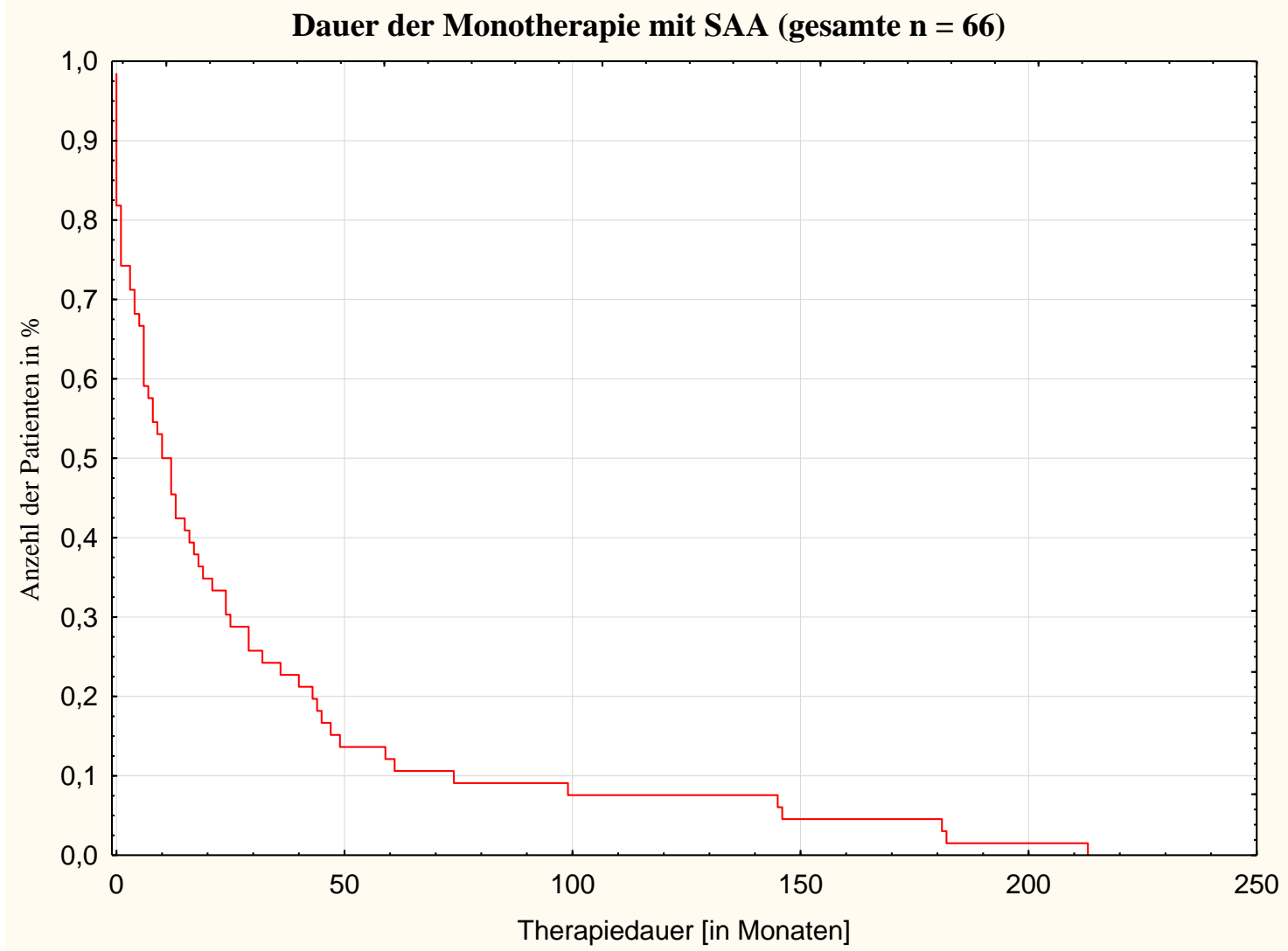

Abbildung 9: Kaplan-Meier-Kurve über die Dauer der Monotherapie mit SAA bezogen auf den prozentuellen Anteil der Patienten im Verlauf des Beobachtungszeitraums $(n=66)$

\subsubsection{Gesamtdauer der Therapie mit SAA}

Als Gesamtdauer der Therapie mit SAA wird der gesamte Zeitraum betrachtet, in dem der jeweilige Patient SAA erhalten hat. Es wird dabei nicht beachtet, ob und wenn ja welche $\mathrm{Zu}$ satztherapie zusätzlich eingesetzt wurde. 
In unserer Analyse konnte folgendes Verteilungsmuster dokumentiert werden: Die durchschnittliche Gesamtdauer der Therapie mit SAA betrug 47,68 \pm 50,84 Monate. Hierbei lag das Minimum bei 0 Monaten und das Maximum bei 212 Monaten. Eine Gesamtdauer von 0 Monaten hat dabei nicht zu bedeuten, dass eine solche Therapie nicht stattgefunden hat. Hierbei handelt es sich um eine Patientin, deren Gesamttherapiedauer lediglich 23 Tage betrug. Abbildung 10 stellt die Gesamtdauer der Therapie mit SAA grafisch dar.

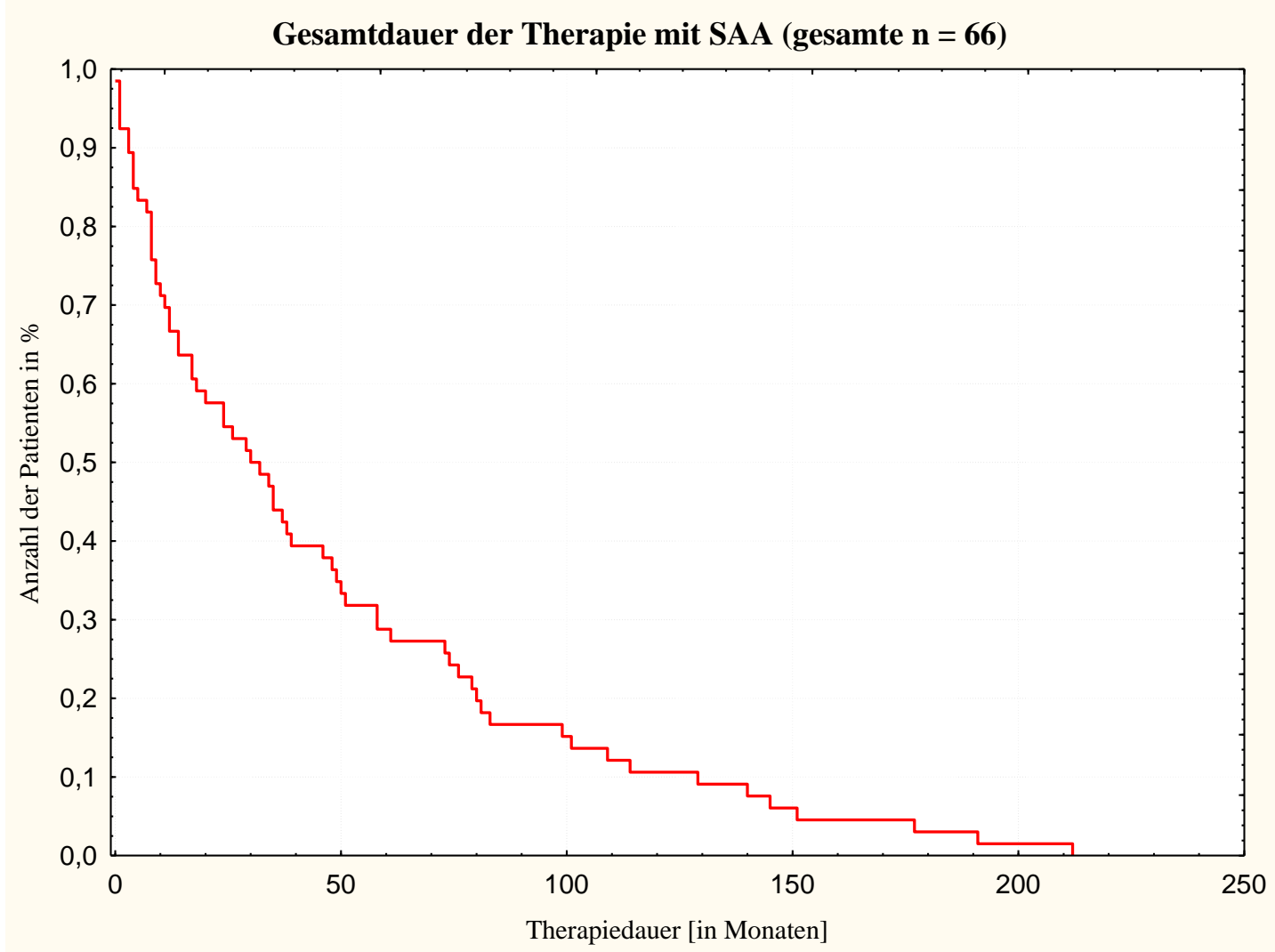

Abbildung 10: Kaplan-Meier-Kurve über die Gesamtdauer der Therapie mit SAA bezogen auf den prozentuellen Anteil der Patienten im Verlauf des Beobachtungszeitraums $(n=66)$

\subsubsection{Gesamtdauer der Zeitspanne ohne SAA}

Betrachtet man den Zeitraum, in dem die jeweiligen Patienten nicht mit SAA behandelt wurden, so ergab sich hierfür eine mittlere Zeitspanne von 58,17 \pm 66,41 Monate, wobei das Minimum bei einem Monat und das Maximum bei 301 Monaten lag.

Abbildung 11 stellt dies graphisch dar. 


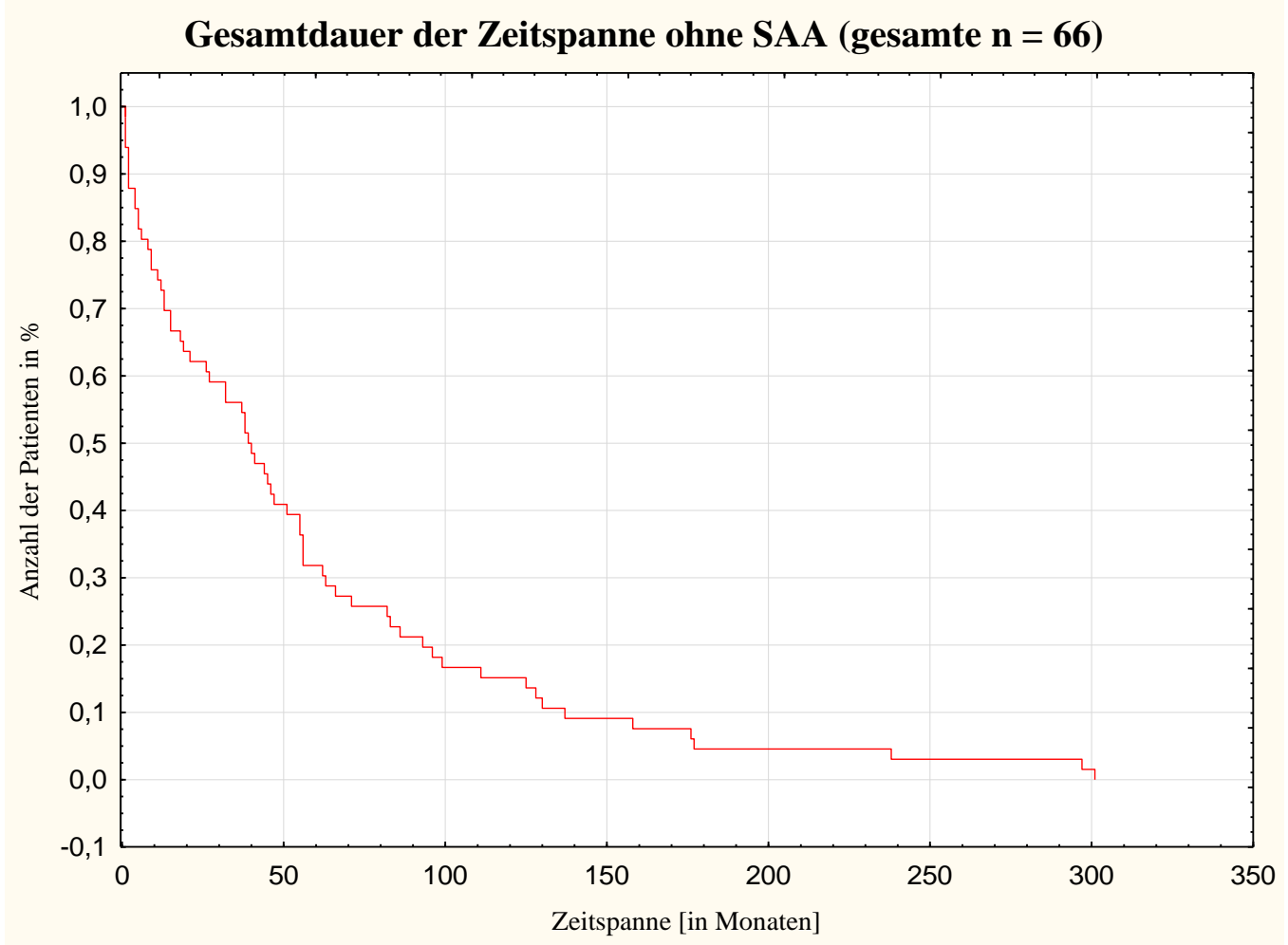

Abbildung 11: Kaplan-Meier-Kurve über die Gesamtdauer der Zeitspanne ohne SAA bezogen auf den prozentuellen Anteil der Patienten im Verlauf des Beobachtungszeitraums $(n=66)$

\subsubsection{Verlauf der Symptomatik}

Bezüglich der Datenerhebung für den Verlauf der Symptomatik und dessen Quantifizierung sei auf Kapitel 2.3.1 verwiesen.

Unter der Therapie mit SAA zeigten 28 Patienten keine Veränderung der Symptomatik $(42,28 \%)$. Bezogen auf die 45 Patienten mit funktionellen Tumoren ergibt sich folgende Verteilung: 33 Patienten zeigten unter SAA eine unveränderte Symptomatik (73,33 \%). Eine Zunahme der Symptomatik konnte bei vier Patienten beobachtet werden (8,88 \%). Acht Patienten konnten eine Abnahme der Symptomatik vorweisen (17,77 \%). Siehe Abbildung 12. 


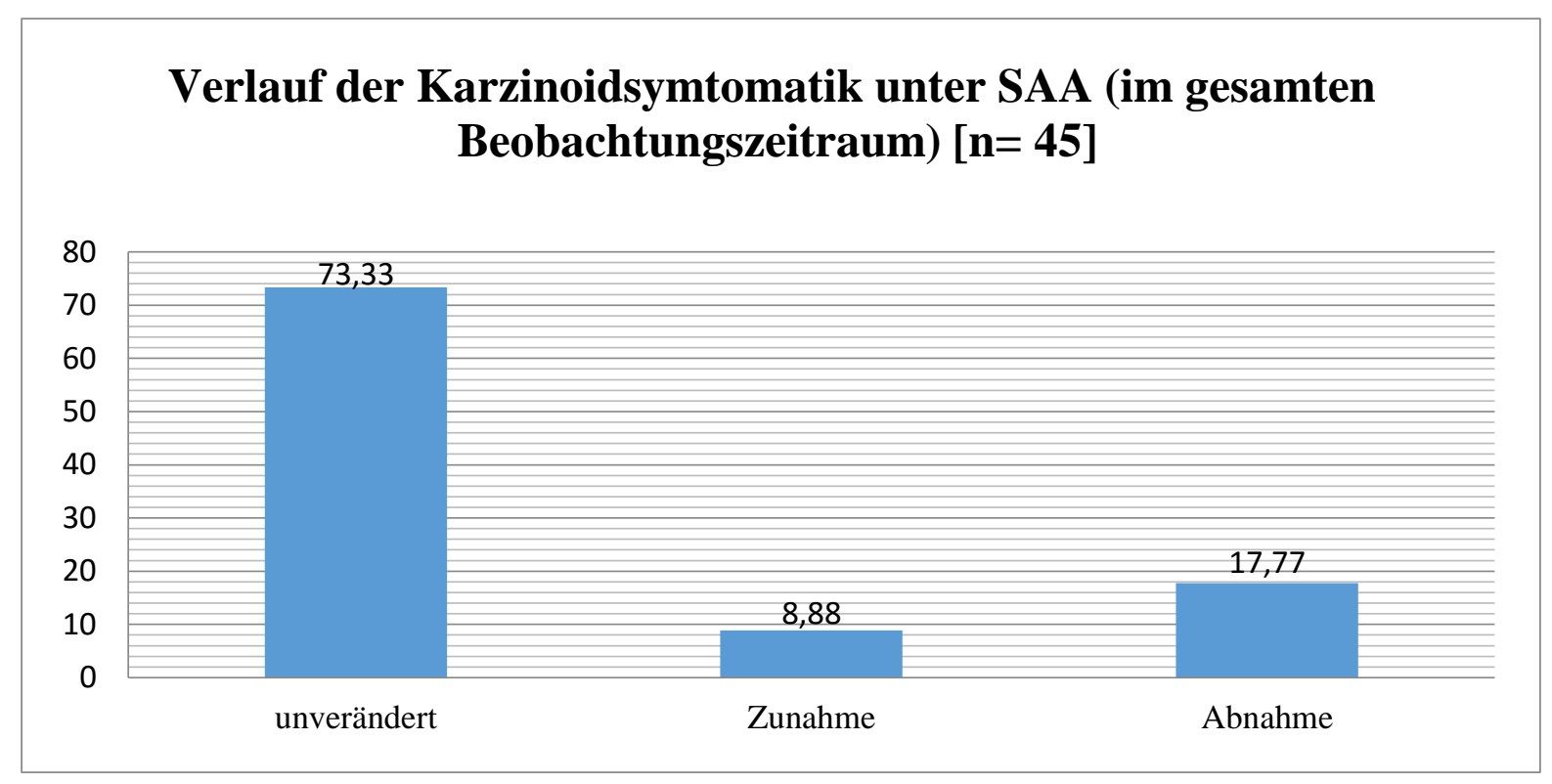

Abbildung 12: Verlauf der Karzinoidsymptomatik unter SAA bezogen auf die 45 Patienten mit funktionellem Tumor (im gesamten Beobachtungszeitraum)

[\%]

Bezogen auf die zu Therapiebeginn dokumentierte Symptomatik im gesamten Patientenkollektiv (funktionelle und nicht-funktionelle Tumor) konnte während der Therapie mit SAA folgende Verteilung beobachtet werden: Alle 28 Patienten, die zu Therapiebeginn bezüglich der Karzinoidsymptomatik beschwerdefrei gewesen waren, wiesen unter SAA diesbezüglich keine Veränderung auf $(42,42 \%)$. Bei den neun Patienten, die zu Therapiebeginn eine FlushSymptomatik gezeigt hatten, konnte ebenfalls keine Änderung beobachtet werden $(13,64 \%)$. Von den vier Patienten, die zu Therapiebeginn über Diarrhö geklagt hatten, blieb die Symptomatik bei einem Patienten unverändert (1,52 \%), zwei Patienten klagten über eine Zunahme (3,03\%), wohingegen ein Patient von einem Rückgang der Symptomatik berichten konnte $(1,52 \%)$. Unter den 25 Patienten, die zu Therapiebeginn über Flush und Diarrhö geklagt hatten, blieb die Symptomatik bei 16 Patienten unverändert (24,24 \%), zwei Patienten klagten über eine Zunahme (3,03\%), sieben Patienten berichteten über eine Abnahme (10,6\%). Die Prozentzahlen in Klammer beziehen sich hier jeweils auf das gesamte Patientenkollektiv, also auch auf die Patienten, die keine Karzinoidsymptomatik aufwiesen.

Der Verlauf der Karzinoidsymptomatik unter SAA ist in Abbildung 13 nach der zu Beginn der Therapie gezeigten Symptomatik aufgeschlüsselt und dargestellt. 


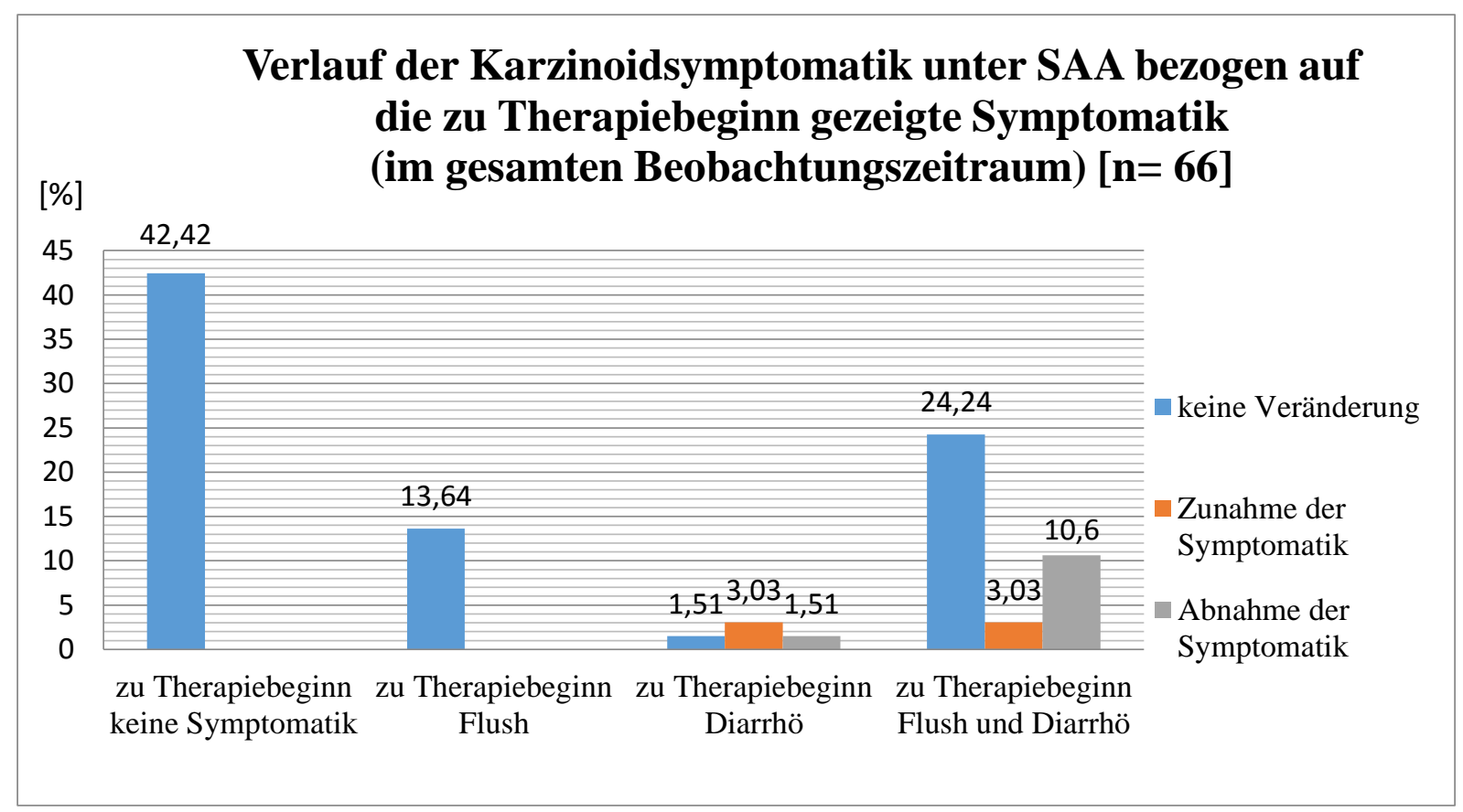

Abbildung 13: Verlauf der Karzinoidsymptomatik unter SAA bezogen auf alle 66 Patienten nach Symptomatik zu Therapiebeginn geordnet (im gesamten Beobachtungszeitraum)

\section{Monotherapie vs. Zusatztherapie}

Vergleicht man die Patientenkollektive „SAA-Monotherapie“ und „SAA plus Zusatztherapie“" innerhalb der Gesamtdauer der Therapie mit SAA wie sie in Kapitel 3.3.2 dargestellt ist, so ergibt sich folgende Verteilung: Unter den 37 Patienten mit SAA-Monotherapie zeigten 34 Patienten $(91,89 \%)$ eine unveränderte Symptomatik, bei keinem Patienten kam es zu einer Abnahme, bei drei Patienten $(8,11 \%)$ zu einer Zunahme der Beschwerden.

Im Patientenkollektiv „SAA plus Zusatztherapie“ zeigten 20 Patienten (68,96\%) eine unveränderte Symptomatik, vier Patienten (13,79\%) eine Zunahme und fünf Patienten (17,24 \%) eine Abnahme der Beschwerden.

Abbildung 14 stellt diese Verhältnisse graphisch dar. 


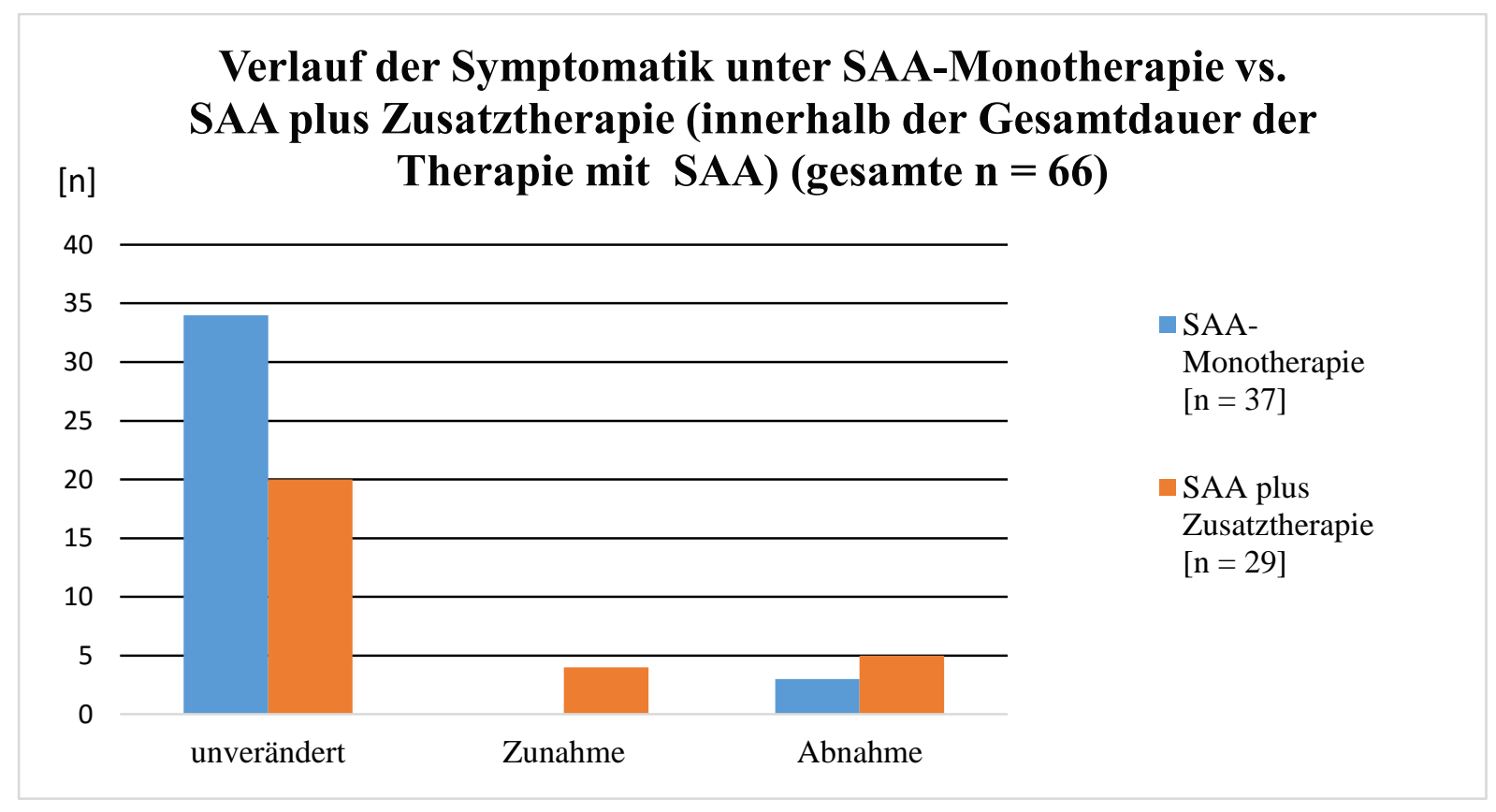

Abbildung 14: Verlauf der Symptomatik im Vergleich der 37 Patienten unter SAA Monotherapie mit den 29 Patienten unter SAA plus Zusatztherapie (innerhalb der Gesamtdauer der Therapie mit SAA)

\subsubsection{Verlauf der Tumormarker}

Leider liegt hier ein sehr heterogener und unvollständiger Datensatz bezüglich der Tumormarker vor. Es wird daher lediglich versucht, den Verlauf der Tumormarker nachzuzeichnen und einen eventuellen Therapieerfolg daraus abzuleiten. (Siehe auch Kapitel 4.3.3.) Die Einteilung in partielles Ansprechen, komplettes Ansprechen, kein Ansprechen und Progression erfolgt nach den in Kapitel 2.3.2 beschriebenen Kriterien.

Tumormarker bei Erstdiagnose

Bei Erstdiagnose konnte bei zehn Patienten (15,15\%) ein erhöhter Wert von CgA gemessen werden. Vier Patenten (6,06 \%) wiesen keine Erhöhung dieses Wertes auf. Von 52 Patienten $(78,79 \%)$ jedoch fehlen hier die Angaben. Bei 18 Patienten (27,27\%) konnten zu diesem Zeitpunkt erhöhte Werte für 5-HIAA im 24-Stunden-Urin festgestellt werden. Elf Patienten $(16,67 \%)$ wiesen ein normwertiges 5-HIAA auf, von 37 Patienten $(56,06 \%)$ fehlen die Angaben diesbezüglich. Für die Werte der NSE ergab sich eine Erhöhung bei fünf Patienten (7,56 \%), ein Normwert bei sechs Patienten $(9,09 \%)$ und eine fehlende Datenlage bei 55 Patienten $(83,33 \%)$. Ein erhöhtes Serotonin konnte bei 15 Patienten $(22,73 \%)$ gemessen werden, zwei Patienten $(3,03 \%)$ zeigten hierfür einen Normwert, für 49 Patienten $(74,24 \%)$ fehlen hier die Daten. 


\section{Tumormarker zum Zeitpunkt der Progression}

Bei Auftreten der Progression zeigten zwölf Patienten $(18,18 \%)$ einen erhöhten Wert für CgA. Bei vier Patienten $(6,06 \%)$ war dieser Wert nicht erhöht, von 50 Patienten $(75,76 \%)$ fehlen hierfür die Daten. Einen erhöhten Wert an 5-HIAA im 24-Stunden-Urin konnten 36 Patienten $(54,55 \%)$ aufweisen, bei neun Patienten $(13,64 \%)$ lag dieser Wert in der Norm, von 21 Patienten $(31,18 \%)$ fehlen die Daten diesbezüglich. Die Werte für NSE waren bei fünf Patienten $(7,56 \%)$ erhöht, bei drei Patienten $(4,55 \%)$ normwertig und bei 58 Patienten $(87,88 \%)$ den Daten nicht zu entnehmen. Serotonin war bei 27 Patienten (40,91 \%) erhöht und bei vier Patienten $(6,06 \%)$ normwertig, für 35 Patienten $(53,03 \%)$ fehlen diese Daten.

\section{Zeitraum ohne SAA}

Im Zeitraum ohne SAA wurde bei 30 Patienten kein Ansprechen beobachtet (45,45\%). Ein partielles Ansprechen konnte bei sieben Patienten beobachtet werden (10,6\%), drei Patienten erfuhren ein komplettes Ansprechen (4,54 \%). Eine Progression der Tumormarker trat bei 20 Patienten auf (30,3\%). Von sechs Patienten fehlen hier die Angaben $(9,09 \%)$.

\section{Zeitraum mit SAA}

Im Zeitraum mit SAA konnte bei 20 Patienten kein Ansprechen beobachtet werden (30,3 \%). Ein partielles Ansprechen wurde bei sechs Patienten beobachtet (9,09\%), weitere sechs Patienten erfuhren ein komplettes Ansprechen (9,09\%). Bei 28 Patienten trat eine Progression der Tumormarker auf (42,42\%). Auch hier fehlen die Angaben von sechs Patienten $(9,09 \%)$.

Abbildung 15 gibt einen Überblick über den Verlauf der Tumormarker im Zeitraum ohne SAA verglichen mit dem Zeitraum mit SAA.

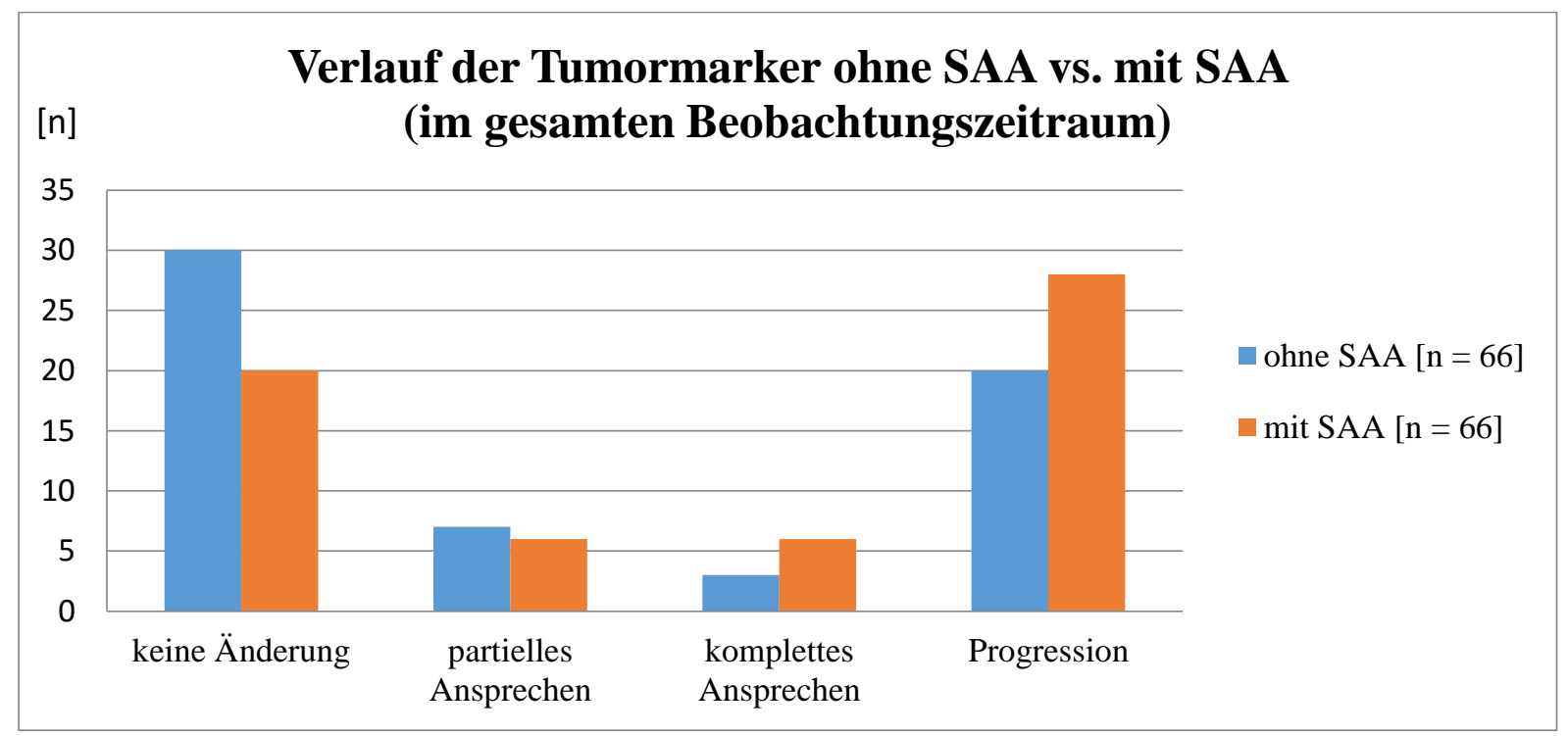

Abbildung 15: Verlauf der Tumormarker mit bzw. ohne SAA im Vergleich (im gesamten Beobachtungszeitraum) 
Wie schon in mehreren Studien nachgewiesen (Arnold et al. 1996; Garland et al. 2003; Wang et al. 2006), besteht keine Korrelation zwischen dem Verlauf der Symptomatik und dem Verlauf der Tumormarker. Vergleicht man Abbildung 14 und Abbildung 15, so wird dies auch anhand der hier aufgeführten Daten deutlich. (Beispiel: Zunahme der Symptomatik bei insgesamt 6,06 \% vs. Progression der Tumormarker bei 42,42\% der Patienten)

Ohne Zusatztherapie

Unter den 37 Patienten, bei denen während der Therapie mit SAA keine zusätzliche Therapie verabreicht wurde, war bei elf Patienten kein Ansprechen zu beobachten (29,73\%). Bei drei Patienten konnte ein partielles Ansprechen beobachtet werden $(8,11 \%)$, bei einem Patienten kam es zum kompletten Ansprechen (2,7\%). Eine Progression der Tumormarker trat bei 19 Patienten auf (51,35\%). Von drei Patienten fehlen hier die Angaben $(8,11 \%)$.

Im Zeitraum ohne SAA ergab sich im selben Patientenkollektiv bezüglich des Verlaufs der Tumormarker folgende Verteilung: Unter den 22 Patienten ohne jegliche Therapie kam es bei neun $(40,91 \%)$ zu keinem Ansprechen, bei vier Patienten (18,18\%) konnte ein partielles Ansprechen beobachtet werden. Keiner der Patienten dieser Gruppe zeigte ein komplettes Ansprechen. Fünf Patienten (22,73\%) erfuhren eine Progression der Werte. Von vier Patienten $(18,18 \%)$ fehlen hier die Daten. In der Gruppe der 15 Patienten, die eine alternative Therapie erhielten, zeigten sieben (46,67 \%) kein Ansprechen. Ein Patient (6,67 \%) erfuhr ein partielles Ansprechen wohingegen zwei Pateinten (13,34\%) ein komplettes Ansprechen erfuhren. Bei sieben Patienten $(33,34 \%)$ kam es zur Progression.

Abbildung 16 stellt die genannten Verhältnisse in der Gruppe der Patienten mit SAAMonotherapie dar. 


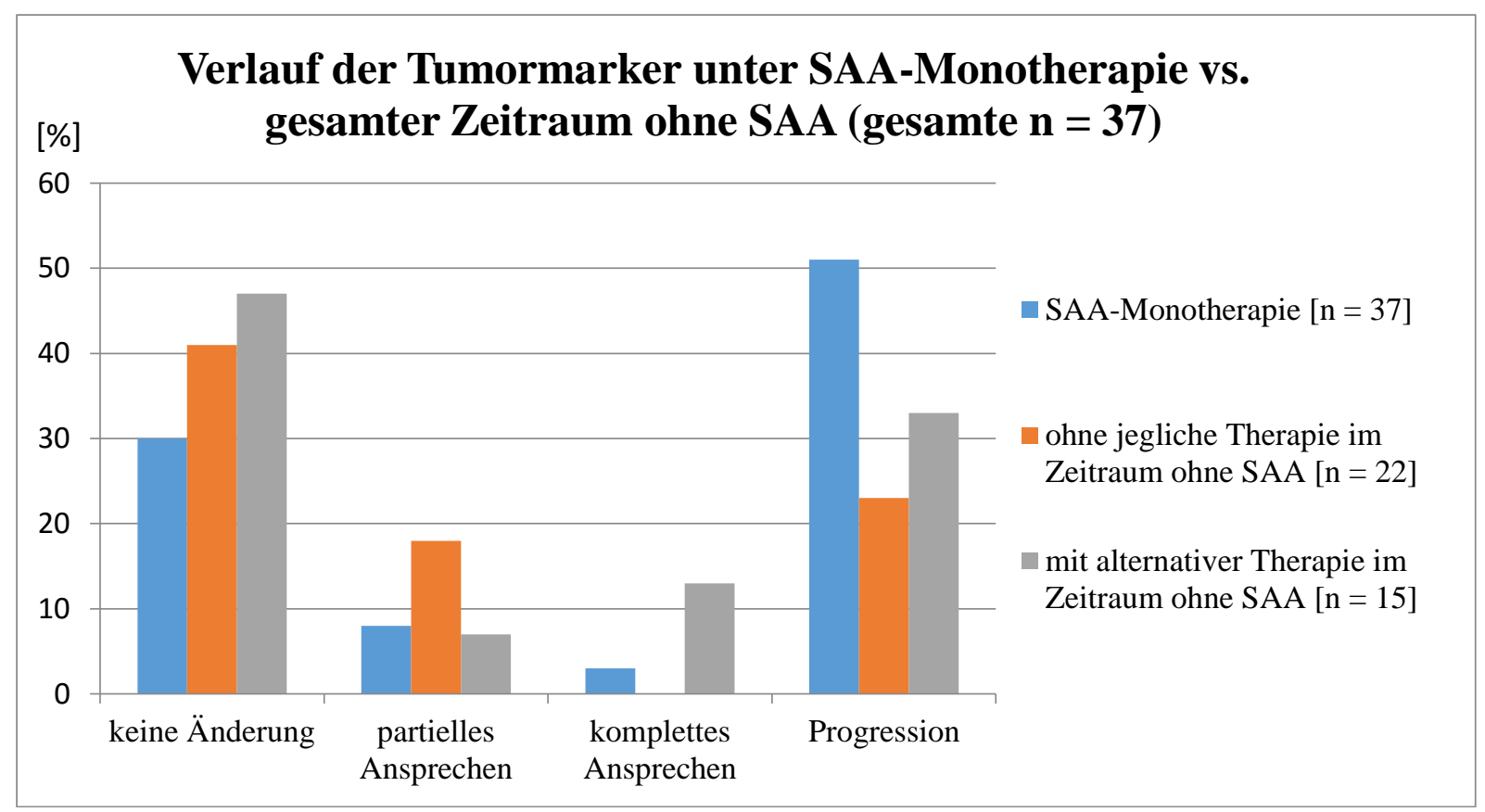

Abbildung 16: Verlauf der Tumormarker im Patientenkollektiv der 37 Patienten mit SAA-Monotherapie verglichen mit dem gesamten Zeitraum ohne SAA, unterteilt in die Gruppen "ohne jegliche Therapie" (22 Patienten) und "alternative Therapie" (15 Patienten)

\section{$\underline{\text { Mit Zusatztherapie }}$}

Unter den 29 Patienten, die während der Therapie mit SAA noch eine zusätzliche Therapie erhielten, konnte bei neun Patienten kein Ansprechen beobachtet werden (31,03\%). Bei drei Patienten wurde ein partielles Ansprechen beobachtet (10,34 \%), bei fünf Patienten kam es zum kompletten Ansprechen (17,24 \%). Bei neun Patienten trat eine Progression der Tumormarker auf (31,03\%). Hier fehlen die Angaben von drei Patienten (10,34\%).

Für den Zeitraum ohne SAA ergeben sich im selben Patientenkollektiv folgende Beobachtungen für den Verlauf der Tumormarker: Unter den 18 Patienten ohne jegliche Therapie kam es bei acht $(44,44 \%)$ zu keinem Ansprechen, ein Patient (5,55\%) zeigte ein partielles Ansprechen wohingegen ein komplettes Ansprechen bei keinem Patienten gesehen werden konnte. Eine Progression trat bei sieben Patienten $(38,89 \%)$ ein. Von zwei Patienten $(11,11 \%)$ fehlen hierfür die Daten. Von den elf Patienten, die eine alternative Therapie erhalten hatten, zeigten sechs (54,54\%) kein Ansprechen, ein Patient (9,09\%) ein partielles Ansprechen, ein weiterer Patient (9,09 \%) ein komplettes Ansprechen und drei Patienten (27,27\%) eine Progression.

Abbildung 17 verdeutlicht die oben genannten Verhältnisse in der Gruppe der Patienten mit Zusatztherapie. 


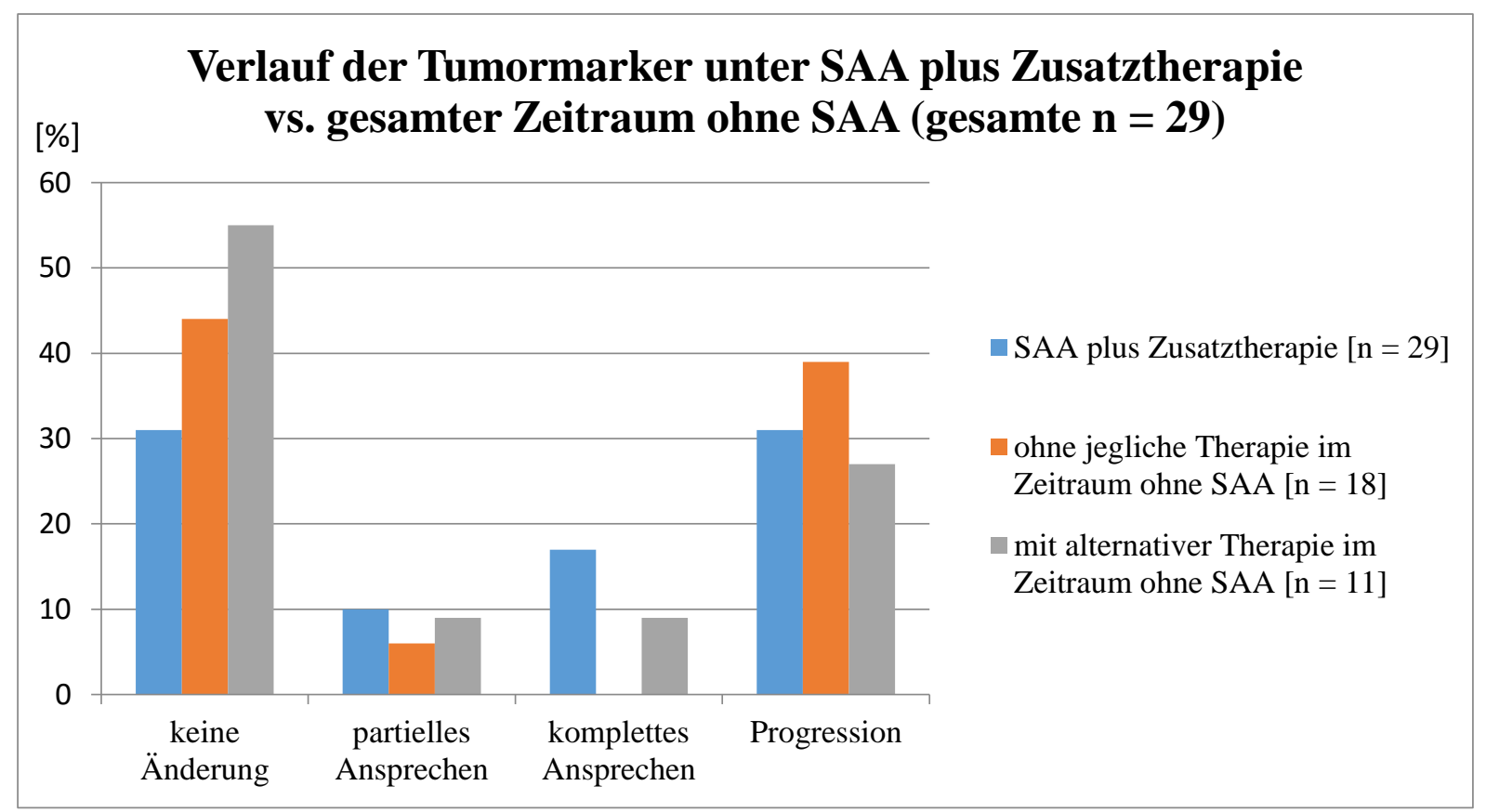

Abbildung 17: Verlauf der Tumormarker im Patientenkollektiv der 29 Patienten mit SAA plus Zusatztherapie verglichen mit dem gesamten Zeitraum ohne SAA, unterteilt in die Gruppen "ohne jegliche Therapie“ (18 Patienten) und "alternative Therapie" (11 Patienten)

In Abbildung 18 wird der Verlauf der Tumormarker unter SAA-Monotherapie mit dem Verlauf der Tumormarker unter SAA plus Zusatztherapie im gesamten Zeitraum der SAATherapie verglichen und dargestellt.

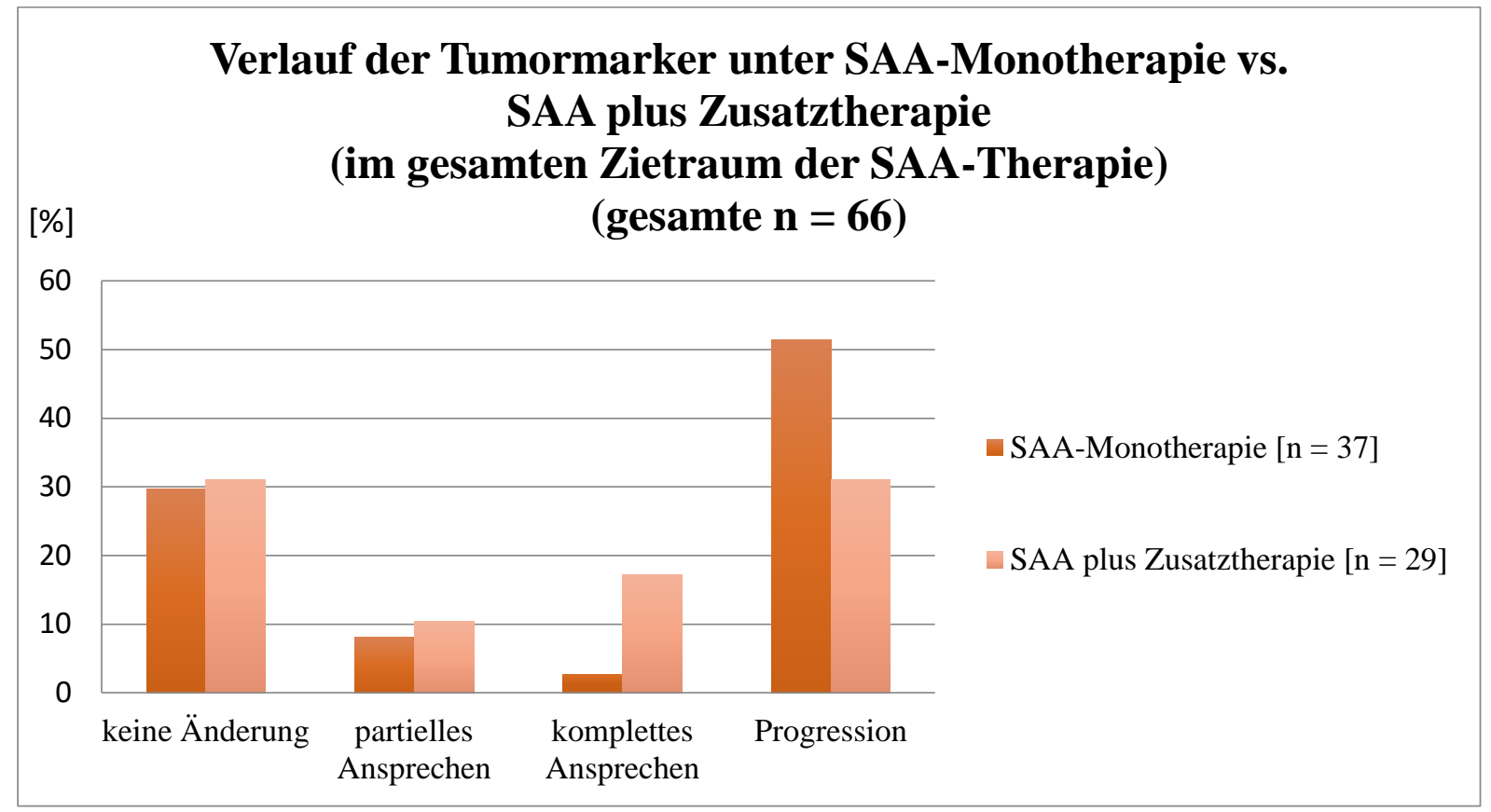

Abbildung 18: Verlauf der Tumormarker unter SAA. Verglichen werden die Verläufe der 37 Patienten mit SAA-Monotherapie mit den Verläufen der 29 Patienten, die eine zusätzliche Therapie erhalten haben im gesamten Zeitraum der SAA-Therapie 


\subsubsection{Stable disease unter SAA}

Eine stable disease, gemessen am Verlauf der Tumorgröße unter SAA dauerte durchschnittlich 21,44 $\pm 26,5$ Monate an. Das Minimum lag hier bei 0 Monaten, das Maximum bei 144 Monaten. Nach zwölf Monaten befanden sich $50 \%$ der Patienten nicht mehr in einer stable disease.

Abbildung 19 zeigt die Dauer der stable disease für alle Patienten im dokumentierten Zeitraum.

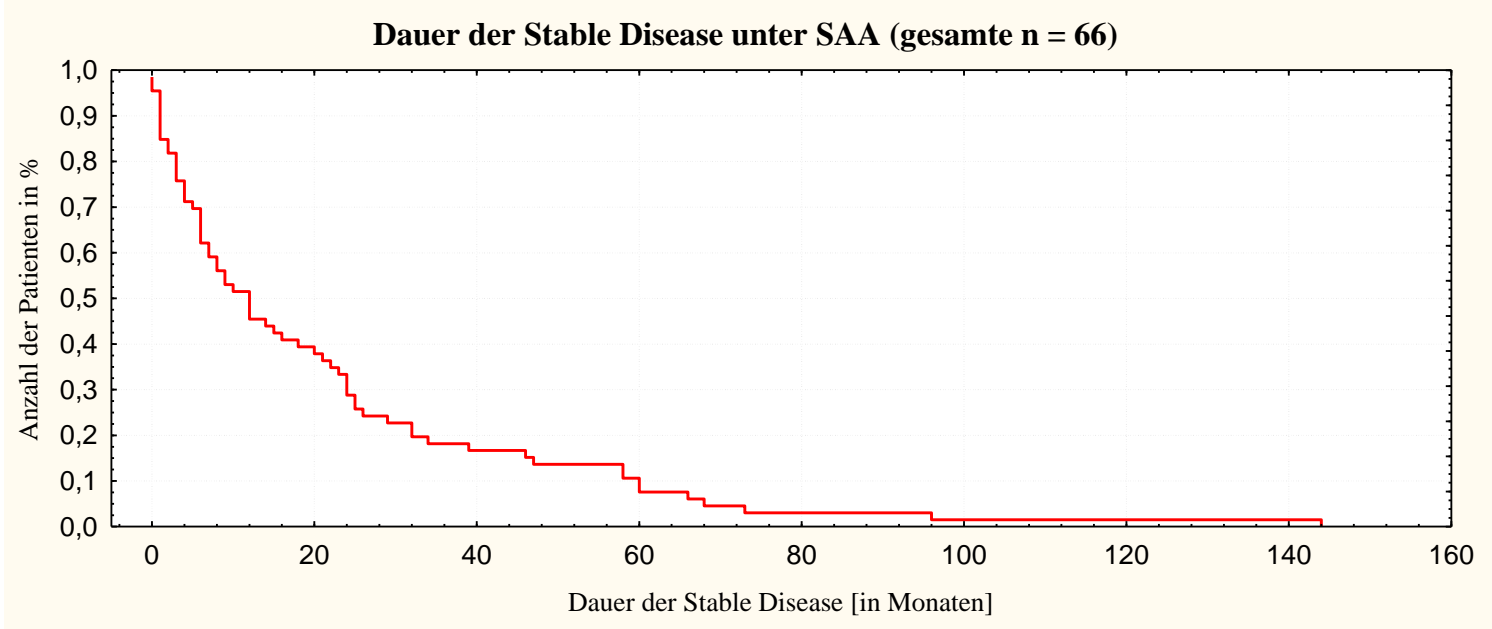

Abbildung 19: Kaplan-Meier-Kurve für die Dauer der stable disease unter der Therapie mit SAA gemessen am Verlauf der Tumorgröße im Beobachtungszeitraum $(n=66)$

\subsubsection{Dauer der stable disease nach Metastasierung bei Erstdiagnose geordnet}

Unterscheidet man während der Zeit der Therapie mit SAA in ein Patientenkollektiv, das bei Erstdiagnose bereits eine Metastasierung aufwies und eines, bei denen zum Zeitpunkt der Erstdiagnose keine Metastasen nachgewiesen werden konnten, so ergeben sich folgende Befunde: Patienten, die bei Erstdiagnose bereits eine Metastasierung aufwiesen hatten eine mittlere Dauer der stable disease von 23,02 \pm 24,38 Monaten. Hier lag das Minimum bei 0 Monaten und das Maximum bei 96 Monaten. Patienten, bei denen zum Zeitpunkt der Erstdiagnose noch keine Metastasen nachgewiesen werden konnten, hatten eine durchschnittliche Dauer der stable disease von 18,05 \pm 30,91 Monaten. Hier lag das Minimum bei 0 Monaten und das Maximum bei 144 Monaten. Nach neun Monaten befanden sich $50 \%$ der Patienten, die primär nicht metastasiert waren, nicht mehr in der stable disease. In der Gruppe der primär metastasierten betrug das Intervall bis zum Progress bei $50 \%$ der Patienten zwölf Monate.

Der Vergleich dieser beiden Patientenkollektive zeigte sich jedoch als statistisch nicht signifikant $(\mathrm{p}=0,48)$. 
Abbildungen 20 zeigt die Dauer der stable disease dieser Patientenkollektive im Vergleich.

Dauer der Stable Disease nach Metastasierung bei Erstdiagnose geordnet (gesamte $n=66$ )

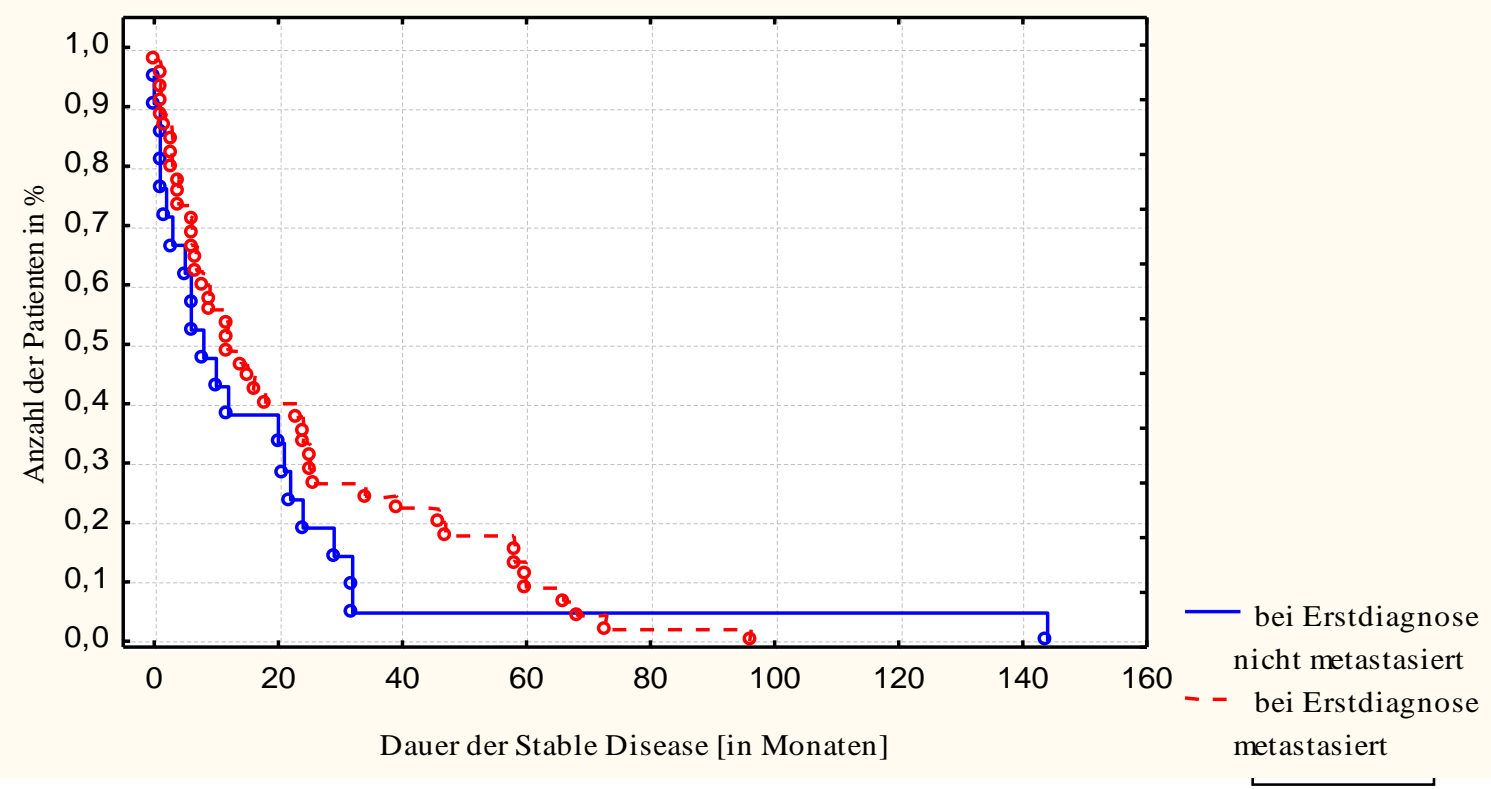

Abbildung 20: Kaplan-Meier-Kurve zur Dauer der stable disease unter SAA unter Berücksichtigung des Vergleichs nach primär metastasierten und primär nicht metastasierten Tumoren $(n=66)$. Signifikanzniveau mittels T-Test für unabhängige Gruppen: $p=0,48$.

\subsubsection{Dauer der stable disease nach Zusatztherapie geordnet}

Unterscheidet man im Zeitraum der Therapie mit SAA in ein Patientenkollektiv mit Zusatztherapie und eines ohne Zusatztherapie so ergeben sich folgende Werte: Patienten, die keine zusätzliche Therapie erhalten hatten, hatten eine durchschnittliche Dauer der stable disease von 21,19 $\pm 28,87$ Monaten mit einem Minimum von 0 Monaten und einem Maximum von 144 Monaten. Die mittlere Dauer der stable disease bei Patienten, die eine Zusatztherapie erhalten hatten, betrug 21,76 \pm 23,61 Monate. Das Minimum lag bei 0 Monaten und das Maximum bei 96 Monaten. Nach elf Monaten waren sowohl in der Gruppe mit, als auch in der Gruppe ohne Zusatztherapie $50 \%$ der Patienten nicht mehr in der stable disease.

Der Vergleich der beiden genannten Patientenkollektive erlangt jedoch keine statistische Signifikanz $(\mathrm{p}=0,93)$.

Abbildung 21 zeigt die Dauer der stable disease der beiden oben genannten Patientenkollektive im Vergleich. 


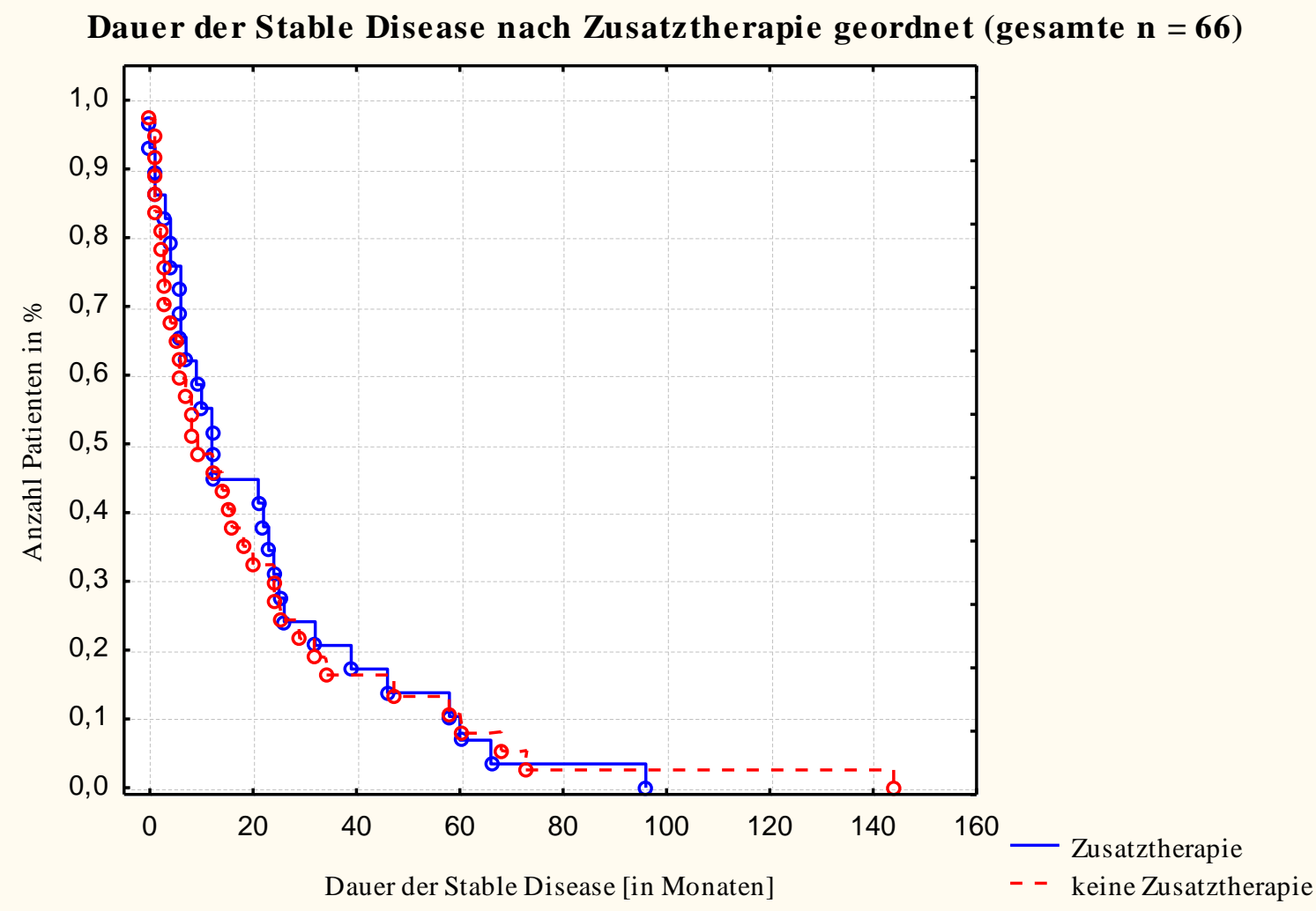

Abbildung 21: Kaplan-Meier-Kurve für die Dauer der stable disease unter SAA unter Berücksichtigung des Vergleichs von Patienten mit Zusatztherapie vs. ohne Zusatztherapie $(n=66)$. Signifikanzniveau mittels $t$-Test für unabhängige Gruppen: $p=0,93$.

\subsubsection{Verlauf der Tumorgröße}

Im Folgenden wird der Verlauf der Tumorgröße nach den RECIST-Kriterien (vgl. Kapitel 2.3.2) im Zeitraum mit SAA verglichen mit dem Zeitraum ohne SAA analysiert.

\section{Zeitraum ohne SAA}

Im Zeitraum ohne SAA war bei neun Patienten eine stable disease zu beobachten $(13,64 \%)$. Bei keinem der Patienten trat eine partial response auf, bei 48 Patienten konnte eine progressive disease beobachtet werden (72,73\%), ein Rezidiv des Primärtumors entwickelten neun Patienten (13,64\%). Von einem Patienten fehlen die Angaben über den genannten Zeitraum $(1,52 \%)$.

\section{Zeitraum mit SAA}

Im Zeitraum mit SAA wurde bei 31 Patienten eine stable disease beobachtet $(46,97 \%)$. Partial response trat bei einem Patienten auf (1,52\%), eine progressive disease konnte bei 33 Patienten beobachtet werden (50\%). Unter der Therapie mit SAA entwickelte keiner der Patienten ein Rezidiv. Auch hier fehlen die Angaben eines Patienten (1,52\%). 
Abbildung 22 fasst den Verlauf der Tumorgröße zusammen und vergleicht dabei zwischen dem Zeitraum mit und dem Zeitraum ohne SAA.

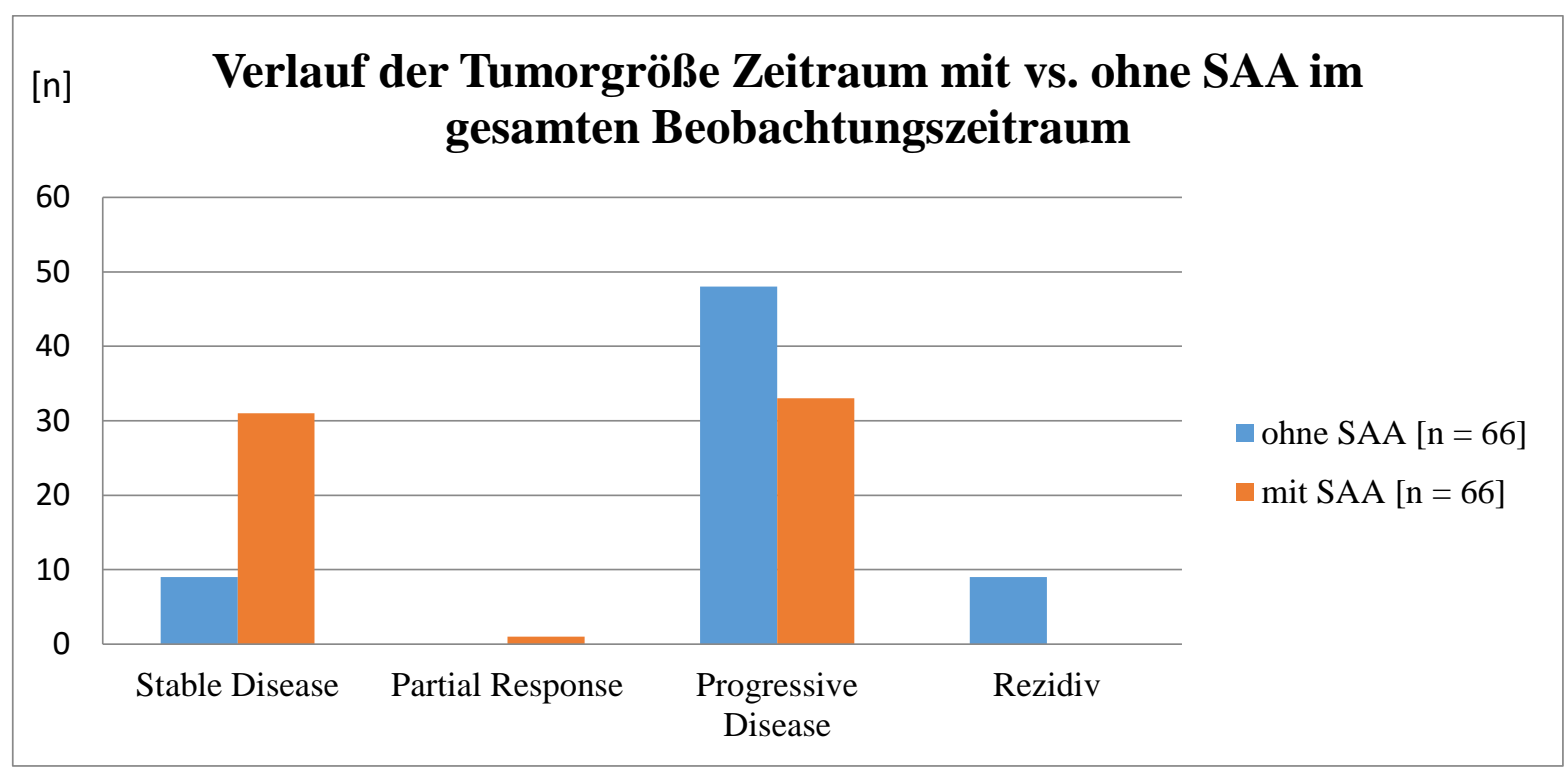

Abbildung 22: Verlauf der Tumorgröße mit bzw. ohne SAA während des gesamten Beobachtungszeitraums im Vergleich $(n=66)$

\section{Ohne Zusatztherapie}

Unter den 37 Patienten, bei denen eine SAA-Monotherapie durchgeführt wurde, zeigten 16 $(43,24 \%)$ eine stable disease. Bei einem Patienten $(2,7 \%)$ kam es zur partial response, wobei 19 Patienten $(51,35 \%)$ eine progressive disease erfuhren. Zu einem Rezidiv kam es bei keinem der Patienten. Von einem Patienten (2,7 \%) fehlen die Angaben.

Für den Zeitraum ohne SAA ergeben sich im selben Kollektiv folgende Verteilungen: Unter den 22 Patienten ohne jegliche Therapie kam es bei fünf Patienten $(22,73 \%)$ zu einer stable disease, bei keinem Patienten zur partial response, bei 14 Patienten $(63,64 \%)$ zur progressive disease und bei drei Patienten $(13,64 \%)$ zu einem Rezidiv. Unter den 15 Patienten mit alternativer Therapie konnte in keinem der Fälle eine stable disease oder eine partial response beobachtet werden. 13 Patienten $(86,67 \%)$ zeigten eine progressive disease und zwei Patienten $(13,34 \%)$ ein Rezidiv.

Abbildung 23 gibt einen Überblick über genannte Verhältnisse im Patientenkollektiv mit SAA-Monotherapie. 


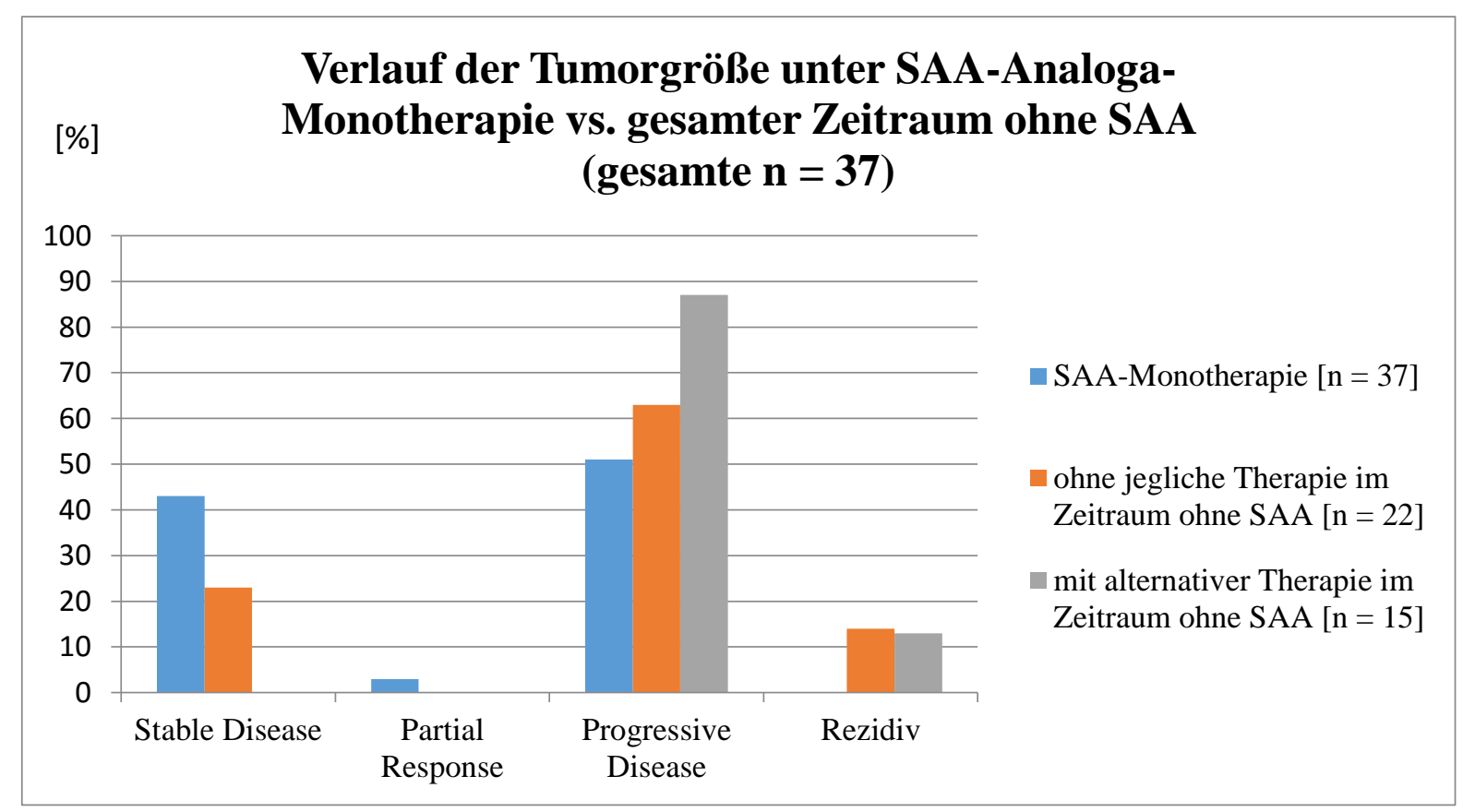

Abbildung 23: Verlauf der Tumorgröße im Kollektiv der 37 Patienten mit SAA-Monotherapie verglichen mit dem gesamten Zeitraum ohne SAA, unterteilt in die Gruppen "ohne jegliche Therapie“ (22 Patienten) und "alternative Therapie“ (15 Patienten)

\section{$\underline{\text { Mit Zusatztherapie }}$}

Unter den 29 Patienten, die eine zusätzliche Therapie erhielten ergaben sich für den Zeitraum mit SAA folgende Beobachtungen: Eine stable disease war bei 15 Patienten $(51,72 \%)$ und eine partial response bei keinem Patienten zu beobachten. Progressive disease trat bei 14 Patienten (48,28\%) auf. Keiner der Patienten entwickelte ein Rezidiv.

Im selben Kollektiv erfuhren im Zeitraum ohne SAA unter den 18 Patienten ohne jegliche Therapie drei Patienten (16,67 \%) eine stable disease, kein Patient eine partial response und 15 Patienten (83,34\%) eine progressive disease. Keiner dieser Patienten entwickelte ein Rezidiv. Bei den elf Patienten, die eine alternative Therapie erhalten hatten konnte bei einem Patienten $(9,09 \%)$ eine stable disease beobachtet werden. Keiner dieser Patienten wies eine partial response auf, sechs Patienten $(54,54 \%)$ erfuhren eine progressive disease und vier Patienten (36,36\%) entwickelten ein Rezidiv.

In Abbildung 24 sind oben genannte Verhältnisse für den Verlauf des Tumorwachstums veranschaulicht. 


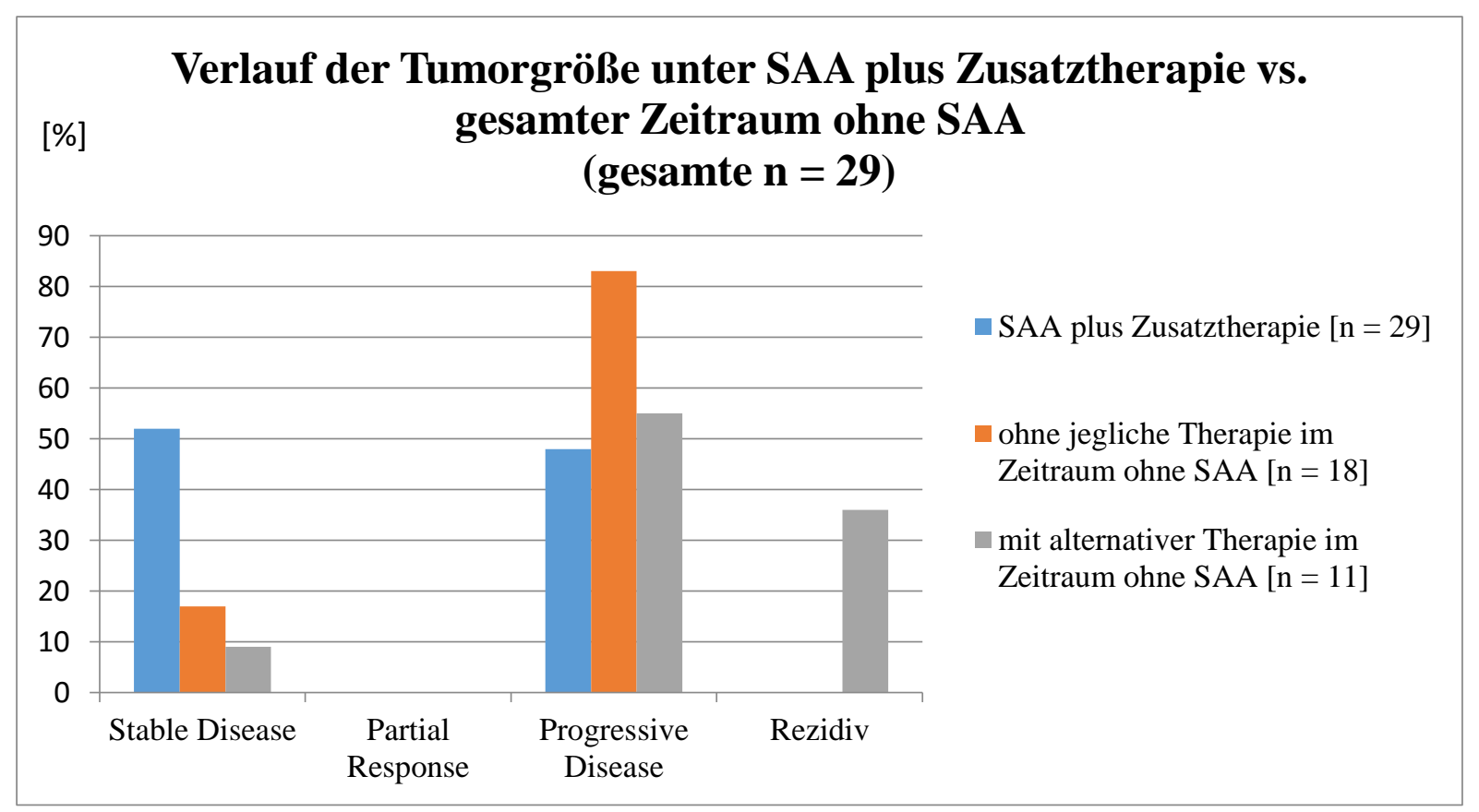

Abbildung 24: Verlauf der Tumorgröße im Kollektiv der 29 Patienten mit SAA plus Zusatztherapie verglichen mit dem gesamten Zeitraum ohne SAA, unterteilt in die Gruppen "ohne jegliche Therapie" (18 Patienten) und "alternative Therapie" (elf Patienten)

\subsubsection{Entstehung hepatischer Metastasen}

\section{Zeitraum ohne SAA}

Im Zeitraum ohne SAA traten bei zwölf Patienten neue Metastasen in der Leber auf $(18,18 \%)$. Ein Patient entwickelte ein Rezidiv einer bereits durch vorherige Therapie (Chemotherapie/INF- $\alpha$ ) komplett zurückgebildeten Metastase (1,52\%). Bei 53 Patienten waren in diesem Zeitraum keine neuen hepatischen Metastasen aufgetreten (80,3\%).

\section{Zeitraum mit SAA}

Während der Therapie mit SAA wurde bei fünf Patienten das Entstehen neuer hepatischer Metastasen beobachtet (7,57 \%). Keiner der Patienten entwickelte ein Rezidiv. Bei 61 Patienten konnte während dieses Zeitraums keine Entstehung hepatischer Metastasen nachgewiesen werden $(92,42 \%)$.

In Abbildung 25 wird die Entstehung hepatischer Metastasen im Zeitraum mit und im Zeitraum ohne SAA zusammengefasst und verglichen. 


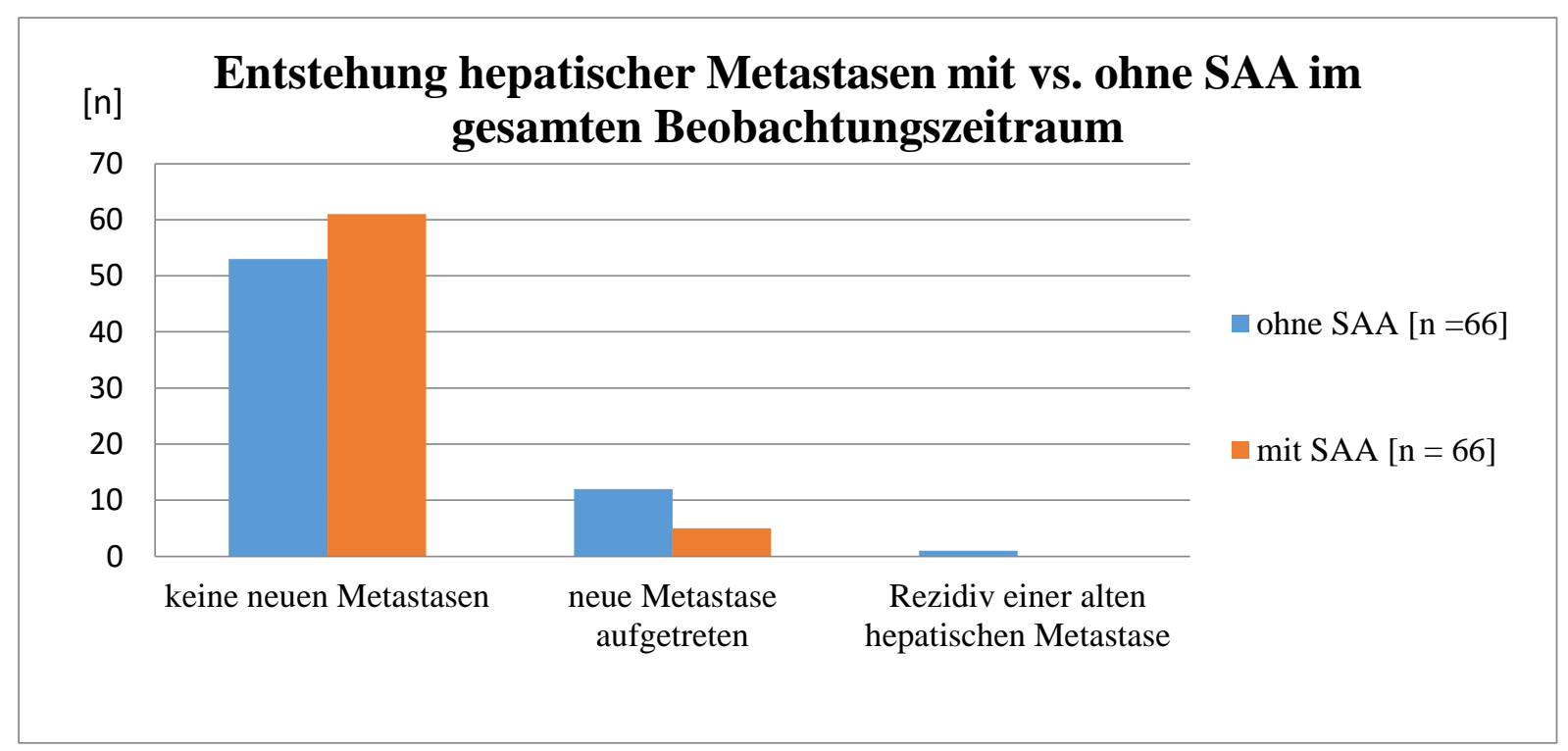

Abbildung 25: Entstehung hepatischer Metastasen mit bzw. ohne SAA im gesamten Beobachtungszeitraum im Vergleich $(n=66)$

Ohne Zusatztherapie

Im Patientenkollektiv der 37 Patienten unter SAA-Monotherapie traten bei 34 Patienten $(91,89 \%)$ keine neuen Metastasen auf, wobei ein Entstehen neuer Metastasen bei drei Patienten $(8,12 \%)$ beobachtet werden konnte. Bei keinem Patienten trat ein Rezidiv auf.

Im Zeitraum ohne SAA ergaben sich für dasselbe Patientenkollektiv folgende Verhältnisse: $14(63,64 \%)$ der 22 Patienten, die ohne jegliche Therapie blieben entwickelten keine neuen Metastasen, wobei bei acht Patienten (36,36 \%) neue Metastasen auftraten. Keiner dieser Patienten entwickelte ein Rezidiv. Unter den 15 Patienten, die mit einer alternativen Therapie behandelt worden waren, traten bei 14 Patienten (93,33\%) keine neuen Metastasen auf, ein Patient $(6,67 \%)$ entwickelte neue Metastasen. Keiner dieser Patienten entwickelte ein Rezidiv.

In Abbildung 26 sind die Verhältnisse des Patientenkollektivs, das eine Monotherapie mit SAA erhalten hatte, graphisch dargestellt. 


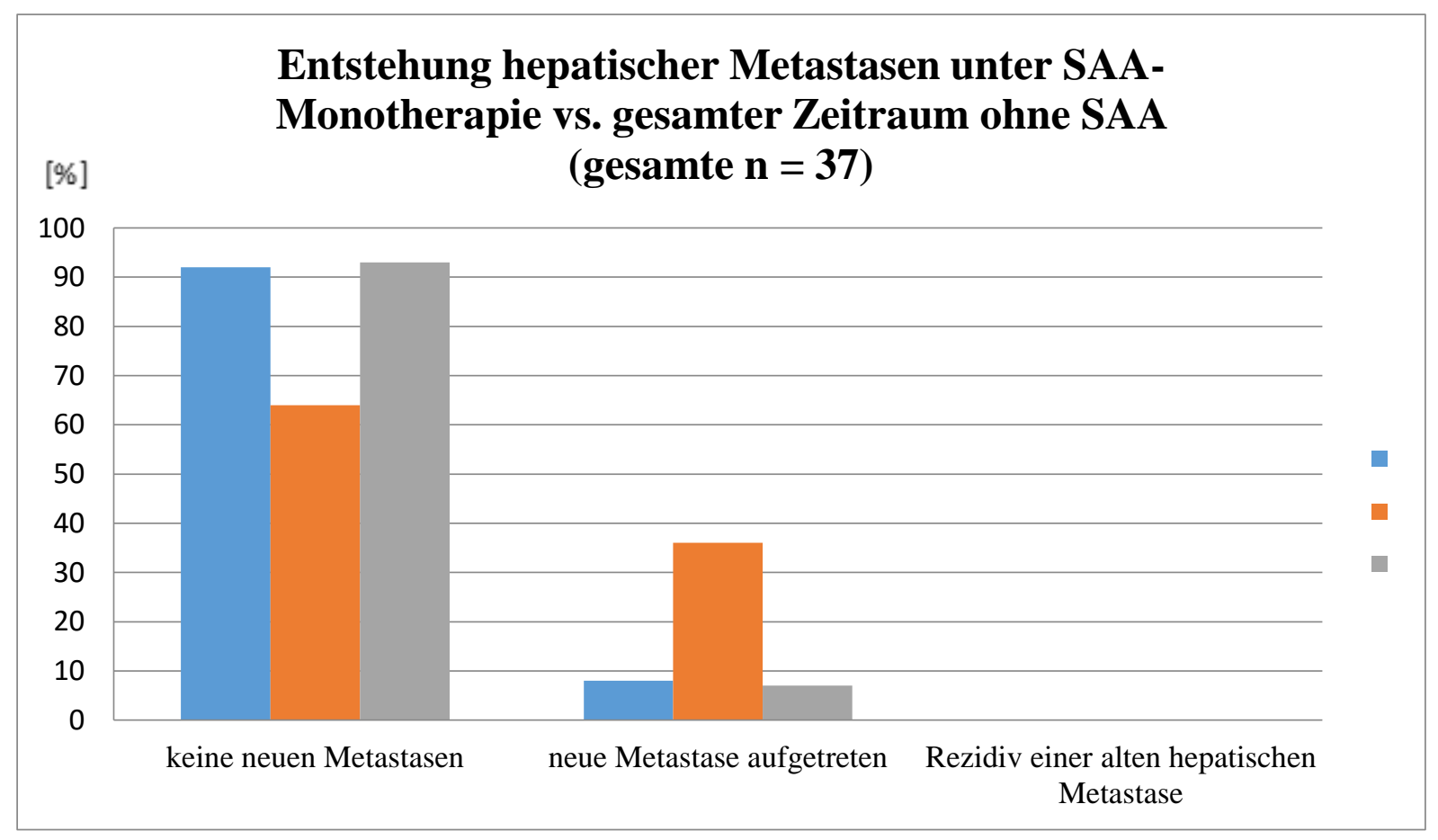

Abbildung 26: Entstehung hepatischer Metastasen im Kollektiv der 37 Patienten mit SAA-Monotherapie verglichen mit dem gesamten Zeitraum ohne SAA, unterteilt in die Gruppen "ohne jegliche Therapie" (22 Patienten) und "alternative Therapie" (15 Patienten)

\section{$\underline{\text { Mit Zusatztherapie }}$}

29 Patienten hatten während der Therapie mit SAA noch eine zusätzliche Therapie erhalten. Davon entwickelten 27 Patienten (93,1 \%) keine neuen Metastasen, bei zwei Patienten $(6,88 \%)$ traten neue Metastasen auf. Keiner dieser Patienten entwickelte ein Rezidiv.

Im Zeitraum ohne SAA blieben 18 Patienten der oben genannten 29 Patienten ohne jegliche Therapie, von denen 17 Patienten (94,44\%) keine neuen Metastasen entwickelten und bei einem Patienten $(5,55 \%)$ neue Metastasen auftraten. Ein Rezidiv konnte bei keinem der Patienten beobachtet werden. Unter den elf Patienten, die eine alternative Therapie erhalten hatten, blieben acht $(72,73 \%)$ ohne Metastasen, zwei $(18,18 \%)$ entwickelten neue Metastasen und ein Patient $(9,09 \%)$ entwickelte ein Rezidiv.

Abbildung 27 stellt diese Verhältnisse für das Kollektiv der Patienten mit SAA plus Zusatztherapie dar. 


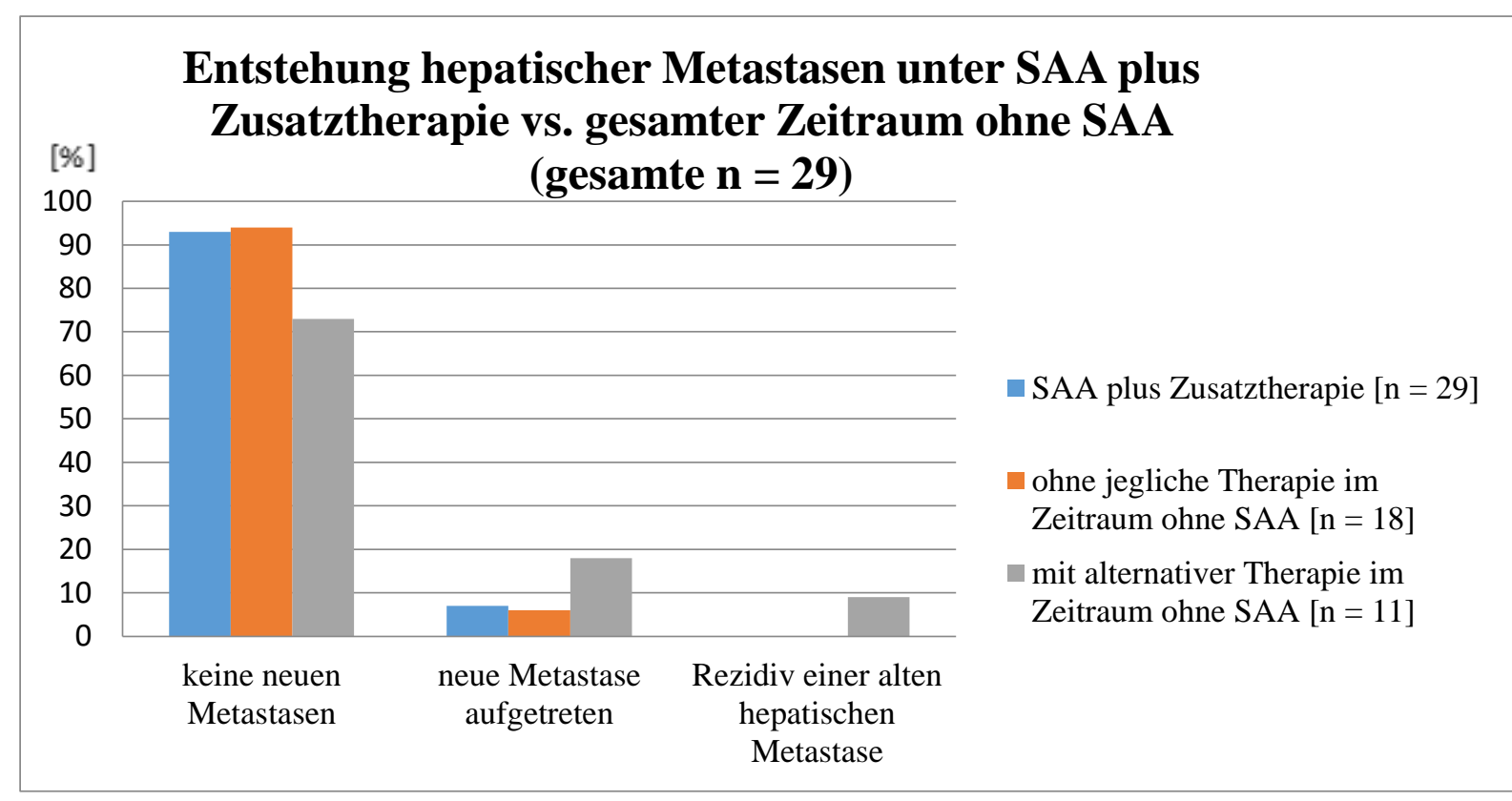

Abbildung 27: Entstehung hepatischer Metastasen im Kollektiv der 29 Patienten mit SAA plus Zusatztherapie verglichen mit dem Zeitraum ohne SAA, unterteilt in die Gruppen „ohne jegliche Therapie“ (18 Patienten) und "alternative Therapie" (elf Patienten)

\subsubsection{Größenverlauf der hepatischen Metastasen}

\section{Zeitraum ohne SAA}

Im Zeitraum ohne SAA konnte bei 18 Patienten eine stable disease der hepatischen Metastasen beobachtet werden $(27,27 \%)$. Hier enthalten sind auch diejenigen Patienten, die zum Zeitpunkt der Erstdiagnose noch keine hepatischen Metastasen gebildet hatten und weiterhin frei von solchen Metastasen geblieben sind. Bei einem Patienten trat eine partial response auf (1,52\%), eine progressive disease der hepatischen Metastasen wurde bei 44 Patienten beobachtet $(66,67 \%)$, Ein Rezidiv einer alten, bereits durch eine frühere Therapie verschwundenen Metastase wurde bei drei Patienten beobachtet (4,54\%).

\section{Zeitraum mit SAA}

Während der Therapie mit SAA konnte bei 22 Pateinten eine stable disease der hepatischen Metastasen beobachtet werden (33,33\%). Dies beinhaltet auch diejenigen Patienten, die zum Zeitpunkt der Erstdiagnose noch keine hepatischen Metastasen gebildet hatten und weiterhin frei von solchen Metastasen blieben. Drei Patienten erfuhren eine partial response $(4,54 \%)$, bei 41 Patienten wurde eine progressive disease der hepatischen Metastasen beobachtet $(62,12 \%)$. Ein Rezidiv einer früheren Metastase war unter der Therapie mit SAA bei keinem Patienten zu beobachten.

Diese Ergebnisse zeigt auch Abbildung 28. 


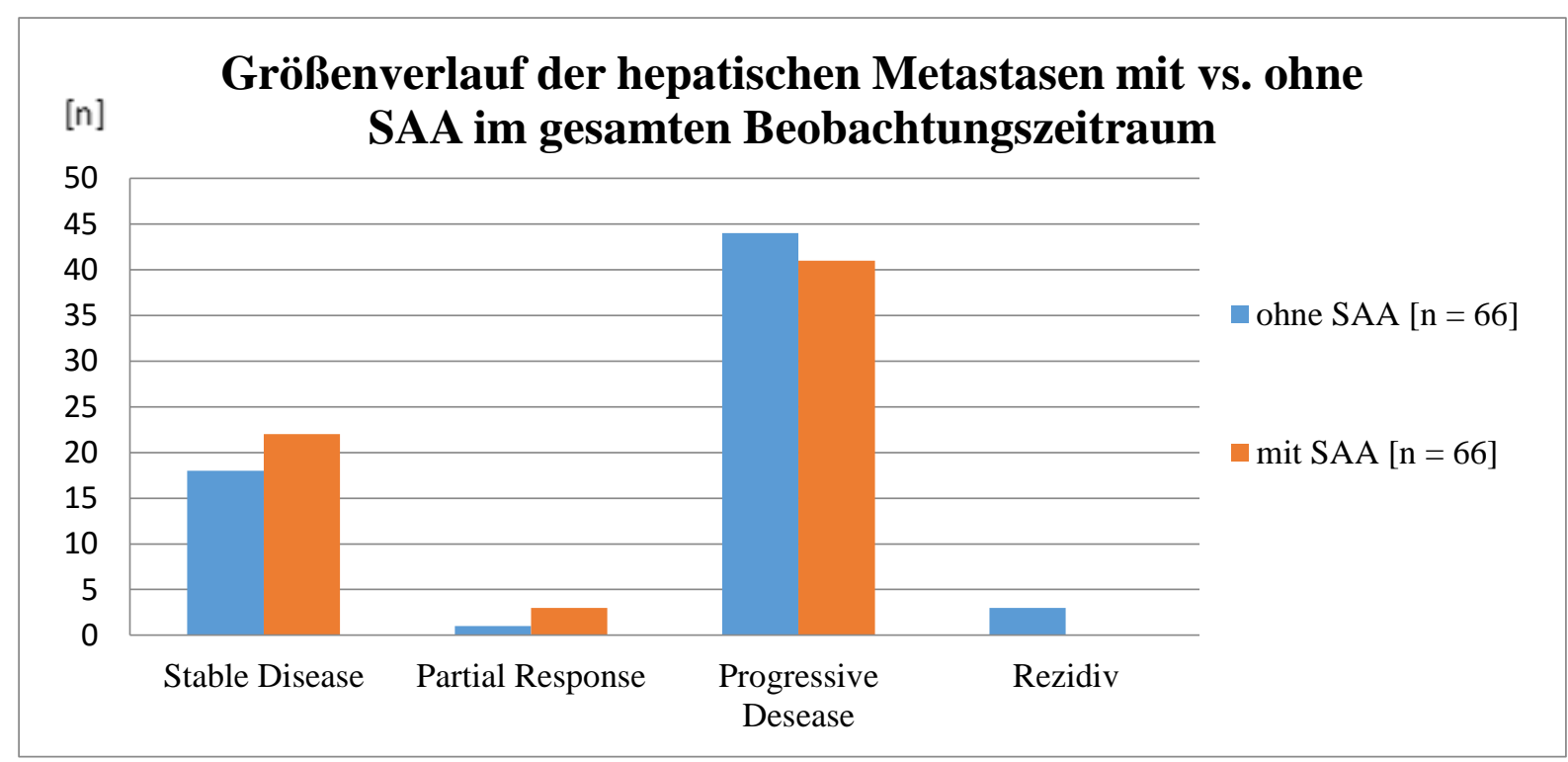

Abbildung 28: Größenverlauf der hepatischen Metastasen in den Zeiträumen mit bzw. ohne SAA im gesamten Beobachtungszeitraum im Vergleich $(n=66)$

Ohne Zusatztherapie

Für die 37 Patienten unter Monotherapie mit SAA ergibt sich folgender Größenverlauf der hepatischen Metastasen: 13 Patienten (35,15\%) zeigten eine stable disease, ein Patient $(2,7 \%)$ eine partial response, 23 Patienten $(62,16 \%)$ eine progressive disease. Bei keinem dieser Pateinten trat ein Rezidiv einer bereits behandelten hepatischen Metastase auf.

Was den Zeitraum ohne SAA für dieses Patientenkollektiv betrifft, so zeigte sich unter den 22 Patienten ohne jegliche Therapie eine stable disease bei sechs Patienten (27,27\%), eine partial response bei keinem der Patienten und eine progressive disease bei 14 Patienten $(63,64 \%)$. Ein Rezidiv trat bei zwei Patienten $(9,09 \%)$ auf. Von den 15 Patienten, die eine alternative Therapie erhalten hatten, erfuhren vier Patienten $(26,67 \%)$ eine stable disease, ein Patient $(6,67 \%)$ eine partial response und neun Patienten $(60 \%)$ eine progressive disease. Bei einem Patienten dieses Kollektivs (6,67\%) konnte das Auftreten eines Rezidivs beobachtet werden. Diese soeben beschriebenen Verhältnisse zeigt auch Abbildung 29. 


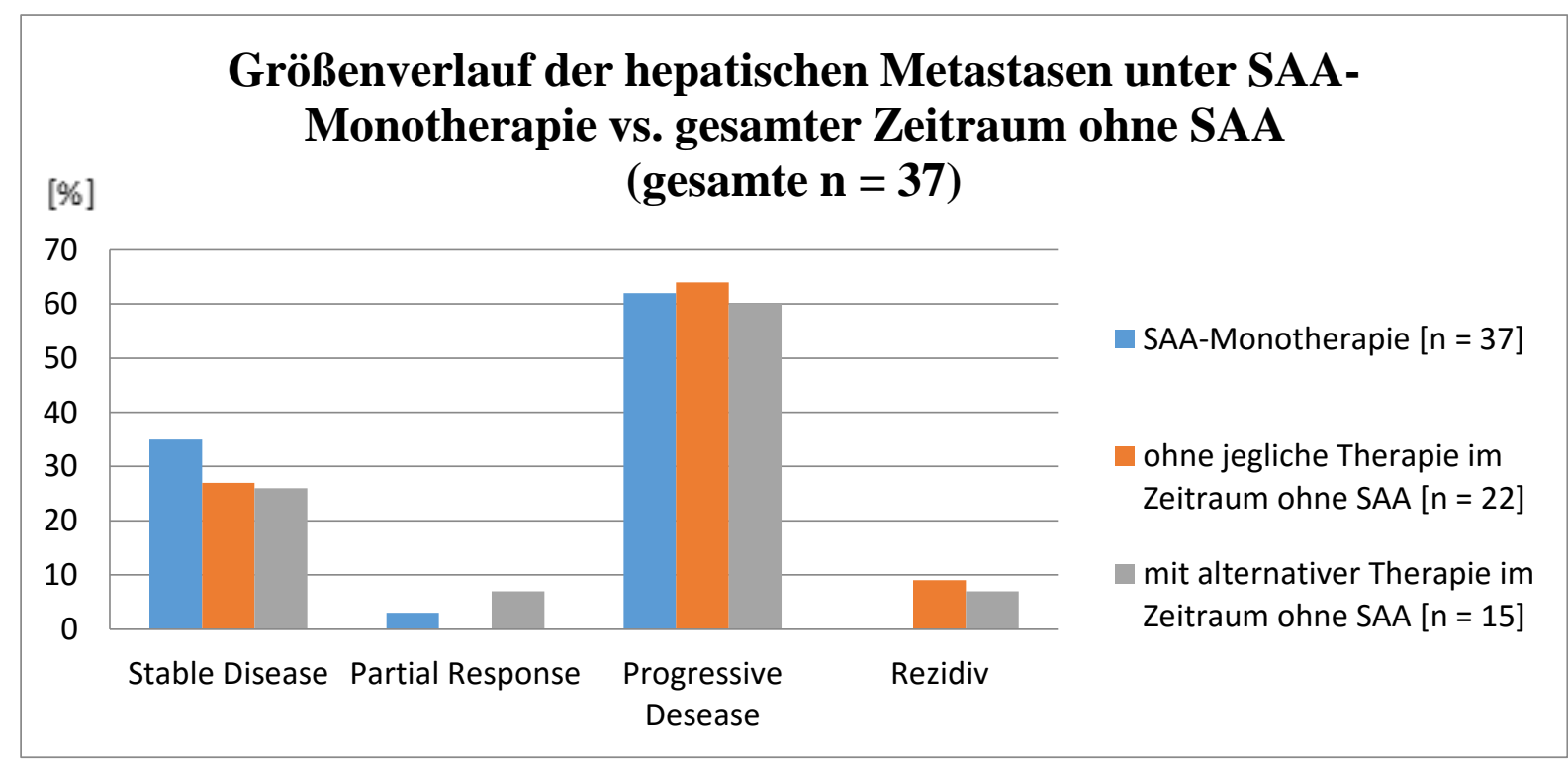

Abbildung 29: Größenverlauf der hepatischen Metastasen im Kollektiv der 37 Patienten mit SAA-Monotherapie verglichen mit dem gesamten Zeitraum ohne SAA, unterteilt in die Gruppen „ohne jegliche Therapie" (22 Patienten) und "alternative Therapie" (15 Patienten)

\section{$\underline{\text { Mit Zusatztherapie }}$}

Bei den 29 Patienten, die zu SAA noch eine Zusatztherapie erhalten hatten, zeigten sich neun Patienten $(31,03 \%)$ in der stable disease, zwei Patienten $(6,9 \%)$ in der partial response und 18 Patienten $(62,07 \%)$ in einer progressive disease. Keiner dieser Patienten entwickelte ein Rezidiv.

Im selben Kollektiv konnte für den Zeitraum ohne SAA unter den 18 Patienten ohne jegliche Therapie eine stable disease bei sechs Patienten $(33,33 \%)$, eine partial response bei keinem der Patienten und eine progressive disease bei zwölf Patienten $(66,67 \%)$ beobachtet werden. Bei keinem dieser Patienten trat ein Rezidiv auf. Bei den elf Patienten, die eine alternative Therapie erhalten hatten ergab sich eine stable disease bei zwei Patienten (18,18\%), eine partial response trat bei keinem der Patienten ein. Eine progressive disease entwickelten neun Patienten (81,82 \%). Bei keinem Patienten trat ein Rezidiv einer bereits behandelten hepatischen Metastase auf.

In Abbildung 30 sind die Verhältnisse des beschriebenen Patientenkollektivs unter SAA plus Zusatztherapie dargestellt. 


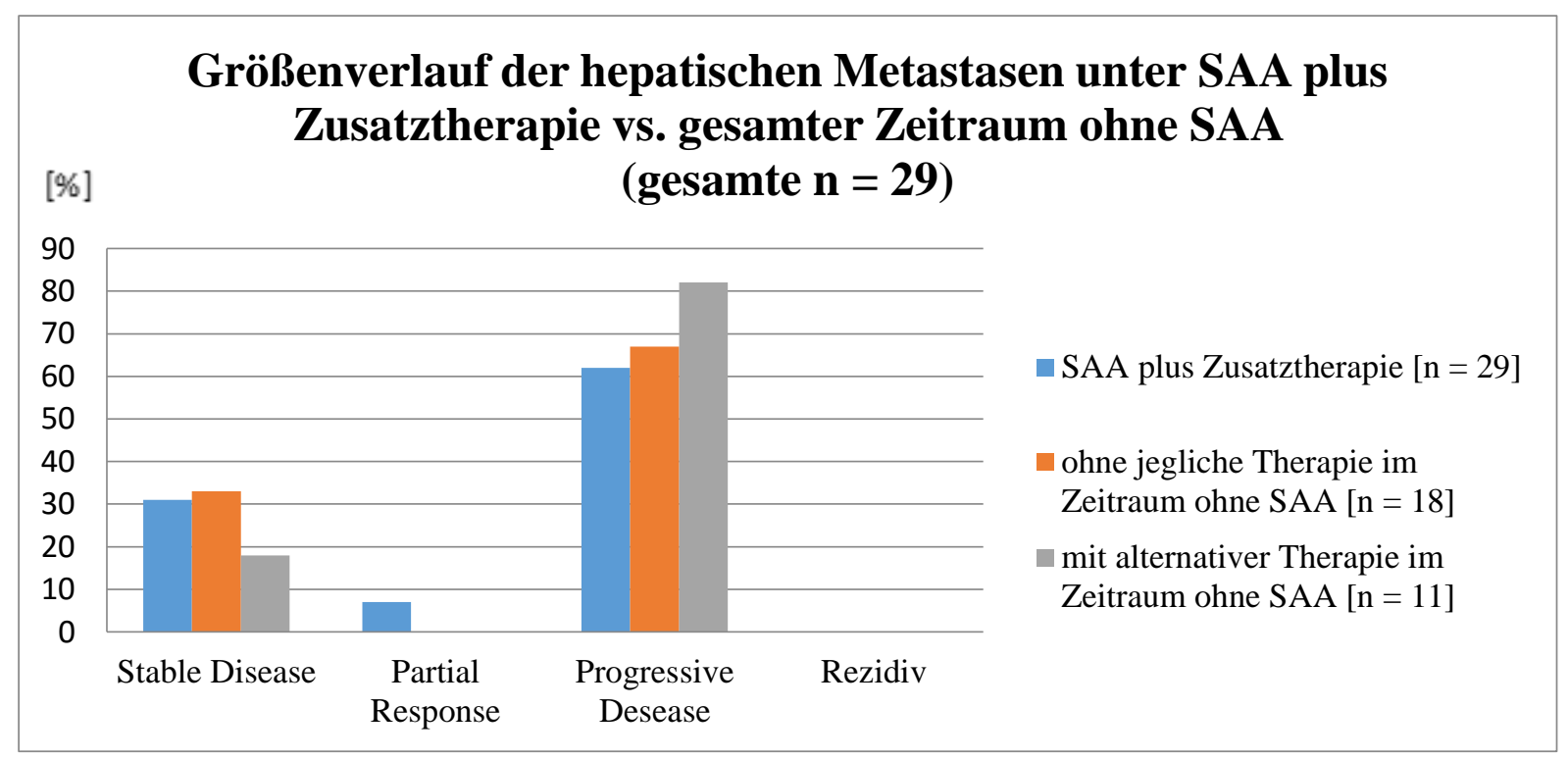

Abbildung 30: Größenverlauf der hepatischen Metastasen im Kollektiv der 29 Patienten mit SAA plus Zusatztherapie verglichen mit dem gesamten Zeitraum ohne SAA, unterteilt in die Gruppen „ohne jegliche Therapie“ (18 Patienten) und „alternative Therapie" (elf Patienten)

\subsubsection{Entstehung sonstiger peripherer Metastasen}

Zeitraum ohne SAA

Im Zeitraum ohne eine Therapie mit SAA entwickelten 27 Patienten neue periphere Metastasen $(40,9 \%)$. Bei 39 Patienten traten während dieses Zeitraums keine weiteren peripheren Metastasen auf $(59,1 \%)$.

\section{Zeitraum mit SAA}

Während der Therapie mit SAA entwickelten 16 Patienten neue periphere Metastasen $(24,24 \%)$. Bei 50 Patienten konnten während dieses Zeitraums keine neuen peripheren Metastasen nachgewiesen werden $(75,76 \%)$.

Abbildung 31 zeigt diese Verhältnisse.

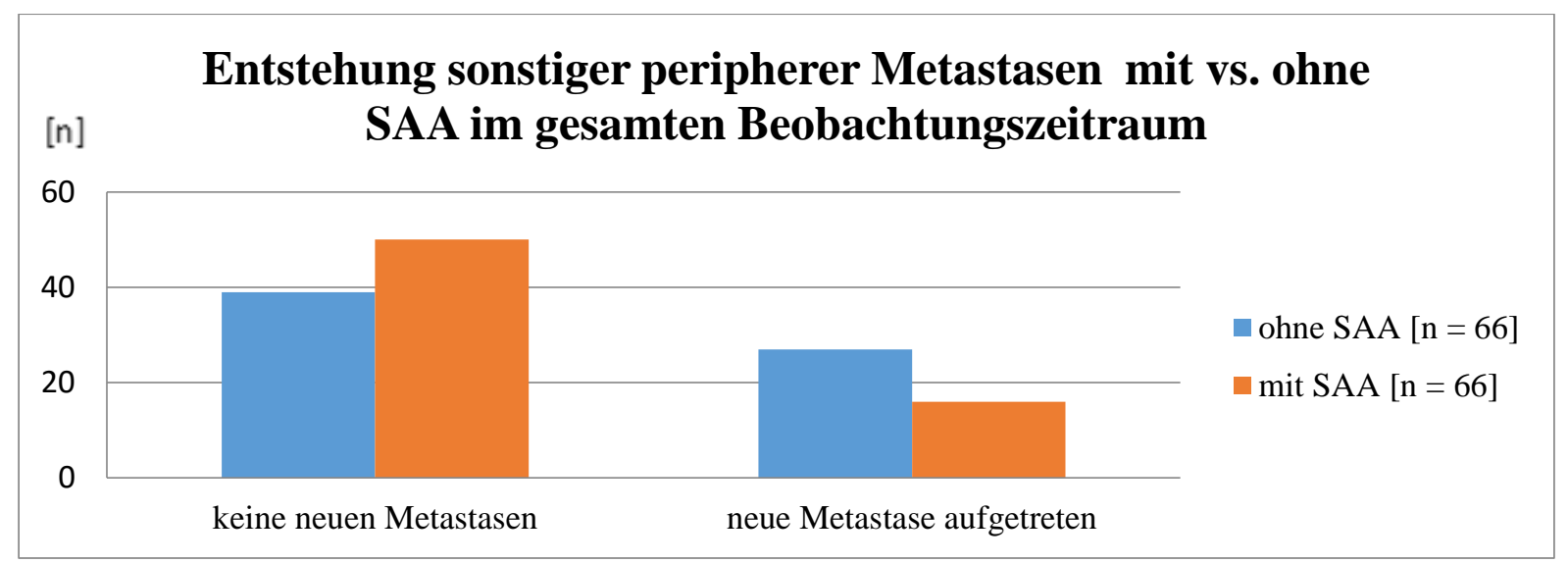

Abbildung 31: Entstehung sonstiger peripherer Metastasen im Zeitraum mit bzw. ohne SAA im gesamten Beobachtungszeitraum im Vergleich $(n=66)$ 
Ohne Zusatztherapie

Unter den 37 Patienten unter SAA-Monotherapie entwickelten 31 Patienten (83,78\%) keine neuen peripheren Metastasen wohingegen bei sechs Patienten (16,23\%) das Auftreten solcher Metastasen beobachtet werden konnte.

Unter den 22 Patienten, die im Zeitraum ohne SAA ohne jegliche Therapie blieben, kam es bei 14 Patienten $(63,64 \%)$ zu keinem Auftreten neuer peripherer Metastasen, acht Patienten (36,36 \%) entwickelten jedoch solche Metastasen. In der Gruppe der 15 Patienten, die im Zeitraum ohne SAA eine alternative Therapie erhalten hatten, konnte ein Wegbleiben neuer peripherer Metastasen bei sieben Patienten (46,67 \%) und ein Auftreten solcher Metastasen bei acht Patienten $(53,34 \%)$ beobachtet werden.

Abbildung 32 zeigt diese Verhältnisse.

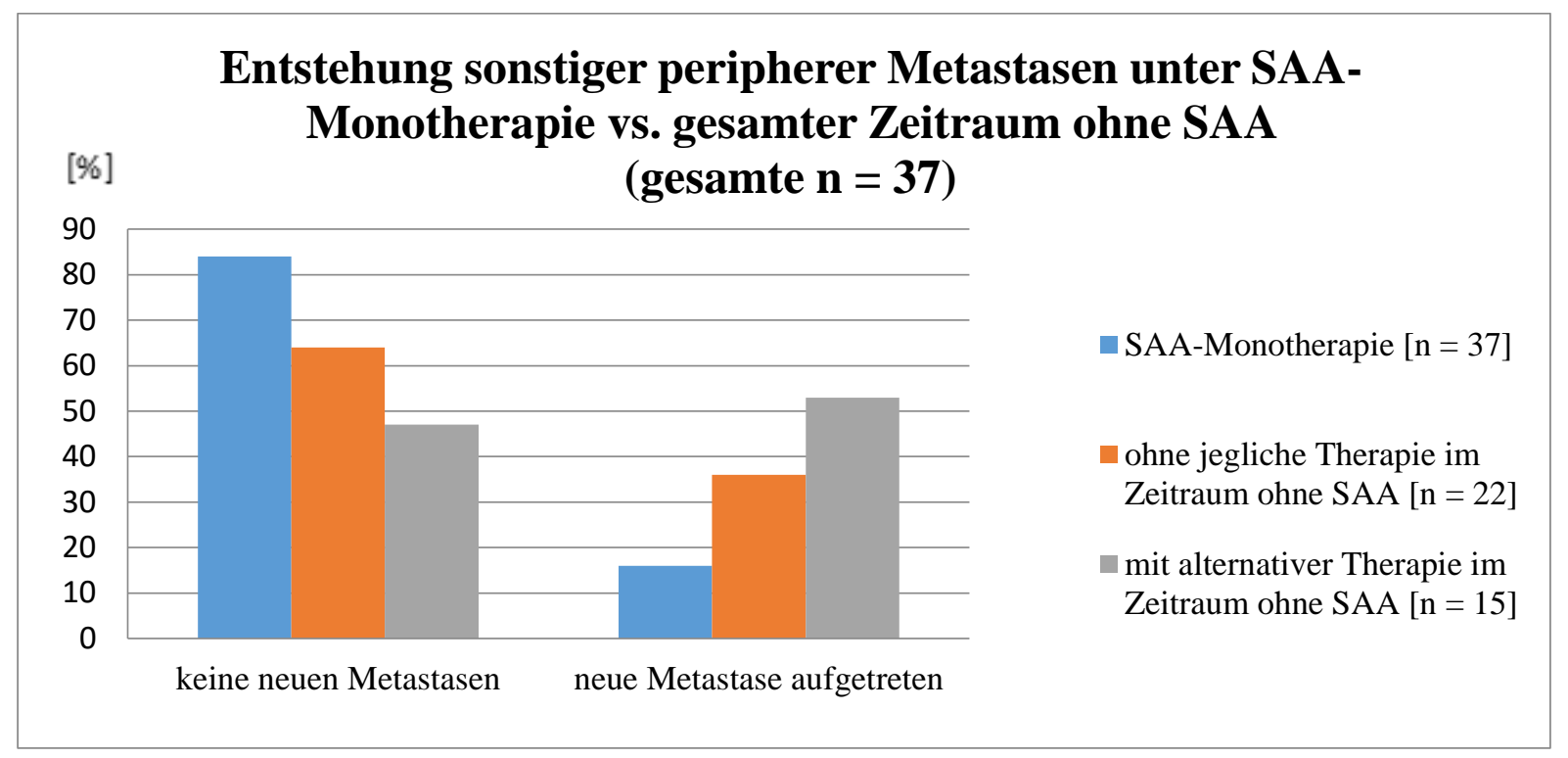

Abbildung 32: Entstehung sonstiger peripherer Metastasen im Kollektiv der 37 Patienten mit SAA-Monotherapie verglichen mit dem gesamten Zeitraum ohne SAA, unterteilt in die Gruppen "ohne jegliche Therapie" (22 Patienten) und "alternative Therapie" (15 Patienten)

\section{$\underline{\text { Mit Zusatztherapie }}$}

In der Gruppe der 29 Patienten, die SAA plus Zusatztherapie erhalten hatten, zeigten sich keine neuen peripheren Metastasen bei 19 Patienten (65,52 \%). Zehn Patienten (34,48 \%) entwickelten solche Metastasen.

Für den Zeitraum ohne SAA ergab sich folgendes Verhältnis: Unter den 18 Patienten, die ohne jegliche Therapie blieben, traten bei zehn Patienten (56,56 \%) keine neunen Metastasen auf, wohingegen bei acht Patienten (44,44\%) ein solches Auftreten beobachtet werden konnte. Acht Patienten $(72,73 \%)$ der elf Patienten, die eine alternative Therapie erhalten hatten, 
blieben ohne neue periphere Metastasen. Bei drei Patienten (27,27 \%) kam es zur Entwicklung solcher Metastasen.

In Abbildung 33 werden die soeben genannten Verhältnisse bezogen auf die Gruppe mit SAA plus Zusatztherapie graphisch dargestellt.

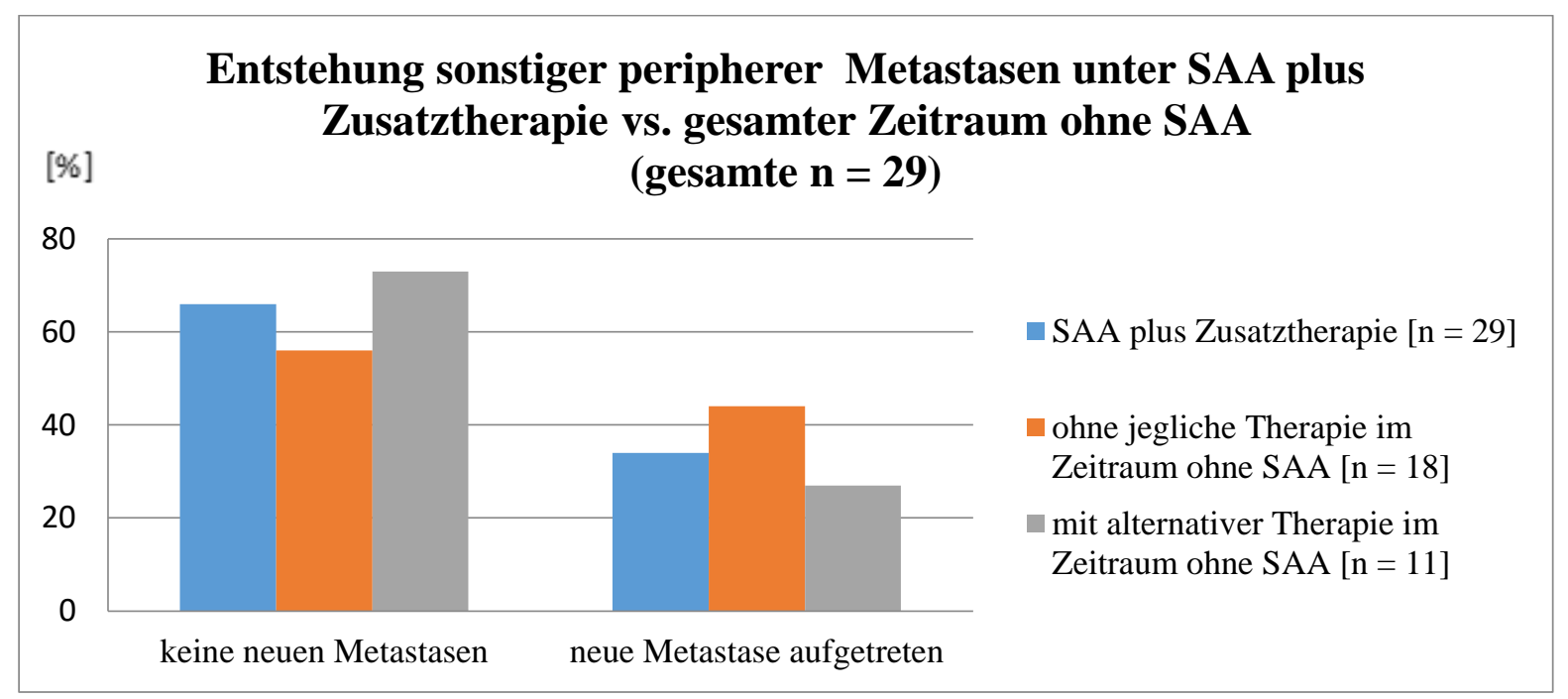

Abbildung 33: Entstehung sonstiger peripherer Metastasen im Kollektiv der 29 Patienten mit SAA plus Zusatztherapie verglichen mit dem gesamten Zeitraum ohne SAA, unterteilt in die Gruppen „ohne jegliche Therapie" (18 Patienten) und "alternative Therapie" (elf Patienten)

\subsubsection{Größenverlauf sonstiger peripherer Metastasen}

\section{Zeitraum ohne SAA}

Im Zeitraum ohne Therapie mit SAA konnte bei 53 Patienten eine stable disease der vorhandenen peripheren Metastasen beobachtet werden (80,3\%). Dies beinhaltet auch, dass Patienten, die zum Zeitpunkt der Erstdiagose noch keine Metastasen hatten, auch im Verlauf dieses Zeitraums keine Metastasen entwickelten. Bei 13 Patienten war eine progressive disease zu beobachten $(19,7 \%)$. Partial response oder Rezidive traten bei keinem Patienten auf.

\section{$\underline{\text { Zeitraum mit SAA }}$}

Während der Therapie mit SAA konnte bei 56 Patienten eine stable disease der peripheren Metastasen dokumentiert werden (84,85\%). Dies beinhaltet auch, dass Patienten, die zum Zeitpunkt der Erstdiagnose noch keine Metastasen hatten, auch im Verlauf dieses Zeitraums keine Metastasen entwickelten. Bei neun Patienten konnte eine progressive disease beobachtet werden $(13,64 \%)$. Partial response oder Rezidive traten bei keinem Patienten auf.

Abbildung 34 stellt den oben genannten Vergleich dar. 


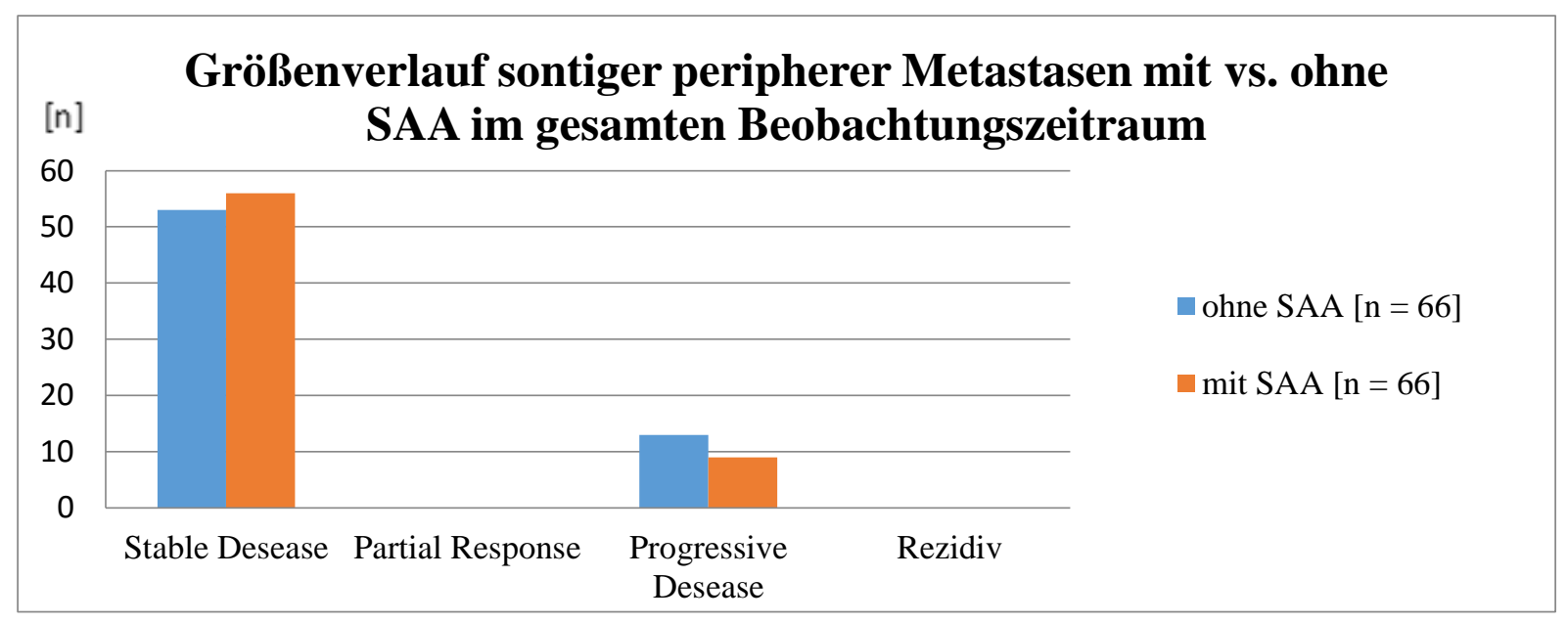

Abbildung 34: Größenverlauf sonstiger peripherer Metastasen mit bzw. ohne SAA im gesamten Beobachtungszeitraum im Vergleich $(n=66)$

\section{Ohne Zusatztherapie}

Für die Gruppe der 37 Patienten unter Monotherapie mit SAA und den Größenverlauf der sonstigen peripheren Metastasen ergab sich folgende Verteilung: 34 Patienten (91,89 \%) zeigten eine stable disease, kein Patient zeigte eine partial response. Zwei Patienten $(5,41 \%)$ erfuhren eine progressive disease und bei keinem der Patienten kam es zu einem Rezidiv einer bereits behandelten peripheren Metastase. Von einem Patienten $(2,7 \%)$ fehlen die Daten bezüglich dieser Auswertung.

Im selben Kollektiv und für den Zeitraum ohne SAA zeigten 19 Patienten (86,36 \%) der 22 Patienten ohne jegliche Therapie eine stable disease und keiner dieser Patienten eine partial response. Bei drei Patienten (13,64 \%) konnte eine progressive disease beobachtet werden, keiner der Patienten entwickelte ein Rezidiv. Unter den 15 Patienten, die eine alternative Therapie erhalten hatten, blieben 13 Patienten (86,67\%) bezüglich der sonstigen peripheren Metastasen in der stable disease. Keiner der Patienten zeigte eine partial response. Bei zwei Patienten $(13,33 \%)$ kam es zur progressive disease. Bei keinem dieser Patienten trat ein Rezidiv auf.

Oben genannte Verhältnisse sind in Abbildung 35 verdeutlicht. 


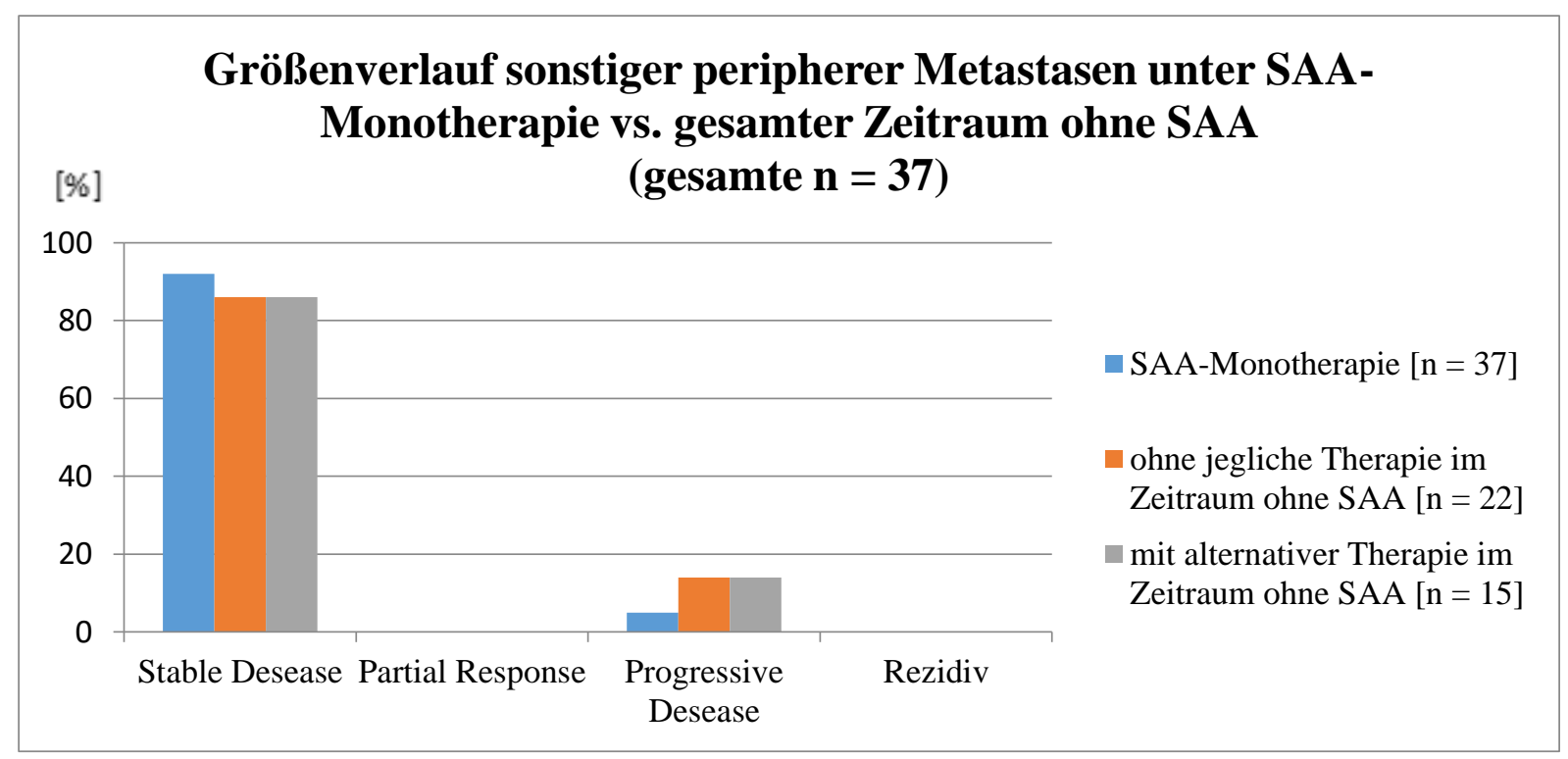

Abbildung 35: Größenverlauf sonstiger peripherer Metastasen im Kollektiv der 37 Patienten mit SAA-Monotherapie verglichen mit dem gesamten Zeitraum ohne SAA, unterteilt in die Gruppen „ohne jegliche Therapie“ (22 Patienten) und „alternative Therapie" (15 Patienten)

\section{$\underline{\text { Mit Zusatztherapie }}$}

Bezüglich der 29 Patienten, die SAA plus Zusatztherapie erhalten hatten, und den Größenverlauf der sonstigen peripheren Metastasen konnten folgende Beobachtungen gemacht werden: Bei 22 Patienten $(75,86 \%)$ zeigte sich eine stable disease, bei keinem der Patienten eine partial response. Sieben Patienten (24,14\%) erfuhren eine progressive disease, keiner dieser Patienten entwickelte ein Rezidiv.

Im selben Kollektiv und für den Zeitraum ohne SAA kam es bei elf Patienten $(61,11 \%)$ der 18 Patienten ohne jegliche Therapie zu einer stable disease, bei keinem dieser Patienten zu einer partial response, bei sieben Patienten $(38,89 \%) \mathrm{zu}$ einer progressive disease und bei keinem der Patienten zur Entwicklung eines Rezidivs. In der Gruppe der elf Patienten, bei denen eine alternative Therapie durchgeführt worden war, war bei zehn Patienten $(90,91 \%)$ eine stable disease, bei keinem dieser Patienten eine partial response, bei einem Patienten $(9,09 \%)$ eine progressive disease und bei keinem Patienten ein Rezidiv zu beobachten.

Die hier aufgeführten Verhältnisse werden in Abbildung 36 graphisch aufgearbeitet. 


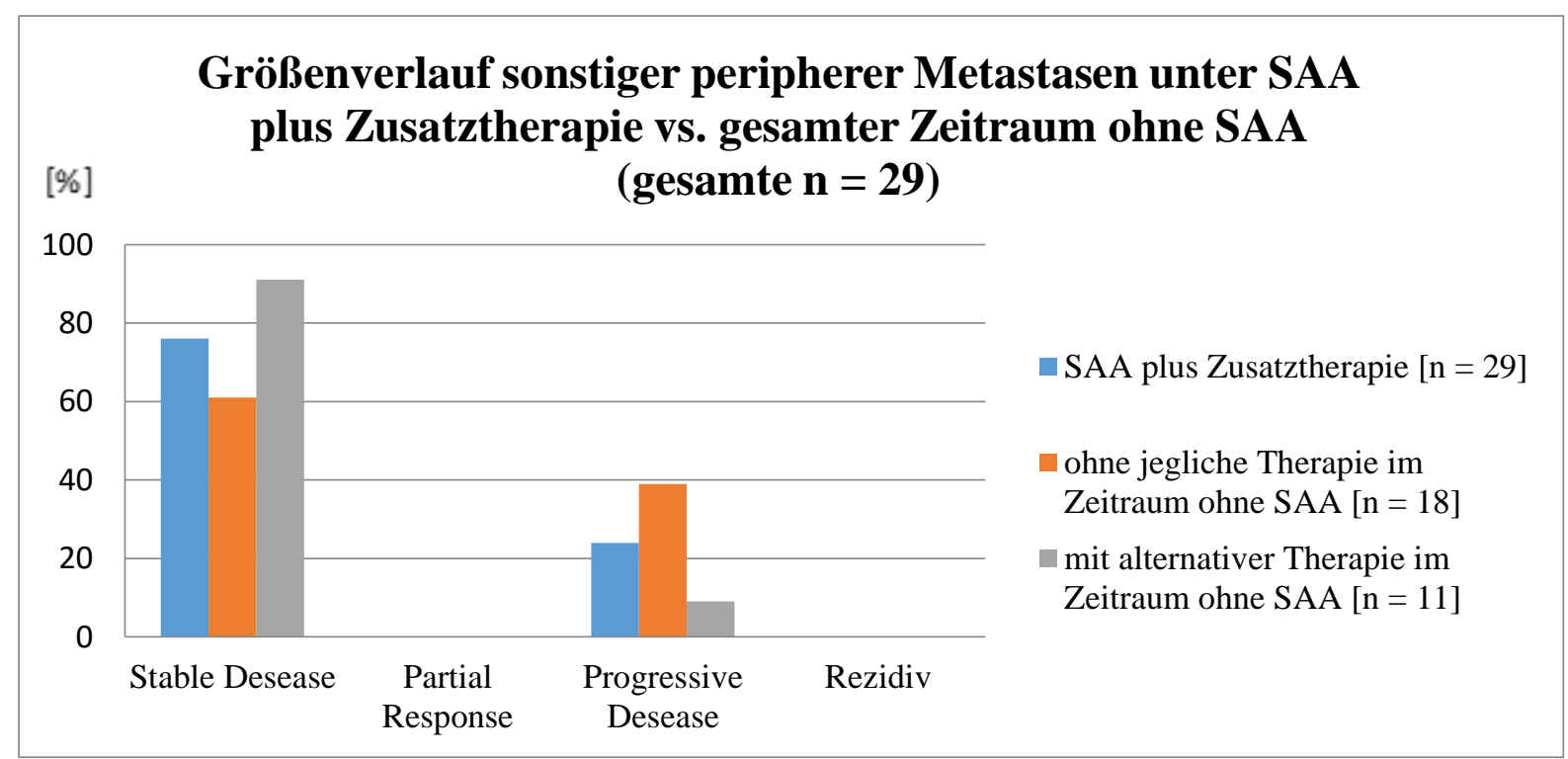

Abbildung 36: Größenverlauf sonstiger peripherer Metastasen im Kollektiv der 29 Patienten mit SAA plus Zusatztherapie verglichen mit dem gesamten Zeitraum ohne SAA, unterteilt in die Gruppen „ohne jegliche Therapie“ (18 Patienten) und "alternative Therapie" (elf Patienten) 


\section{Diskussion}

In der Behandlung von NET spielen verschiedene Therapiestrategien eine Rolle: Neben SAA zur Kontrolle der durch den Tumor hervorgerufenen Symptomatik, werden vor allem Therapiestrategien mit dem Ziel einer Verminderung der Tumorlast eingesetzt. Zytoreduktive, antiproliferative Therapiestrategien beinhalten systemische Chemotherapie, chirurgische Resektion und Debulking, Embolisation und Resektion von Lebermetastasen und in weit fortgeschrittenen Stadien der hepatischen Metastasierung in ausgewählten Fällen auch die Lebertransplantation. Jedoch birgt vor allem die Chemotherapie durch ihre hohe Toxizität und das damit verbundene Potential schwerwiegender Nebenwirkungen viele Probleme. Dies ist Anlass zur Suche nach Therapiealternativen.

Mehrere Studien schreiben der Therapie mit SAA neben der Symptomkontrolle auch einen Effekt auf das Tumorwachstum zu (Arnold et al. 1996; Ducreux et al. 2000; Rinke et al. 2009). Gleichzeitig konnte in mehreren Studien nachgewiesen werden, dass zwischen Symptomkontrolle, Verlauf der Tumormarker und Tumorwachstum keine Korrelation besteht (Arnold et al. 1996; Garland et al. 2003; Wang et al. 2006), was auch an den Daten unserer Analyse sichtbar wird. Dies mag an den unterschiedlichen Effekten, die Somatostatin auf Zelle und Körper ausübt, liegen. So stehen die Rezeptoren sst $_{2}$ und sst $\mathrm{im}_{5}$ Vordergrund, wenn es um die Kontrolle der Hormonsekretion und damit um die Symptomkontrolle geht. $\mathrm{Sst}_{2}$ und sst $_{3}$ spielen eine wichtige Rolle in der Kontrolle des Tumorwachstums: Sst ${ }_{2}$ vermittelt unter anderem die Expression von p27 (Pagès et al. 1999), was im Zellzyklus den Übergang von der $\mathrm{G}_{0}$-Phase in die $\mathrm{G}_{1}$-Phase hemmt und somit einen zytostatischen Effekt hervorruft. Für sst 3 konnte von Sharma und Srikant (1998) sowohl ein zytotoxischer, als auch ein zytostatischer Effekt in Mammakarzinom-Zellen nachgewiesen werden. Vermutet wird eine durch $\mathrm{sst}_{3}$ hervorgerufene Expression von p53 und Bax, was zum Zellzyklusarrest in der G0-Phase beziehungsweise zur Induktion der Apoptose führt. Eriksson et al. (1997) untersuchten in ihrer Studie Biopsien aus Lebermetastasen neuroendokrinen Ursprungs vor und während einer Therapie mit SAA, wobei mit zunehmender Therapiedauer ein Anstieg der Anzahl apoptotischer Zellen zu beobachten war.

Entscheidend für die Kontrolle des Tumorwachstums ist außerdem eine Hemmung der Serotoninfreisetzung im Hypothalamus, was zu einer Hemmung der Freisetzung von Wachstumsfaktoren in der Hypophyse führt. Eine besondere Rolle dabei spielt IGF-1. Verminderte Konzentrationen an IGF-1 können auch durch SAA erreicht werden. Dies mag den vermuteten Effekt der Wachstumskontrolle unter SAA erklären. Der Einsatz von SAA zur Hemmung der Freisetzung von Wachstumsfaktoren und damit zur Reduktion der IGF-1-Konzentration ist 
auch in der Therapie der Akromegalie weit verbreitet (vgl. Stella, Therapieleitfaden Hypophysenerkrankungen, 2006).

Im Rahmen der vorliegenden Untersuchung wurde der Krankheitsverlauf von 66 Patienten, die an einem NET erkrankt waren und in der Universitätsmedizin Göttingen zwischen 1. Januar 1975 und 31. August 2011 mit SAA behandelt wurden, beobachtet und analysiert. Dabei wurden vor allem der Verlauf der Tumorgröße und die Dauer der stable disease in den Zeiträumen mit vs. ohne Therapie von SAA miteinander verglichen und statistisch ausgewertet. Vergleiche der beiden genannten Zeiträume fanden zur Beurteilung des gesamten Verlaufs der Tumorgröße und dessen Ausbreitung aber auch bezüglich des Verlaufs von Symptomatik, Tumormarkern und Metastasierung statt.

Unsere Ergebnisse sollen nun in den Kontext aktueller Literatur gestellt und anhand dieser diskutiert werden.

\subsection{Demographie}

In unseren Beobachtungen dominieren die männlichen Patienten mit 63,64 \% (n=42) gegenüber den weiblichen Patienten mit 36,36 \% $(n=24)$. In den Beobachtungen der Literatur liegt meist ein ausgeglichenes Verhältnis von männlichen und weiblichen Patienten vor, wobei auch hier eine leichte Dominanz der männlichen Patienten zu beobachten ist: Das Patientenkollektiv von Arnold et al. (1996) beispielsweise zeigt einen Anteil männlicher Patienten von $52,4 \%(\mathrm{n}=54)$, wohingegen 47,6\% $(\mathrm{n}=49)$ weiblich waren. Durch die geringe Patientenanzahl unseres Kollektivs lässt sich die doch deutlichere Dominanz männlicher Patienten begründen: Schaut man sich hierzu die Studie von Ducreux et al. (2000) an, so zeigt sich bei einem Kollektiv von 46 Patienten ein Verhältnis von 65,2 \% $(n=30)$ männlicher zu 34,8 \% (n = 16) weiblicher Patienten.

Das in der Literatur beschriebene Durchschnittsalter bei Erstdiagnose (zwischen 55 und 58 Jahren) stimmt mit dem durchschnittlichen Alter unserer Beobachtungen von 55 Jahren überein. In der Altersverteilung ergaben sich ein Minimum von 25 Jahren und ein Maximum von 77 Jahren. Dies spiegelt die beiden bekannten Altersgipfel für das Auftreten von NET, wonach das Alter bei Erstdiagnose bevorzugt zwischen dem 15. und 25. Lebensjahr und zwischen dem 65. und 75. Lebensjahr liegt, wider (Bajetta et al. 2009; siehe auch Kapitel 1.1.1). Betrachtet man den Zeitraum zwischen Erstdiagnose und Beginn einer Therapie mit SAA, so wurden Patienten in unserem Kollektiv zu einem vergleichsweise frühen Zeitpunkt therapiert: Unsere Beobachtung ergab für diesen Zeitraum eine mittlere Dauer von 3,53 Monaten. In der Studie von Rinke et al. (2009) wurden Patienten erst nach durchschnittlich 7,5 Monaten nach 
Diagnosestellung therapiert. Geht man davon aus, dass SAA vor allem zur Symptomkontrolle eingesetzt werden, so mag dieser deutlich kürzere Zeitraum in unserer Beobachtung in der deutlich höheren Anzahl an Patienten mit funktionellen Tumoren begründet sein: Während in unserem Patientenkollektiv $68 \%$ der Patienten unter einem funktionellen Tumor litten, waren es im Kollektiv von Rinke et al. (2009) nur 39 \% (vergleiche auch Kapitel 4.1.4). Der Anteil an Patienten mit therapiebedürftiger Symptomatik, die einen raschen Therapiebeginn fordert, ist also in unserer Beobachtung höher, was zu einer Senkung der Durchschnittsdauer des genannten Zeitintervalls führt.

\subsubsection{Tumorlokalisation}

In unserer Analyse konnte eine Dominanz der Primärtumoren in Dünndarm und Pankreas beobachten werden (Dünndarm 37,88 \%, Pankreas 24,24 \%). Dies spiegelt sich auch in der Literatur wider: Das Patientenkollektiv von Ducreux et al. (2000) beschreibt einen Anteil von $50 \%$ an Primärtumoren im Dünndarm und $16 \%$ im Pankreas. Arnold et al. (1996) beschrieben in einer Studie eine Dominanz von $71 \%$ an im Dünndarm lokalisierten Primärtumoren.

\subsubsection{Grading bei Erstdiagnose}

In unserer Analyse konnten überwiegend Patienten mit einem gut bis mäßig differenzierten Tumor beobachtet werden: Von den 33 Patienten, bei denen das Grading im Rahmen der Analyse bekannt war, wiesen 27 Patienten einen G1- oder G2-Tumor auf (81,12\%). Dies spiegelt den Anwendungsbereich der SAA vor allem bei diesen Tumoren, die eine niedrigere Proliferationsrate und dadurch ein langsameres Wachstum aufweisen, wider. Wie in Kapitel 1.3.5 bereits erläutert, finden bei schlecht-differenzierten, schnell wachsenden Tumoren vermehrt Chemotherapeutika ihre Anwendung. Auch dies kann im Patientenkollektiv unserer Analyse beobachtet werden: Lediglich sechs der Patienten, bei denen das Grading bekannt war, wiesen einen G3-Tumor auf $(18,18 \%)$.

Es sei noch darauf hingewiesen, dass nur von $50 \%$ der Patienten des Göttinger Kollektivs überhaupt ein Grading bekannt ist. Bei den anderen $50 \%$ handelt es sich zumeist um Patienten, deren Diagnose schon Jahrzehnte zurückliegt. Die Bestimmung des Ki-67-Index, auf dem das Grading basiert, wurde erst 1992 eingeführt (siehe auch Kapitel 2.1.1). Bei Patienten, die vor diesem Zeitpunkt diagnostiziert wurden, entsprach die Bestimmung dieses Index noch nicht dem Standard, wurde folglich nicht routinemäßig durchgeführt und in den Krankenakten nicht dokumentiert. Aussagen über das bei ihnen bestehende Grading sind im Nachhinein leider nicht zu treffen. 


\subsubsection{Staging bei Erstdiagnose}

Im Patientenkollektiv unserer Analyse überwiegt der Anteil der Tumoren im T4-Stadium ebenso wie der Anteil der Patienten, die bei Erstdiagnose bereits Metastasen aufwiesen. Unter den bereits metastasierten Tumoren überwiegen die hepatisch metastasierten bei Weitem. Ein Überwiegen des T4-Stadiums und der bereits hepatisch metastasierten Tumoren lässt sich auch in der Literatur finden: Yao et al. beschrieben (2008) eine Quote von $50 \%$ bei Erstdiagnose bereits metastasierter Patienten. Eine Quote von $66 \%$ wurde in einer Studien von Strosberg et al. (2011 b) beschrieben, was den $68 \%$ bei Erstdiagnose metastasierter Patienten unsrer Analyse wohl am nächsten kommt.

Wie schon in Kapitel 1.2.1 erwähnt, ist dieser hohe Anteil an sowohl lokal als auch systemisch fortgeschrittenen Tumoren bei Erstdiagnose in der fehlenden Frühsymptomatik begründet.

\subsubsection{Funktionelle / nicht-funktionelle Tumoren}

Bei den von uns beobachteten Patienten findet sich eine Dominanz der funktionellen Tumoren von $68 \%$ gegenüber $32 \%$ nicht-funktioneller Tumoren. Dieses Verhältnis von 2:1 kann in der Literatur wiedergefunden, jedoch durch sie nicht eindeutig als charakteristisch beschrieben werden: In der Studie von Ducreux et al. (2000) herrscht ebenfalls ein Verhältnis von 2:1 funktioneller zu nicht-funktioneller Tumoren vor (64\% vs. $39 \%$ ). Das Patientenkollektiv von Rinke et al. (2009) hingegen weist eher ein Verhältnis von 1:2 auf (38,8\% funktionelle, 61,2 $\%$ nicht-funktionelle Tumoren). Dennoch sind unsere Ergebnisse übereinstimmend mit einer Beschreibung von Öberg und Castellano (2011), wonach 2/3 der GI-NET zur Gruppe der funktionellen NET gehören.

Bei der gezeigten Symptomatik der Patienten, die unter einem funktionellen Tumor litten, lässt sich ein Überwiegen der Flush-Symptomatik feststellen. Auch dies lässt sich in der Literatur wiederfinden: Dort wird die Flush-Symptomatik unter den Häufigkeiten der auftretenden Symptomatik an erster Stelle genannt. Je nach Autor werden Anteile zwischen $63 \%$ und $94 \%$ der an Flush leidenden Patienten unter den Patienten mit einem funktionellen NET beschrieben (Rubin et al. 1999; Schnirer et al. 2003; Kaltsas et al. 2004).

\subsubsection{Sonstige Therapie}

Die bei uns beobachtete hohe Rate der primär operativ behandelten Patienten von $63,6 \%$ ist auch in der Studie von Ducreux et al. (2000) wiederzufinden: 67,4 \% der Patienten wurden dort vor einer Therapie mit SAA operativ behandelt. Auch in der durch Rinke et al. (2009) durchgeführten PROMID-Studie wurde der Primärtumor bei $66 \%$ des Patientenkollektivs vor 
einer medikamentösen Behandlung operativ entfernt. Dies macht noch einmal deutlich, wie wichtig die primär chirurgische zytoreduktive Therapie ist (vergleiche auch Kapitel 1.3.1).

Betrachtet man die medikamentöse Therapie vor einem Beginn der Therapie mit SAA, so decken sich die Angaben des Patientenkollektivs dieser Analyse mit den Angaben des Patientenkollektivs der durch Arnold et al. (1996) durchgeführten Studie. Aus den beiden Kollektiven unserer Beobachtung und der Studie von Arnold et al. (1996) wird auch ersichtlich, dass in der primären Therapie neben SAA vor allem die Chemotherapie zum Einsatz kommt: Wird der Einsatz von Chemotherapeutika mit dem Einsatz von Interferon- $\alpha$ verglichen, so erhielten in unserer Analyse 13 Patienten eine Chemotherapie, wohingegen sieben Patienten mit Interferon- $\alpha$ therapiert wurden (hierunter befinden sich vier Patienten, die sowohl Chemotherapie als auch Interferon- $\alpha$ bekommen haben). In der Studie von Arnold et al. (1996) erhielten 21 Patienten eine Chemotherapie, neun Patienten wurden mit Interferon- $\alpha$ therapiert. Vor allem Karzinome höheren Gradings werden palliativ durch eine Chemotherapie behandelt, da sie durch ihr schnelles Wachstum dieser Therapie am zugänglichsten sind (vergleiche auch Kapitel 1.3.5). Der hohe Anteil an Patienten, die in unserem Kollektiv eine Chemotherapie erhielten, korreliert somit auch mit dem höheren Anteil an G2-/G3-Tumoren/Karzinomen gegenüber den G1-Tumoren unserer Beobachtung: Von den in die Beobachtung eingeschlossenen 66 Patienten konnte bei Erstdiagnose bei 23 Patienten ein Tumor im G2-/G3-Stadium nachgewiesen werden (34,8 \%), wohingegen nur bei 10 Patienten ein G1-Stadium histologisch gesichert werden konnte (15,2\%). Für die übrigen 33 Patienten liegen keine Daten der histologischen Untersuchung vor $(50 \%)$. (Detaillierte Aufschlüsselung der oben genannten Zahlen siehe Kapitel 3.1.2.)

Die bei den Patienten unserer Analyse angewandte Erstlinientherapie entspricht auch den aktuellen ENETS Consensus Guidelines von Pavel et al. (2012).

\subsection{Beobachtungsdauer}

Die mittlere Beobachtungsdauer beträgt in unserer Analyse 102,62 Monate (8,5 Jahre). Verglichen mit anderen Studien ist dieser Zeitraum sehr lange. Es sei jedoch darauf hingewiesen, dass die Dauer des Beobachtungszeitraums nicht der Dauer der Therapie mit SAA entspricht, sondern sich auf die Beobachtung des jeweiligen Patienten unter jeglicher Therapie bezieht beziehungsweise auch zu den Zeitpunkten fortgeführt wurde, zu denen keine Therapie stattfand. Vergleichbare Studien beschreiben oft nur den Zeitraum, in dem die Patienten mit SAA behandelt wurden und brechen ihre Beobachtung zum Zeitpunkt des Absetzens der Medikation mit SAA ab. So sind viele Studien auf einen Zeitraum von drei (Arnold et al 1996; Wang 
et al. 2006), sechs (Rinke et al. 2009) oder zwölf Monaten (Eriksson et al. 1997) angesetzt. Durch den langen Zeitraum unserer Beobachtung sei auch das Ausscheiden vieler unserer Patienten erklärt: Bei NETs handelt es sich um eine recht seltene Tumorentität. Patienten mit diesem Krankheitsbild werden demnach häufig nur in großen Tumorzentren behandelt. Ein solches Zentrum stellt die Universitätsmedizin Göttingen dar. Bei lange anhaltender Therapie jedoch werden häufig Einstellung und Anbehandlung mit einem Medikament im jeweiligen Zentrum durchgeführt und die weitere Behandlung in die Hände einer Klinik in Nähe des Wohnorts des Patienten gegeben. Dies hat zur Folge, dass Patienten nach der Phase der Anbehandlung in den Zentren nicht wieder gesehen werden, womit nur ein Teil ihrer Krankheitsgeschichte dort dokumentiert ist. Des Weiteren werden viele Patienten durch die Krankheit selbst und durch die Nebenwirkungen der Therapie, vor allem, wenn es sich um eine Chemotherapie handelt, hilfsbedürftig. Oft leben sie jedoch nicht in der Nähe von Verwandten, was zur Folge hat, dass diese Patienten während der Therapie umziehen und somit ihre weitere Behandlung in die Hände einer anderen Klinik in der Nähe ihres neuen Wohnorts legen. Auch kommt es vor, dass Patienten eine Therapie eigenmächtig abbrechen. Die Gründe für diese Entscheidung sind vielschichtig. Einer davon mag die hohe Belastung durch Nebenwirkungen vor allem bei einer Chemotherapie sein. Auch von diesen Patienten ist die Dokumentation der Krankheitsgeschichte dem zu Folge nur teilweise vorhanden.

\subsection{Therapie mit SAA}

Die Effekte, die sich in Symptomkontrolle, Hormonsekretion und Tumorwachstum durch die Therapie mit SAA in unserer Analyse gezeigt haben, sollen nun hauptsächlich mit drei Studien verglichen werden: Arnold et al. (1996), Ducreux et al. (2000) und Rinke et al. (2009). Vor der Gegenüberstellung der jeweiligen Ergebnisse soll daher erst einmal ein Überblick über diese Studien gegeben werden:

Die in Deutschland durchgeführte multizentrische prospektive Phase-II-Studie von Arnold et al. (1996) beschreibt die Effekte einer Therapie mit Octreotid bei Patienten mit metastasierten NETs. Beschrieben werden der Verlauf der Symptomatik, der Tumormarker und des Tumorwachstums jeweils nach drei und zwölf Monaten. Für den Verlauf der Symptomatik ergab sich nach zwölf Monaten folgende Verteilung: Von 20 Patienten, die zu Beginn der Therapie unter Diarrhö gelitten hatten, zeigten neun Patienten (45\%) eine Normalisierung, acht Patienten (40\%) eine Abnahme um mehr als $50 \%$ und zwei Patienten (10\%) eine Zunahme der Symptomatik. Bei einem Patienten (5\%) blieb die Symptomatik unverändert. Von 27 Patienten, die zu Beginn der Therapie unter Flush gelitten hatten, zeigten zwölf Patienten (44,4 \%) 
eine Normalisierung, elf Patienten $(40,8 \%$ ) eine Abnahme um mehr als $50 \%$ und ein Patient $(3,7 \%)$ eine Zunahme der Symptomatik. Bei drei Patienten $(11,1 \%)$ blieb die Symptomatik unverändert. Die Beobachtungen zum Verlauf der Tumormarker ergaben nach zwölf Monaten eine Normalisierung bei zwei Patienten (5,1\%), eine Abnahme um mehr als $50 \%$ bei elf Patienten $(28,8 \%)$ und eine Zunahme bei elf Patienten (28,8\%). Bei weiteren 15 Patienten $(38,5 \%)$ konnte keine Veränderung der Tumormarker beobachtet werden. Hinsichtlich des Verlaufs der Tumorgröße nach zwölf Monaten zeigten sich eine Progression bei 18 Patienten $(34,6 \%)$ und eine stable disease bei zwölf Patienten (23,1\%). Eine Regression konnte bei keinem der Patienten beobachtet werden, 22 Patienten (42,3 \%) starben während des Beobachtungszeitraums. Auffällig hierbei ist, dass die höchste Rate der Progressionen und Todesfälle unter den Patienten zu beobachten war, deren Tumoren schon vor Beginn einer Therapie mit Octreotid oder während den ersten drei Monaten der Beobachtung ein progressives Wachstum zeigten. Der größte Anteil der Patienten in der stable disease ist hingegen unter denjenigen zu suchen, die schon während der ersten drei Monate eine Stabilisation des Tumorwachstums aufgewiesen hatten. Gleichzeitig zeigt sich unter diesen Patienten der niedrigste Anteil an Progression oder Todesfällen. Die durchschnittliche Dauer der stable disease betrug 18 Monate.

Die von Ducreux et al. (2000) durchgeführte prospektive Phase-II-Studie untersucht die Wirkung von Lanreotid. Hierfür wurden Primärtumoren jeder Lokalisation unabhängig von ihrem Metastasierungsstatus berücksichtigt. Von 38 Patienten zeigten 15 (40\%) eine Normalisierung der Flush-Symptomatik nach zwölf Monaten. Zur Beurteilung des Verlaufs der Tumormarker wurden die Werte von Serotonin und 5-HIES beobachtet: Für Serotonin, das bei 36 Patienten gemessen wurde, zeigten 13 Patienten (36,1\%) einen Rückgang um mehr als $30 \%$, elf Patienten (30,1\%) eine Zunahme um mehr als $30 \%$ und zwölf Patienten (33,3\%) einen unveränderten Serumspiegel. 5-HIES wurde bei 37 Patienten gemessen, von denen bei 15 (40,5\%) eine Abnahme um mehr als $30 \%$, bei 13 (35,1\%) eine Zunahme um mehr als $30 \%$ und bei neun (24,3\%) keine Veränderung zu beobachten war. Was das Tumorwachstum unter Lanreotid betrifft, so konnte bei 16 Patienten (42,1 \%) eine Progression, bei 19 Patienten (50\%) eine stable disease und bei zwei Patienten (5,3\%) eine Regression festgestellt werden. Die durchschnittliche Dauer der stable disease betrug 9,5 Monate.

Die PROMID-Studie von Rinke et al. (2009) stellt die erste und bisher einzige doppelblinde, Placebo-kontrollierte und randomisierte Studie auf dem Gebiet der antiproliferativen Wirkung von SAA dar. Im Gegensatz zu der Studie von Arnold et al. (1996) wurde in dieser Studie das lang-wirksame Octreotid LAR eingesetzt. Das Patientenkollektiv wurde auf Patienten, die 
unter einem metastasierten NET des Midgut (Ileum, Zökum, Colon ascendens, Appendix) litten, begrenzt. Der Beobachtungszeitraum dieser Studie bezieht sich auf eine Therapiedauer von sechs Monaten. Es zeigte sich eine Kontrolle der Flush-Symptomatik bei sieben von zehn Patienten (70 \%) in der Octreotid LAR-Gruppe gegenüber drei von zwölf Patienten (25\%) in der Placebo-Gruppe und eine Kontrolle der Diarrhö bei zwei von sieben Patienten $(28,6 \%)$ in der Octreotid LAR-Gruppe gegenüber einem von sechs Patienten $(16,7 \%)$ in der PlaceboGruppe. In der Octreotid LAR-Gruppe wiesen 26 Patienten zu Beginn der Studie einen erhöhten CgA-Spiegel auf, in der Placebo-Gruppe waren es 30 Patienten. Im Beobachtungszeitraum kam es bei neun Patienten (34,6 \%) der Octreotid LAR-Gruppe und bei vier Patienten (13,3 $\%)$ der Placebo-Gruppe zu einer Normalisierung der Serumwerte. Zur Beurteilung des Tumorwachstums wurden 42 Patienten der Octreotid LAR-Gruppe und 43 Patienten der Placebo-Gruppe beobachtet: Unter Octreotid zeigte sich eine Tumorprogression bei zehn Patienten $(23,8 \%)$, eine Regression bei einem Patienten $(2,4 \%)$ und eine stable disease bei 28 Patienten $(66,7 \%)$. In dieser Gruppe waren sieben Todesfälle $(16,7 \%)$ zu verzeichnen. Unter Placebo konnte bei 23 Patienten (53,3\%) eine Progression, bei einem Patienten (2,3\%) eine Regression und bei 16 Patienten 37,2 \%) eine stable disease beobachtet werden. In dieser Gruppe starben neun Patienten (20,1\%) während des Beobachtungszeitraums. Die durchschnittliche Dauer der stable disease betrug unter Octreotid LAR 14,3 Monate und unter Placebo 6 Monate.

Vergleicht man das Studiendesign der drei oben aufgeführten Studien mit der unseren Analyse, aber auch untereinander, so werden einige Unterschiede deutlich: Lokalisation des Primärtumors, Stadium der Metastasierung zu Beginn der Therapie, Art und Applikation des Präparates und Beobachtungszeitraum. In unserer Analyse wurde im Gegensatz zu den oben genannten Studien nicht zwischen den verschiedenen Präparaten und deren Applikationsarten unterschieden. Auch wurden Primärtumoren jeder Lokalisation unabhängig vom Metastasierungsstadium berücksichtigt. Da die Daten unserer Analyse unter anderem nahe legen, dass die Dauer der stable disease unter der Therapie mit SAA nicht vom Metastasierungsstadium abhängt, ist eine Unterscheidung bezüglich der Effekte einer Therapie hinsichtlich des Vergleichs von Patienten, die bei Erstdiagnose schon Metastasen aufwiesen und solchen, bei denen zu diesem Zeitpunkt noch keine Metastasen nachgewiesen werden konnten, auch nicht notwendig. Diese Erkenntnis macht zugleich einen Vergleich zwischen Studien, die zwischen diesen Patientenkollektiven unterscheiden und solchen, die dies nicht tun, möglich.

Zum allgemeinen Vergleich der Verläufe untereinander sei zudem angemerkt, dass bei einem Teil der retrospektiv analysierten Patienten die derzeit angewendeten Kriterien zur Beurtei- 
lung des Verlaufs keine Anwendung gefunden haben und daher nicht erhoben werden können, da sie nach damaligem Kenntnisstand nicht untersucht worden waren. Anhand von Messdaten und Laborwerten wurden die aktuell gültigen Kriterien daher während der Erhebung dieser Daten nachträglich angewandt. Dies hat dennoch das Fehlen einzelner Daten zu Folge.

\subsubsection{Dosierung der SAA}

Patienten, die in der Universitätsmedizin Göttingen aufgrund eines NET eine Therapie mit SAA erhielten, wurden in aller Regel zunächst mit einer Dosis von $50 \mu \mathrm{g}$ dreimal täglich subkutan behandelt. Je nach Kontrolle der Symptomatik wurde die Dosis im Laufe der Zeit auf bis zu 30 mg einmal monatlich intramuskulär gesteigert. Dies entspricht somit einer Steigerung der Anfangs-Tagesdosis von $150 \mu \mathrm{g}$ auf eine End-Tagesdosis von $1000 \mu \mathrm{g}$ bei Progression (nach RECIST). Nicht alle Patienten wurden jedoch von subkutan auf intramuskulär umgestellt. Bei einigen der Patienten wurde auch die dreimalige Gabe subkutan beibehalten und auf eine jeweilige Einzeldosis von bis zu $300 \mu \mathrm{g}$ gesteigert. Dosis und Applikationsart sollen im Folgenden diskutiert werden:

Rubin et al. (1999) führten eine doppelblind-Studie zu Octreotid LAR in drei verschiedenen Dosierungen verglichen untereinander und mit Octreotid subkutan dreimal täglich durch. 79 Patienten wurden dabei in vier Gruppen eingeteilt: Eine erste Gruppe, deren Patienten dreimal täglich 0,3 - 0,9 mg Octreotid subkutan verabreicht bekam, eine zweite Gruppe, deren Patienten einmal monatlich $10 \mathrm{mg}$ Octreotid LAR erhielten, eine dritte und vierte Gruppe, deren Patienten mit jeweils 20 mg beziehungsweise 30 mg Octreotid LAR einmal monatlich behandelt wurden. Über einen Zeitraum von sechs Monaten wurden die Patienten bezüglich allgemeinem Behandlungserfolg, Symptomkontrolle und 5-HIAA-Wetren im Urin beobachtet. Bezüglich des allgemeinen Behandlungserfolgs war kein signifikanter Unterschied zwischen den vier Gruppen zu sehen. Auch was die Kontrolle der Diarrhö betraf, konnten die Ergebnisse keinen Unterschied zwischen den Gruppen zeigen. Lediglich in der Kontrolle des Flushs und bei der Normalisierung der Werte für 5-HIAA im Urin war die Gabe von 10 mg Octreotid LAR den anderen Gruppen minimal unterlegen. Eine Korrelation zwischen Steigerung der Dosis und Ausprägung der Nebenwirkungen konnte nicht bestätigt werden. Insgesamt konnte diese Studie also zeigen, dass zwischen der Applikationsart und der zu beobachtenden Wirkung keine Korrelation besteht. Des Weiteren kann anhand dieser Studie die Vermutung geäußert werden, dass eine höhere Dosis nicht immer einen höheren Nutzen hat und daher eine individuelle Entscheidung für den jeweiligen Patienten getroffen werden muss. Diese Vorgehensweise kann an der sehr unterschiedlich, individuell auftitrierten Dosis und anhand der von 
Patient zu Patient angepassten Applikationsart der SAA im Patientenkollektiv der vorliegenden Analyse beobachtet werden.

Eine wichtige Beobachtung der Studie von Rubin et al. (1999) besagt außerdem, dass ein Steady State des Medikamentenspiegels schneller mit der täglich dreimaligen subkutanen Gabe zu erreichen ist. Eine Aufdosierung mit SAA sollte also bevorzugt per subkutaner Gabe dreimal täglich durchgeführt werden, bevor eine Umstellung auf ein Monatspräparat erfolgt. Alle Patienten des Kollektivs dieser Analyse waren zu Beginn dreimal täglich subkutan therapiert worden, bevor die Dosis nach und nach anhand der Symptomatik auftitriert wurde und die Entscheidung je nach Patient auf ein Monatspräparat fiel. Dieses Vorgehen entspricht also den Empfehlungen, die sich aus der oben vorgestellte Studie ableiten lassen.

Auch die Phase-III-Studie von Bajetta et al. (2006), die eine Gabe von Lanreotid Autogel $120 \mathrm{mg}$ alle sechs Wochen mit der Gabe von Lanreotid Mikropartikeln $60 \mathrm{mg}$ alle drei Wochen an insgesamt 60 Patienten verglich, zeigt ähnliche Ergebnisse: Auch hier konnte den verschiedenen Präparaten und Applikations-Häufigkeiten eine gleichwertige Effektivität bezüglich der Kontrolle von Tumormarkern und Tumorgröße bei NETs zugeschrieben werden.

\subsubsection{Therapiedauer}

Verglichen mit den drei zuvor beschriebenen Studien (siehe Kapitel 4.3), die dieser Diskussion zugrunde liegen (Arnold et al. 1996; Ducreux et al. 2000; Rinke et al 2009), zeigte sich in unserer Analyse eine sehr lange Beobachtungsdauer von 29 Monaten. In der Studie von Ducreux et al. (2000) betrug die durchschnittliche Therapiedauer lediglich 9,5 Monate. Patienten unserer Analyse wurden jedoch währen ihrer Therapie in einem nicht limitierten Zeitraum beobachtet (zur Definition des Beobachtungszeitraums siehe auch Kapitel 4.2). Daher rührt auch die im Vergleich zu anderen Studien lange maximale Therapiedauer von 213 Monaten unserer Beobachtung. Diese lange Therapiedauer ist per se schon ein Indiz für die Wirkung, die eine Therapie mit SAA auf eine Stabilisierung des Tumorwachstums hat. Gleichzeitig liefert sie ein Indiz für die gute Verträglichkeit von SAA, die von mehreren Autoren postuliert wird (Kulke und Mayer 1999; Rubin 1999) und auf die bereits in Kapitel 1.3.2 eingegangen wurde. Über das Auftreten von Nebenwirkungen liegen in unserer Analyse jedoch keine Daten vor.

\subsubsection{Verlauf der Symptomatik}

Daten zum Verlauf der Symptomatik beruhen auf Patientenbefragungen zu den regelmäßigen Terminen der Verlaufskontrolle (siehe auch Kapitel 2.3). Bewertet wurde anschließend der 
gesamte Verlauf während der Zeiträume mit vs. ohne SAA, wobei kein separater Vergleich der Symptomatik nach definierten Zeitspannen erfolgte.

In unserer Beobachtung ergab sich ein großer Anteil an Patienten (73\%), deren Symptomatik (Flush und/oder Diarrhö) sich unter der Therapie nicht veränderte. Was diesen Anteil betrifft, so muss, vor allem bezüglich der Diarrhö, die Art der Datenerhebung beachtet werden. Die dafür verwendeten Daten unserer Analyse basieren auf Patientenbefragungen und der Messung des spezifischen Stuhlgewichts. Oft kann jedoch nicht unterschieden werden, welchen Ursprungs die gezeigte Symptomatik ist. Vor allem Diarrhö muss nicht immer die Folge der durch den Tumor freigesetzten Hormone sein. Viele der Patienten, die unter einem NET leiden, erfahren primär eine operative Therapie. Im Zuge dieser Operationen werden oft große Teile des Darms entfernt, was nicht selten zum Verlust von Gallensäure oder zu einem Malabsorptionssyndrom führt. In manchen Fällen ist auch eine Entfernung der Ileozökalklappe erforderlich, was zu einer vermehrten bakteriellen Besiedlung des Dünndarms führen kann. Diese drei hier aufgeführten Pathomechanismen können jeweils unabhängig voneinander zur Symptomatik einer Diarrhö führen, ohne dass diese auf die vom Tumor produzierten Hormone zurückzuführen wäre. Es ist also nicht ausgeschlossen, dass die Therapie mit SAA in unserem Patientenkollektiv durchaus bei dem einen oder anderen Patienten zu einem positiven Effekt bezüglich der Diarrhö führte, dieser Effekt aber durch die dennoch gezeigte Symptomatik als Folge anderer Pathomechanismen verschleiert wurde. Des Weiteren muss darauf hingewiesen werden, dass es sich hier um einen sehr langen Zeitraum der Beobachtung (in einzelnen Fällen über Jahre, siehe Kapitel 3.3.2) handelt. Denkbar ist daher, dass sich auf einen primären Therapieerfolg im Sinne einer Symptombesserung eine erneute Verschlechterung (beispielsweise aufgrund einer Tachyphylaxie, vergleiche Kapitel 4.3.4) einstellte. Im Langzeitverlauf ist dies dann als insgesamt fehlende Änderung der Symptomatik zu verzeichnen, obwohl eine Therapie mit SAA nicht primär erfolglos blieb.

Im Folgenden soll nun zuerst auf die Symptomatik im Allgemeinen (Flush und/oder Diarrhö) eingegangen werden: Betrachtet man den Verlauf der Symptomatik unter SAA bei den $27 \%$ der Patienten, die im Beobachtungszeitraum eine Änderung der Symptomatik vorwiesen, so steht der Anteil der Patienten, deren Symptomatik unter der Therapie abnahm im Vergleich zu denjenigen Patienten, die unter SAA eine Steigerung der Symptomatik beschrieben im Verhältnis von 2:1. Der Anteil von 17,8 \% an Patienten, deren Symptomatik im beobachteten Zeitraum abnahm, deckt sich mit der Studie von Rinke et al. (2009), in der sich für diese Patientengruppe ein Anteil von 21,2 \% zeigte. Ducreux et al. (2000) beschrieben in ihrer Studie einen Anteil von $40 \%$ an abnehmender Symptomatik. In einer Studie von Garland et al. 
(2003) konnte sogar ein Anteil von 85,2 \% beobachtet werden. Betrachtete die Studie von Rinke et al. (2009) einen Zeitraum von sechs Monaten und Ducreux et al. (2000) eine Zeitraum von einem Jahr, so betrug die mittlere Beobachtungsdauer in der Studie von Garland et al. (2003) 23 Monate. Dies lässt vermuten, dass die Anzahl der Patienten, die bezüglich der Symptomatik einen positiven Effekt der Therapie nachweisen können, mit der Zeit der Therapiedauer zunimmt. Im Patientenkollektiv unserer Analyse wiesen vier der sieben Patienten, die ein positives Ansprechen auf SAA zeigten eine Behandlungsdauer von 29 Monaten und mehr auf (29 Monate, 34 Monate, 58 Monate und 68 Monate). Schaut man sich die von Arnold et al. (1996) durchgeführte Studie an, so könnte diese Vermutung bestätigt werden: Arnold et al. (1996) beobachteten und bewerteten den Verlauf der Symptomatik nach jeweils drei und zwölf Monaten. Nach zwölf Monaten fiel auch in dieser Studie ein deutlich überwiegender Anteil der Patienten, bei denen die Therapie einen positiven Effekt auf die Symptomatik hatte, auf (85\%). Auffällig ist hierbei der Vergleich der gezeigten Symptomatik nach einer Therapiedauer von drei Monaten mit der Symptomatik, die nach zwölf Monaten zu beobachten war: Im Zeitraum zwischen dem dritten und zwölften Therapiemonat konnte unter den Patienten, deren Symptomatik unter der Therapie zunahm oder unverändert blieb, eine Abnahme um bis zu $58 \%$ beobachtet werden. Gleichzeitig stieg der Anteil der Patienten, deren Symptomatik abnahm um $130 \%$ an. Auch in der Studie von Arnold et al. (1996) zeigt sich also - wie in unserer Beobachtung - ein wesentlich größerer Anteil an Patienten, deren Symptomatik rückläufig war im Vergleich zu denjenigen, die eine Progredienz zeigten.

Bezogen auf die zu Beginn der Therapie gezeigte Symptomatik konnte in unserer Analyse beobachtet werden, dass eine Therapie mit SAA vor allem dann wirksam ist, wenn bei Therapiebeginn sowohl Diarrhö als auch Flush vorlagen. Dieses Ergebnis ist umso wichtiger, da der weitaus größte Teil der Patienten, die unter einer Karzinoidsymptomatik leiden, sowohl Diarrhö, als auch Flush-Symptomatik aufweisen. Somit ist die Wahrscheinlichkeit, dass dieser große Anteil der Patienten von einer symptomkontrollierenden Therapie mit SAA profitiert also sehr hoch. Das überwiegende Verhältnis der Patienten, die unter dieser kombinierten Symptomatik leiden, zeigte sich auch in unserer Analyse: Unter den Patienten mit funktionellem Tumor, die zu Therapiebeginn eine Symptomatik aufwiesen, litten 65,8\% unter Flush und Diarrhö. Diese Angaben decken sich mit dem Patientenkollektiv der Studie von Garland et al. (2003). Hier zeigten 69,2 \% der Patienten zu Therapiebeginn sowohl Flush als auch Diarrhö. Allerdings konnte ein Unterschied im Ansprechen der Therapie je nach zu Beginn der Therapie gezeigter Symptomatik dort nicht nachgewiesen werden. 
Es lässt sich also schlussfolgern, dass SAA einen positiven Effekt auf den Verlauf der Symptome, welche durch die vom Tumor produzierten Hormone verursachten werden, haben. Am meisten profitieren Patienten mit schwerwiegender Symptomatik, also Patienten, die unter Flush und Diarrhö leiden. Dieses Patientenkollektiv kann durch eine Therapie mit SAA eine wesentliche Steigerung der Lebensqualität erfahren. Effekte auf weitere Symptome des Karzinoid-Syndroms (Bauchschmerzen, Teleangiektasien, Bronchokonstriktion, Ödeme oder Karzinoid-Herzschäden) wurden in dieser Analyse nicht untersucht.

\subsubsection{Verlauf der Tumormarker}

Nach Kaltsas et al. (2002) kann der Verlauf der Tumormarker in folgende Untergruppen eingeteilt werden: Kein Ansprechen, partielles Ansprechen, komplettes Ansprechen und Progression (Vergleiche auch Kapitel 2.3.1). Diese Einteilung wurde auch der Auswertung der vorliegenden Daten zugrunde gelegt. Dabei beobachtet wird der gesamte Zeitraum der Therapie mit SAA, unabhängig von Präparat und Dosis. In den meisten Fällen fand auch während dieses Zeitraums eine Dosissteigerung statt (vergleiche Kapitel 4.3.1). Von einem fehlenden Ansprechen wird dann gesprochen, wenn die Werte der Tumormarker weder eine wesentliche $\mathrm{Zu}$ - noch eine wesentliche Abnahme vorweisen. Eine dermaßen stabile Situation der Tumormarker darf jedoch nicht mit dem Verlauf der Tumorgröße und einer stable disease gleichgesetzt werden. In einer von Wang et al. (2006) hierzu durchgeführten Studie konnte beobachtet werden, dass auch bei hormonellem Nichtansprechen ein Ansprechen der Therapie bezüglich des Tumorwachstums vorliegen kann. Auch Kölby et al. (2003) postulierten, das aufgrund der Ergebnisse ihrer Studie keine Korrelation von Tumormarker zu Überlebensrate oder Risiko einer Progression des Tumorwachstums gemacht werden kann.

Stellt man den Vergleich des Verlaufs der Tumormarker unter einer Therapie mit SAA mit dem Zeitraum, in dem keine Therapie mit SAA stattfand auf der Basis der vorliegenden Daten unserer Analyse an, so können folgende Beobachtungen gemacht werden: Der Anteil der Patienten, die auf die Therapie kein Ansprechen zeigten (30\%), stimmt mit dem Anteil entsprechender Patienten in der Studie von Ducreux et al. (2000) überein. Dort wurde dieser Patientenanteil mit 28,7 \% beschrieben. Eine hohe Konzentration von CgA hat, was das Überleben, aber auch die Chancen auf ein Ansprechen auf eine Therapie mit SAA betrifft, einen negativen Einfluss auf die Prognose (Janson et al. 1997). Je höher also die Konzentration an CgA, desto wahrscheinlicher das Nicht-Ansprechen auf die Therapie beziehungsweise eine Progression der Tumormarker unter Therapie. In den teils initial stark erhöhten CgA-Werten unseres Patientenkollektivs (bei sechs der beobachteten Patienten um mehr als das Drei- bis Achtfache, bei vier der beobachteten Patienten um mehr als das Zehnfache der oberen Normgrenze) 
mag somit der hohe Anteil der Patienten, die trotz SAA einen schlechten Verlauf zeigten, begründet sein: In unserer Analyse zeigten vier der Patienten mit initial stark erhöhten CgAWerten ein Nicht-Ansprechen, fünf dieser Patienten zeigten eine Progression. Garland et al. (2003) beschrieben in ihrer Studie einen Anteil an partiellem Ansprechen von 14,8\%. Auch dieser Anteil lässt sich mit den Ergebnissen der vorliegenden Analyse (hier $9 \%$ ) vereinbaren. Bezogen auf die Rate des kompletten Ansprechens sind unsere Daten (9\%) mit den Daten der Studie von Garland et al. (2003) (7,4\%) zu vergleichen. Vergleicht man unsere Ergebnisse jedoch mit der Studie von Rinke et al. (2009), so fällt eine weitaus höhere Rate an komplettem Ansprechen (34,6 \%) bei Rinke et al. (2009) auf. Hierzu anzumerken ist jedoch, dass die Konzentration von 5-HIES im 24-Stunden-Urin für die Verlaufskontrolle von NETs des Midgut ein sinnvoller Parameter ist, sich zur Beurteilung von Tumoren des Foregut und Hindgut jedoch als weniger gut geeignet erwiesen hat (Janson et al. 1997). Rinke et al. (2009) betrachteten ausschließlich NET, die unter die Kategorie der Midgut fielen, wohingegen die Patienten unseres Kollektivs NETs verschiedenster Kategorien aufwiesen (Foregut, Midgut und Hindgut). Hierin mag der Grund der weit höheren Rate eines kompletten Ansprechens bei Rinke et al. (2009) liegen. Die Studie von Ducreux et al. (2000) ergab mit 32,7 \% einen recht großen Anteil an progredient verlaufenden Erkrankungen, was mit den $42 \%$ progredienter Verläufe unserer Beobachtungen vergleichbar ist. Allerdings muss hierzu angemerkt werden, dass die Auswertungskriterien nach Kaltsas et al. (2002) in der Studie von Ducreux et al. (2000) nicht angewendet wurden. Sprechen Kaltsas et al. (2002) bei einer Zunehmen der Tumormarker um $25 \%$ des Ausgangswertes schon von einer Progression, so definieren Ducreux et al. (2000) diese erst ab einer Zunahme von mehr als $30 \%$. Dies mag den unterschiedlichen Anteil der Progressionen erklären.

Vergleicht man nun den Verlauf der Tumormarker im Zeitraum mit und ohne SAA innerhalb der Patienten unserer Analyse, ergibt sich eine Abnahme der Patienten, die kein Ansprechen aufwiesen um $33 \%$ (30/66 Patienten im Zeitraum ohne SAA vs. 20/66 Patienten im Zeitraum mit SAA) und eine Abnahme des partiellen Ansprechens um 14 \% (7/66 Patienten im Zeitraum ohne SAA vs. 6/66 Patienten im Zeitraum mit SAA) unter SAA. Gleichzeitig steigt die Zahl der Patienten mit einem kompletten Ansprechen um das Doppelte (3/66 Patienten im Zeitraum ohne SAA vs. 6/66 Patienten im Zeitraum mit SAA). Es steigt jedoch auch die Zahl der progredient verlaufenden Erkrankungen um 40 \% (20/66 Patienten im Zeitraum ohne SAA vs. 28/66 Patienten im Zeitraum mit SAA). (Daten zu den hier genannten Zahlen siehe Kapitel 3.3.5, Abbildung 9) Für den steigenden Anteil der Progressionen unter Therapie mit SAA könnte die Tachyphylaxie eine Erklärung liefern: 
Hofland und Lamberts (2003) beschrieben anhand der Konzentration von 5-HIES im 24Studnen-Urin eines Patienten, wie sich die Tachyphylaxie auf den Verlauf dieses Tumormarkers auswirken kann: War es bei diesem Patienten in den ersten drei Monaten zu einer deutlichen Abnahme des HIES-Werts um mehr als $50 \%$ gekommen, so stieg dessen Wert ab dem vierten Monat des Beobachtungszeitraums wieder kontinuierlich an und überschritt die ursprüngliche Konzentration, die bei Therapiebeginn vorgelegen hatte. So war nach 21 Monaten ein Maximum erreicht, das nunmehr das Doppelte der zu Therapiebeginn gemessenen Konzentration betrug. Betrachtet man in diesem Fall den gesamten Zeitraum der Therapie, müsste trotz eines anfänglich sehr guten Therapieerfolgs von einer progredient verlaufenden Erkrankung gesprochen werden. Demzufolge ist die Gefahr, dass der Anteil der progredient verlaufenden Erkrankungen durch die induzierte Tachyphylaxie gesteigert wird, umso größer, je länger man den Zeitraum der Beobachtung wählt. Dieser Sachverhalt wird durch die Studie von Arnold et al. (1996) bestätigt. Hier wurde die Konzentration der Tumormarker nach jeweils drei und zwölf Monaten gemessen. Der Anteil der Patienten mit einem kompletten Ansprechen nahm dabei zwischen den Messungen nach drei Monaten und den Messungen nach zwölf Monaten von 11,5 \% auf 5,1 \% ab. Dies entspricht einer Abnahme um 55,6 \%. Gleichzeitig nahm die Anzahl der progredient verlaufenden Erkrankungen in diesem Zeitraum von $24,6 \%$ auf 28,2 \% zu, was einer Steigerung um fast $20 \%$ entspricht. Die Zunahme der progredient verlaufenden Erkrankungen ist also auch in der Studie von Arnold et al. (1996) zu beobachten und lässt sich somit mit der Zunahme der Progressionen der vorliegenden Analyse vereinbaren.

Im Folgenden soll nun geschaut werden, welche Effekte eine Monotherapie mit SAA, verglichen mit SAA plus Zusatztherapie, hat. Unter Zusatztherapie zählen in dieser Analyse jede Art von Chemotherapie, eine Therapie mit Interferon- $\alpha$ sowie eine nuklearmedizinische Therapie. Für den Vergleich des Patientenkollektivs, das eine zusätzliche Therapie erhielt und des Kollektivs, bei welchem eine Monotherapie mit SAA durchgeführt wurde, ergeben sich folgende Verhältnisse: Im Anteil der Patienten, die kein Ansprechen oder ein partielles Ansprechen aufwiesen, konnte kein wesentlicher Unterschied der beiden Gruppen festgestellt werden. Die Anzahl der Patienten mit komplettem Ansprechen stieg um über das Fünffache in der Gruppe, die eine Zusatztherapie erhalten hatte (1/66 Patienten unter SAA-Monotherapie vs. 5/66 Patienten mit SAA plus Zusatztherapie). Die progressiv verlaufenden Erkrankungen reduzierten sich um 40 \% (19/66 Patienten unter SAA-Monotherapie vs. 9/66 Patienten mit SAA plus Zusatztherapie). (Vergleiche Kapitel 3.3.5, Abbildung 13) Für diesen Vergleich ergab sich jedoch keine statistische Signifikanz. Es kann also keine allgemeine Aussage über 
den zusätzlichen Nutzen oder Schaden einer Zusatztherapie gemacht werden. Dennoch wird diese Aussage auch von der Studie von Kölby et al. (2003) unterstützt: Verglichen wurden die Effekte einer alleinigen Therapie mit Octreotid bei 35 Patienten und einer Kombinationstherapie aus Octreotid und Interferon- $\alpha$ bei 33 Patienten. In der Auswertung der 5-HIES-Werte im 24-Stunden-Urin konnte bei Kölby et al. (2003) kein signifikanter Unterschied zwischen den Patienten mit und den Patienten ohne Zusatztherapie beobachtet werden. Dennoch kann in unserem Kollektiv eine Tendenz hinsichtlich eines geringen Mehrnutzens einer zusätzlichen Therapie gesehen werden. Da das Patientenkollektiv insgesamt recht klein ist und sich damit unter der Aufteilung bestimmter Untergruppen eine noch kleinere Stichprobenzahl ergibt, wären zukünftige Studien hierüber allerdings nötig.

Der Vergleich der verschiedenen Tumormarker untereinander gestaltet sich schwierig, da sie sich in ihrer Sensitivität und Spezifität wesentlich unterscheiden. CgA ist der sensitivste von ihnen und gilt deshalb heute als Parameter der ersten Wahl. Dennoch liegt seine Entdeckung noch nicht allzu lange zurück, weswegen für Patienten, deren Daten aus früher beobachteten Zeiträumen stammen, keine Werte zur Verfügung stehen. Außerdem weisen verschiedene Tumormarker je nach Sekretionsverhalten des zugrunde liegenden Tumors unterschiedliche Konzentrationen auf: CgA kann bei funktionellen und nicht-funktionellen Tumoren nachgewiesen werden, die Konzentration von 5-HIES im 24-Stunden-Urin hingegen steigt nur bei bestimmten Gruppen von metastasierten NETs. Liegt ein nicht-funktioneller Tumor vor oder befindet sich dieser noch im nicht-metastasierten Stadium, so ist der 5-HIES-Wert negativ (O’Toole et al. 2009). Dies kommt vor allem bei im Midgut lokalisierten Primärtumoren vor (Garland et al. 2003). NSE wiederum ist ein zusätzlicher Marker bei schlecht-differenzierten NECs (Öberg et al. 1999), weswegen er nur in dem Anteil der Patienten bestimmt wurde, die an solch einem Tumor erkrankt waren. Zudem können die Werte der Tumormarker durch exogene und endogene Einflüsse in ihrer Konzentration verändert werden, was zu falschnegativen oder falsch-positiven Werten führen kann. Besonders anfällig hierfür sind $\mathrm{CgA}$ und 5-HIES. (Zur Charakterisierung der Tumormarker vergleiche auch Kapitel 1.2.3.)

Schlussfolgernd lässt sich trotz der schlechten Vergleichbarkeit sagen, dass auch die Daten der vorliegenden Analyse einen positiven Effekt von SAA auf den Verlauf der Tumormarker bei Patienten mit NET bestätigt haben: Der Anteil der Patienten, die unter der Therapie mit SAA keine Progression erlitten, liegt hier im wie in Kapitel 3.3.2 beschriebenen Zeitraum der Gesamtdauer der Therapie mit SAA bei $58 \%$. 


\subsubsection{Stable disease unter SAA}

Wie in Kapitel 2.3.2 beschrieben, richtet sich die Beurteilung der stable disease nach dem Verlauf der Tumorgröße und nach dem Auftreten neuer Metastasen (vgl. RECIST-Kriterien). Im folgenden Kapitel beinhaltet der Begriff „stable disease“ also immer sowohl den Verlauf der Tumorgröße als auch den der Metastasen. In Kapitel 4.3.6 bis 4.3.8 wird dann auf die Verläufe von Tumorgröße und Metastasen gesondert eingegangen.

Arnold et al. (1996) hatten eine im Mittel 18 Monate lange Dauer der stable disease unter Octreotid festgestellt, Ducreux et al. (2000) wiesen dagegen unter Lanreotid lediglich eine Dauer der stable disease von durchschnittlich 9,5 Monaten nach. Unter Octreotid LAR konnte in der Studie von Rinke et al. (2009) eine mittlere Dauer der stable disease von 14,3 Monaten erreicht werden. Schon diese Unterschiede in der Literatur lassen vermuten, dass die Dauer der stable disease in Abhängigkeit des betrachteten Patientenkollektivs und der verwendeten Therapie stark variieren kann. Unsere Analyse zeigt einen längeren Beobachtungszeitraum, daher auch eine längere Dauer der stable disease von durchschnittlich 21,4 Monaten. Auch die Daten unserer Analyse lassen also vermuten, dass von einer Verlängerung der stable disease durch den Einsatz von SAA in der Therapie von NET ausgegangen werden kann. Für die Bestätigung dieser Annahme wäre eine entsprechende Studie, die den Vergleich mit einer Kontrollgruppe zieht, nötig.

Betrachtet man Patientenkollektive unter dem Gesichtspunkt einer bereits stattgehabten Metastasierung, identifizierten Arnold et al. (1996) und später auch Rinke et al. (2009) den Umfang der hepatischen Metastasierung als negativen Prognosefaktor. Verschiedene Stadien der Metastasierung wurden in unserer Analyse nicht untersucht und berücksichtigt. Allerdings lässt sich mit den Daten unserer Patientenkollektive zeigen, dass eine per se vorhandene Metastasierung zu Beginn der Therapie keine Auswirkung auf deren Erfolg im Sinne der Dauer einer stable disease hat. Der bei Erstdiagnose gezeigte Metastasierungsstatus sollte daher bezüglich der Anwendung von SAA auch nicht therapieentscheidend sein. Einen Beleg hierfür liefert der Vergleicht unserer Daten mit der Studie von Arnold et al. (1996): Der Studie von Arnold et al. (1996) liegt ein Patientenkollektiv zugrunde, in dem alle Tumoren einen bereits metastasierten Status aufwiesen. Die mittlere Dauer der stable disease betrug 18 Monate. In unserer Analyse ergab die durchschnittliche Dauer der stable disease im zum Therapiebeginn nicht metastasierten Patientenkollektiv ebenfalls 18 Monate. Das Ausmaß der Effekte durch eine Therapie mit SAA scheint also nach Betrachtung dieses Vergleichs der verschiedenen Analysen von einer initial gezeigten Metastasierung per se unabhängig zu sein, wobei der Umfang der Metastasierung wie oben erwähnt von prognostischer Bedeutung sein kann. 
Im Unterschied zu den drei oben genannten Studien wurden Patienten, die aufgrund einer Progression des Tumorwachstums oder der Symptomatik eine Zusatztherapie erhielten, nicht aus der Analyse ausgeschlossen, sondern weiterhin beobachtet. Ziel war es, herauszufinden, ob ein zusätzlicher Einsatz von Chemotherapie, Interferon- $\alpha$ oder nuklearmedizinischen Methoden einen zusätzlichen Effekt auf den Verlauf der Tumorerkrankung hat. Janson und Öberg (1993) verglichen die Kombinationstherapie von Octreotid und Interferon- $\alpha$ mit einer Monotherapie von Octreotid bei Patienten mit metastasierten NET und beobachteten eine Verlängerung der stable disease um zwei Monate in dem Kollektiv, das die Kombinationstherapie erhalten hatte. Die Ergebnisse unserer Analyse ergaben jedoch, dass zwischen einem Patientenkollektiv mit und einem Kollektiv ohne Zusatztherapie kein signifikanter Unterschied bezüglich der Dauer der stable disease besteht. Hier kann daher also vermutet werden, dass eine zusätzlich Therapie für die zytoreduktive Wirkung keinen zusätzlichen Nutzen bringt. Allerdings wurde in unserer Beobachtung nicht zwischen den verschiedenen zusätzlich angewandten Therapien unterschieden. Aussagen über den alleineigen Effekt einer zusätzlichen Verabreichung von Interferon- $\alpha$ können daher in Bezug auf das Patientenkollektiv unserer Analyse nicht gemacht werden. Angeführt sei hierzu jedoch eine Studie, die von Wang et al. (2006) veröffentlicht wurde und eine durchschnittliche Dauer der stable disease von 13,3 Monaten unter der Therapie mit Sandostatin LAR plus Interferon- $\alpha$ zeigte. Diese im Vergleich zu den anderen Studien, die auf einer Monotherapie mit SAA basieren, kurze Dauer der stable disease mag unter anderem der kleinen Probandengruppe von nur zwölf Patienten geschuldet sein. Trotzdem unterstützt sie die Theorie, dass eine zusätzlich eingesetzte Therapie zwar einen zusätzlichen Effekt auf das subjektive Wohlbefinden des Patienten haben mag (vergleiche Abbildung 14; Zur Methode der Datenerhebung hierfür siehe Kapitel 2.3), in Bezug auf die Dauer der stable disease jedoch keinen zusätzlichen Nutzen bringt.

Bezüglich einer zusätzlichen Chemotherapie ist zu beachten, dass diese, wie in Kapitel 1.3.5 bereits erwähnt, nur im Falle eines vorliegenden Tumors mit geringem Differenzierungsgrad einen Erfolg aufweist. So konnte eine Chemosensitivität nur bei schlecht-differenzierten NEC nachgewiesen werden (Mitry und Rougier 2001). Gut-differenzierte und mäßig-differenzierte NET haben eine geringe Proliferationsrate, was sie für eine Chemotherapie schwer zugänglich macht. Bewertet man also den Therapieerfolg einer eingesetzten Chemotherapie, so ist immer auch der Differenzierungsgrad des jeweiligen Tumors zu beachten, unabhängig davon, ob es sich bei der durchgeführten Chemotherapie um eine Monotherapie oder eine Zusatztherapie zum Beispiel zu bereits verabreichten SAA handelt. Für eine Untersuchung bezüglich des Nutzens einer zusätzlichen Chemotherapie ist eine Einteilung des Patientenkollektivs nach 
dem Differenzierungsgrad demnach äußerst sinnvoll. Daten unserer Analyse können dies jedoch leider nicht leisten, da für $50 \%$ der Patienten kein Differenzierungsgrad bei Erstdiagnose bekannt ist (siehe auch Kapitel 3.2.1 sowie Kapitel 4.1.2). Weitere Studien diesbezüglich sind also erforderlich.

Bei Patienten, die an der Universitätsmedizin Göttingen mit SAA behandelt wurden, fiel die Entscheidung zur Durchführung einer Chemotherapie nach gezeigter Symptomatik und deren eventueller Progredienz und/oder nach Progredienz des Tumorwachstums. Entgegen oben genannter Annahme und Empfehlung fand eine Einbeziehung des Differenzierungsgrads in diese Entscheidungen nur äußerst selten (und dann bei den Erstdiagnosen, die am wenigsten lange zurück liegen) statt.

Im Hinblick auf den Zugewinn an subjektivem Wohlbefinden ist auch das jeweilige Nebenwirkungsprofiel eines Medikaments zu beachten. Viele Patienten leiden unter einer Therapie mit Interferon- $\alpha$ an Depressionen und grippeähnlicher Symptomatik (vergleiche auch Kapitel 1.3.3). Nebenwirkungen einer Chemotherapie sind vielfältig und vom jeweiligen Präparat abhängig. Neben spezifischen Nebenwirkungen wie Kardiotoxizität (vor allem Doxorubicin) beziehungsweise Neuro- oder Ototoxizität (vor allem Cisplatin) klagen Patienten vor allem über allgemeine Nebenwirkungen wie Übelkeit, Erbrechen, Diarrhö, Alopezie und Schwächegefühl. Des Weiteren stellt die chemotherapieinduzierte Panzytopenie eine Nebenwirkung dar, die häufig mit lebensgefährlichen Komplikationen verbunden ist. Die Nutzen einer zusätzlichen Therapie sollten also auch immer gegen die dadurch entstehenden zusätzlichen Symptome abgewogen werden.

Eine recht gut verträgliche Möglichkeit der zusätzlichen Therapie stellt Dacarbazin dar. Ritzel et al. (1995) führten eine Beobachtung an sieben Patienten, die an einem metastasierten NET des Midgut litten und die sie mit Dacarbazin behandelten, durch. Bei sechs Patienten zeigte sich eine Abnahme der Metastasen oder zumindest keine Zunahme in deren Größe für durchschnittlich zehn Monate. Zusätzlich zeigte sich bei sechs Patienten eine Verbesserung der klinischen Symptomatik. Was die Verträglichkeit dieser Therapie betrifft, so konnte als einzige Nebenwirkung dieser Therapie bei zwei der Patienten eine Abnahme der Leukozyten nach zwei Wochen beobachtet werden. Zimmer et al. (2012) stellen in ihrer Arbeit zur Übersicht von Chemotherapeutika noch einmal die Nebenwirkungen von Dacarbazin dar. Demnach weist Dacarbazin ein sehr hohes emetogenes und phototoxisches Potential auf und führt nicht selten zur schon von Ritzel et al. (1995) beobachteten Myelosuppression. Eine sehr seltene, aber gefährliche Nebenwirkung stellt die Gefahr der Lebernekrose durch Lebervenenver- 
schluss dar. In unserem Patientenkollektiv wurden zeitweilig 33 Patienten (50\%) mit Dacarbazin behandelt, die meisten davon (57,5 \%) zusätzlich zur Therapie mit SAA.

\subsubsection{Verlauf der Tumorgröße}

Schaut man sich die Ergebnisse unserer Analyse wie sie in Kapitel 3.3.7 dargestellt sind an, so fällt zunächst auf, dass ein Anteil von 13,64 \% unseres Patientenkollektivs auch ohne SAA eine stable disease aufzeigte. Bei diesen Verläufen handelt es sich sämtlich um Patienten, die vor der Therapie mit SAA einen stabilen Verlauf der Tumorgröße aufwiesen, bei denen wegen zunehmender Symptomatik jedoch trotzdem zu einem gewissen Zeitpunkt eine Therapie mit SAA begonnen wurde. Dies unterstreicht die Rolle der SAA in der Symptomkontrolle eines NET (siehe auch Kapitel 4.3.3).

Auf der Basis unserer Daten soll nun der Verlauf der Tumorgröße im Zeitraum mit und im Zeitraum ohne SAA verglichen werden. Daten und Zahlen, auf denen diese Vergleiche beruhen, sind in Kapitel 3.3.7 und in Abbildung $18 \mathrm{zu}$ finden. Dieser Vergleich ergibt in allen ausgewerteten Bereichen eine Verbesserung durch die Therapie mit SAA: Unter der Therapie mit SAA stieg die Rate der Patienten, die eine stable disease aufwiesen um das 3,4-Fache (9/66 Patienten im Zeitraum ohne SAA vs. 31/66 Patienten im Zeitraum mit SAA). Konnte im Zeitraum ohne SAA keine partial response beobachtet werden, so war dies bei einem Patienten unter SAA der Fall. Die Rate der progredienten Tumorerkrankungen sank um fast ein Drittel (48/66 Patienten im Zeitraum ohne SAA vs. 33/66 Patienten im Zeitraum mit SAA). Auch bezüglich der Entwicklung von Rezidiven, wie sie in Kapitel 3.3.7 definiert sind, ergab sich aus den Daten dieser Analyse eine deutliche Verbesserung: Trat im Zeitraum ohne SAA bei neun Patienten ein Rezidiv auf, so konnte dies im Zeitraum mit SAA in keinem der beobachteten Fälle nachgewiesen werden. Ducreux et al. (2000) beschreiben in ihrer Studie einen Anteil der Patienten in der stable disease von 48,7 \%. Die absolute Prozentzahl entspricht den Ergebnissen unserer Analyse (47\%).

Arnold et al. (2000) beschrieben einen Erfolg der Wachstumskontrolle bei bis zu $50 \%$ der Patienten. Werden also nur die Parameter „stable disease“, was einer Wachstumskontrolle entspricht, und „Progression“, was für ein Versagen der Wachstumskontrolle sprechen würde, angeschaut, so ergab sich in unserer Analyse ein Anteil von $47 \%$, die während des genannten Zeitraums der Therapie mit SAA eine stable disease von im Mittel 21,44 Monaten aufwiesen (Vergleiche auch Kapitel 3.3.7). Diese Zahlen ähneln den Angaben von Arnold et al. (2000). In unserem Kollektiv ergab sich außerdem ein Patientenanteil von $50 \%$, deren Tumorerkrankung auch unter SAA progredient verlief. Diese Angaben ähneln denen aus der Studie von 
Arnold et al. (1996). Dort wurden ein Anteil der stable disease von 36,5\% und ein Anteil der progredienten Tumorerkrankungen von $57,7 \%$ beschrieben.

Vergleicht man die Zahlen unserer Analyse bezüglich des progressionsfreien Überlebens, welches den Parametern „stable disease“ und ,partial response“ entspricht, mit den Zahlen der CLARINET-Studie von Caplin et al. (2014), so zeigt sich beide Male eine deutliche Zunahme der progressionsfreien Überlebenden unter der Therapie mit SAA: 65,1 \% unter Lanreotid vs. 33,0 \% unter Placebo in der CLARINET-Studie verglichen mit 48,5\% im Zeitraum mit SAA vs. 13,6 \% im Zeitraum ohne SAA in unsere Analyse. (Zur detaillierten Beschreibung der CLARINET-Studie siehe Kapitel 1.3.2)

Auffällig ist vor allem das häufigere Auftreten einer Progression im Vergleich zur stable disease, das sowohl in der Beobachtung von Arnold et al. (1996), als auch in der unserer Analyse gesehen werden konnte. $\mathrm{Zu}$ beachten ist allerdings, dass der Anteil der stable disease bei Arnold et al. (1996) im Vergleich zu unserer Analyse wesentlich geringer ausfällt, wobei der Anteil der tumorprogredienten Patienten höher ist. Gründe dafür mögen in dem für die Aussagekraft der Bewertung unterschiedlich festgelegten Zeitraum der einzelnen Studien liegen: Arnold et al. (1996) bewerteten den Effekt der Therapie jeweils nach sechs bis zwölf Monaten. Unsere Beobachtung der Effekte erstreckt sich über den gesamten Zeitraum der Therapie, wie sie in Kapitel 3.3.2 als Gesamtdauer der Therapie mit SAA beschrieben ist (47,68 \pm 50,84 Monate. Minimum: 0 Monate, Maximum: 212 Monaten). Dies ließe zunächst vermuten, dass die Erfolgsrate einer Therapie mit SAA mit fortschreitender Therapiedauer steigt. Diese Vermutung muss jedoch durch diverse Studien widerlegt werden: Eine Studie von Aparicio et al. (2001) erbrachte eine stable disease bei 58,8 \% der Patienten nach 9 Monaten Therapiedauer. Nach 10,5 Monaten ergab sich jedoch nur noch eine Rate von 54,5\% an stable disease. Daten des progressionsfreien Überlebens über zwei Jahre in derselben Studie ergeben zudem folgendes Bild: Waren im ersten Jahr noch $26 \%$ der Patienten ohne Progression, so waren es im zweiten nur noch $11 \%$. Ähnliches findet sich in der Studie von Ducreux et al. (2000): Eine Progression war in dieser Studie bei $41 \%$ der Patienten nachweisbar. Von diesen $41 \%$ waren lediglich fünf Patienten nach schon dreimonatiger Therapie progredient. Die weit größere Zahl von 11 Patienten jedoch zeigte ihre Progredienz erst nach 6 Monaten Therapiedauer. Ein Grund hierfür könnte die sich entwickelnde Tachyphylaxie sein (siehe auch Kapitel 1.3.2):

Im Vergleich der verschiedenen Studien fällt auf, dass der Anteil der Patienten, bei denen eine stable disease beobachtet werden konnte, in den jeweiligen Studien, die einen Zeitraum von 612 Monaten beobachteten, umso kleiner wird, je länger der Beobachtungszeitraum ist. Eine Tachyphylaxie tritt unter einer Therapie mit Octreotid oder Lanreotid nach einer durchschnitt- 
lichen Behandlungsdauer von 6-18 Monaten auf. Den größten Anteil an nachgewiesener stable disease konnte die Studie von Rinke et al. (2009) deren Beobachtungszeitraum auf sechs Monate beschränkt war, aufweisen. Innerhalb dieser ersten sechs Monate der Therapie ist die Ausbildung der Tachyphylaxie selten bis gar nicht vorhanden, was den Anteil der progredient verlaufenden Tumorerkrankungen wesentlich herabsetzt. Der Anteil dieser Patienten mag hingegen in den Studien von Ducreux et al. (2000) und von Arnold et al. (1996) bereits wesentlich an Bedeutung zunehmen, da die Wahrscheinlichkeit für das Auftreten eine Tachyphylaxie in deren Beobachtungszeitraum von jeweils bis zu einem Jahr zunimmt. Weiterhin fraglich ist jedoch, welche Rolle die Ausbildung einer Tachyphylaxie bei den Patienten spielt, deren Erkrankung auch nach 18 Monaten nicht progredient ist. Die Zahlen unserer Analyse würden den Schluss nahelegen, dass diese Patienten bei andauernder Therapie auch im weiteren Verlauf keine Tachyphylaxie mehr ausbilden. Dies würde wiederum den größeren Anteil der Patienten in der stable disease unserer Beobachtung verglichen mit der Studie von Arnold et al. (1996) erklären. Hierbei ist jedoch auch zu bedenken, dass sämtliche Patienten des Kollektivs der Studie von Arnold et al. (1996) an einem metastasierten NET litten, wohingegen im Kollektiv unserer Analyse $32 \%$ der Patenten noch keine Metastasen aufwiesen. Oben genannte Annahme muss also eine Vermutung unsererseits bleiben. Weitere Studien bezüglich der Wahrscheinlichkeit einer Tachyphylaxie über eine Therapiedauer von 18 Monaten hinaus wären zur Beurteilung dieser Fragestellung erforderlich, wobei hier das Studiendesign analog $\mathrm{zu}$ bereits bestehenden Studien gewählt werden sollte, um einen exakten Vergleich ziehen zu können $\mathrm{Zu}$ beachten ist nämlich außerdem, in wie weit verschiedene Tumorarten, Differenzierungsgrade und der Rezeptorstatus zur Ausbildung einer Tachyphylaxie beitragen. In den Studien von Kaal et al. (2000) und Wahid et al. (2002) wurde eine Antikörperbildung gegen Somatostatin-Rezeptoren unter Octreotid beobachtet. Für diesen Pathomechanismus ließe sich ein Zusammenhang zwischen Rezeptorstatus und Risiko einer Tachyphylaxie gut vorstellen, was als prognostischer Marker für den Erfolg einer Langzeittherapie mit SAA gewertet werden könnte. Einen weiteren denkbaren Pathomechanismus der Tachyphylaxie stellt die Ausbildung neuer Signalwege oder die Änderung bereits bestehender Signalwege innerhalb der Tumorzellen durch Mutationen in den Rezeptoren, wie sie in Kapitel 4.3.8 beschrieben werden, dar. Auch auf diesen Gebieten sind jedoch weitere Studien erforderlich, um eine fundierte Aussage zu den Zusammenhängen machen zu können.

Die nun mehrfach beschriebene und diskutierte unterschiedliche Datenlage bezüglich des Anteils der Patienten mit stable disease und deren Dauer in den jeweiligen Studien wirft die Frage auf, in wie weit eine stable disease innerhalb eines kurzen Zeitraums überhaupt als Para- 
meter für den Erfolg einer Therapie mit SAA verwendet werden darf. Als Erkrankung in der stable disease definiert sind Tumoren, deren Größe im Beobachtungszeitraum weder um mehr als $20 \%$ zunimmt, noch um mehr als $30 \%$ abnimmt. Einige Tumoren haben jedoch per se einen spontan langsamen Wachstumsverlauf, der zu keiner Größenzunahme von mehr al $20 \%$ im beobachteten Zeitraum führt. Zu diesen Tumoren zählen vor allem gut-differenzierte NET, wie sie unter der Gesamtgruppe der NET häufig zu finden sind. Per Definitionem weißen diese Tumoren also schon von sich aus einen Verlauf in der stable disease auf. Hier gilt es, in der Betrachtung und Interpretation der Ergebnisse besonders vorsichtig vorzugehen: Diese „spontanen stable disease-Verläufe" dürfen nicht mit einer stable disease als Erfolg einer Therapie mit SAA verwechselt und somit fehlinterpretiert werden (Arnold et al. 2000).

Einen letzten zu betrachtenden Aspekt bezüglich des Verlaufs der Tumorgröße stellt das Vorkommen einer Tumorregression unter SAA dar. In der Studie von Rinke et al. (2009) konnte eine Regression bei einem der beobachteten Patienten nachgewiesen werden. Unter den Patienten unseres Kollektivs trat keine Regression ein. Lediglich bei einem Patienten konnte eine partial response beobachtet werden. Auch die Studien von Arnold et al. (1996) und von Ducreux et al. (2000) verzeichneten keine Regression.

Aufgrund der Datenlage unserer Analyse kann zusammenfassend festgestellt werden, dass SAA das Wachstum eines NET verlangsamen können, für die zytoreduktive Therapie dieser Tumoren jedoch keine Alternative darstellen.

\subsubsection{Entstehung hepatischer und sonstiger peripherer Metastasen}

Die drei Studien, die der Diskussion um die Dauer der stable disease hauptsächlich zugrunde liegen (Arnold et al. 1996; Ducreux et al. 2000; Rinke et al. 2009), differenzieren bezüglich der stable disease und der progressive disease nicht zwischen dem Verlauf des Primärtumors und dem Verlauf der Metastasierung. Als Besonderheit unserer Analyse wurde für den Begriff der „stable disease“ noch einmal gesondert zwischen dem Verlauf der Tumorgröße und dem Verlauf der Metastasen unterschieden. Auf die beobachteten Unterschiede dieser beiden Verläufe soll in den folgenden beiden Kapiteln eingegangen werden.

Beim Betrachten der prozentualen Anteile der stable disease und der progressive disease bezüglich Tumorgröße, hepatischer Metastasen und sonstiger peripherer Metastasen in unserem Patientenkollektiv ergibt sich eine jeweils weit höhere Prozentzahl der stable disease und eine weit geringere Prozentzahl an progressive disease unter SAA verglichen mit dem Zeitraum, in dem die Patienten keine Therapie mit SAA erhielten: Traten im Zeitraum ohne Therapie mit SAA sowohl am Primärtumor, als auch unter den hepatischen Metastasen noch Rezidive auf, so konnten diese unter SAA nicht mehr nachgewiesen werden (zur Definition des Rezidivs 
siehe Kapitel 2.3.2). Was das Wachstum des Primärtumors und das Ausmaß der systemischen Ausbreitung der Erkrankung betrifft, ergibt sich somit in diesen Fällen eine Übereinstimmung der Wirksamkeit von SAA.

\subsubsection{Größenverlauf der hepatischen und sonstigen peripheren Metastasen}

Unsere Ergebnisse zeigen eine Übereinstimmung des Größenverlaufs der Metastasen mit dem Größenverlauf der Primärtumoren: Verglichen mit dem Zeitraum, in dem die Patienten unserer Analyse keine SAA erhielten, kann jeweils ein größerer Anteil an Patienten mit stable disease unter SAA beobachtet werden. Der Anteil der Patienten, die unter SAA eine progressive disease aufwiesen ist im Vergleich mit dem Zeitraum ohne Therapie mit SAA dabei jeweils geringer. Außerdem kam es unter der Therapie mit SAA zu keinem Rezidiv, was im Zeitraum ohne SAA bei drei Patienten beobachtet werden konnte. Ähnliche Beobachtungen finden sich in folgenden Studien und Case-Reports wieder:

Clements und Elias (1985) veröffentlichten einen Case-Report über einen VIPom-Patienten, bei dem nach fünfmonatiger Therapie mit SAA mittels CT-Kontrolle eine Abnahme der Anzahl, Größe und Kontrastmittelaufnahme der hepatischen Metastasen beobachtet werden konnte. Ebenso erschien im gleichen Jahr ein Case-Report, in dem Shepherd und Senator (1986) bei einem Patienten, der unter einem Gastrinom litt, nach sechs Monaten Therapie mit SAA eine partielle Größenreduktion von Lebermetastasen beobachten konnten. Eine weitere interessante Beobachtung machten Eriksson et al. (1997): Sie beschrieben eine Zunahme der Apoptosen in Lebermetastasen von NET unter einer Therapie mit SAA. In dieser Studie wurde zu Beginn der Therapie und jeweils nach sechs und zwölf Monaten eine Biopsie der Lebermetastasen durchgeführt. Auffällig war eine Zunahme der Anzahl der Apoptosen mit der Dauer der Therapie, die nach zwölf Monaten ihr Maximum erreichte. Hieraus ließe sich auf den ersten Blick vermuten, dass eine Therapie mit SAA mit zunehmender Dauer auch an Effektivität gewinnt. Dies muss jedoch, wie schon zuvor angemerkt (vergleiche Kapitel 4.3.6), kontrovers diskutiert werden (Aparicio et al. 2001). Die Studie von Eriksson et al. (1997) macht keine Angaben bezüglich der Entwicklung der Apoptosen über den Zeitraum des ersten Therapiejahres hinaus. Unklar bleibt also, wie viele der Patienten nach diesem ersten Jahr durch die auftretende Tachyphylaxie doch noch einen progredienten Krankheitsverlauf aufweisen würden, womit die Anzahl der Apoptosen in einer weiteren Biopsie möglicherweise wieder geringer ausfallen würde.

Schaut man sich die Verhältnisse von stable disease und Progression im Patientenkollektiv unserer Analyse an, so fällt bei der Betrachtung unserer Ergebnisse auf, dass Zunahme an stable disease beziehungsweise Abnahme an progressive disease bezüglich der Größenverläu- 
fe der Metastasen jeweils nicht so stark ausfallen, wie sie es im Größenverlauf der Primärtumoren tun. Aus dieser Beobachtung könnte geschlossen werden, dass eine Therapie mit SAA einen größeren Effekt auf den Primärtumor als auf die entstandenen Metastasen hat. Zur Unterstützung dieser These sei das Beispiel der Gastrointestinalen Stromatumoren (GIST) genannt: In diesen Tumoren können Mutationen im Gen einer Tyrosin-Kinase nachgewiesen werden. Es besteht eine Korrelation zwischen dem Genotyp der Tyrosin-Kinase und dem Ansprechen auf eine Therapie mit Imatinib, einem Tyrosin-Kinase-Hemmer (Heinrich et al. 2008). Emile et al. (2012) wiesen verschiedene Mutationen in den Metastasen verglichen mit den Primärtumoren nach. Demnach könnte das Ansprechen auf eine Therapie mit Imatinib in Primärtumor und Metastase unterschiedlich erfolgreich sein. Ein ähnlicher Mechanismus könnte auch bei NETs vorliegen, was zur Folge hätte, dass SAA je nach Mutation in den Rezeptoren unterschiedliche Effekte auf den Größenverlauf der Metastasen beziehungsweise Primärtumoren haben. Hierzu sind jedoch weitere Studien vonnöten.

\subsubsection{Wirksamkeit der SAA im Vergleich zum Zeitraum ohne SAA in detaillierter Gruppenaufteilung}

Unter Berücksichtigung der Einteilung in die verschiedenen Untergruppen, wie sie in Kapitel 2.4 beschrieben sind, soll nun ein besonderes Augenmerk auf die Effekte von SAA im Vergleich zum Zeitraum ohne SAA, je nachdem, ob eine alternative Therapie stattfand oder nicht, gerichtet werden. Dabei betrachtet werden Verlauf der Tumormarker und der Tumorgröße sowie das Entstehen hepatischer und sonstiger peripherer Metastasen und deren Größenverlauf.

Grundsätzlich anzumerken ist dabei jedoch, dass es sich durch die Einteilung in Subgruppen in den einzelnen Stichproben jeweils um ein recht kleines Patientenkollektiv handelt. Dies hat zur Folge, dass hinsichtlich der Prüfung der Vergleiche auf Signifikanz eine so genannte „Unterpowerung“ der Tests auftreten kann, was wiederum dazu führt, dass ein Vergleich keine signifikanten Ergebnisse liefert, obwohl zu erkennende Tendenzen in einem größeren Patientenkollektiv eventuell doch einen signifikanten Unterschied ergeben würden. Hinsichtlich der Aussagekraft der einzelnen Vergleiche sei daher immer darauf hingewiesen, dass es sich bei den im Folgenden geschilderten Beobachtungen lediglich um Tendenzen handelt. Zukünftige Studien mit erheblich größeren Stichproben müssten die Signifikanz der Unterschiede zwischen den Gruppen eventuell beweisen.

Des Weiteren sei darauf hingewiesen, dass die Verläufe der oben genannten Parameter immer auch in Zusammenschau mit dem individuellen Fortschreiten der Erkrankung zu betrachten sind. Durch die Einteilung der Erkrankung mittels Grading, kann im Rahmen der Erstdiagno- 
se eine erste Einschätzung über die Prognose gemacht werden (Yao et al. 2008). Je schlechter die zu erwartende Prognose, also je höher das Grading, desto aggressiver wird die Therapie geplant (Öberg 2010). Der Anteil der Patienten mit einer prognostisch schlechteren Erkrankung ist also in der Patientengruppe derer, die während der Therapie mit SAA eine Zusatztherapie beziehungsweise nach Absetzten der SAA eine alternative Therapie erhalten haben, größer. Hinzu kommt, dass eine Chemotherapie, wie in Kapitel 1.3.5 bereits erwähnt, einen umso höheren Effekt verspricht, je höher das Grading des Tumors ist. Auch dies führt dazu, dass sich in der Gruppe der Patienten mit SAA plus Zusatztherapie beziehungsweise alternativer Therapie im Zeitraum ohne SAA mehr Patienten mit einem G3-Tumor befinden, als in den jeweils anderen Kollektiven. Beim Betrachten der individuellen Verläufe muss also auch immer der durch den Differenzierungsgrad gewissermaßen vorgegebene spontane Verlauf einer solchen Erkrankung berücksichtigt werden (siehe dazu auch Kapitel 4.3.6).

\subsubsection{Verlauf der Tumormarker}

Auffällig im Vergleich der oben definierten Gruppen ist hier, dass der Anteil der Patienten, bei denen die Konzentration der Tumormarker stabil blieb, jeweils in der Gruppe der Patienten mit alternativer Therapie am höchsten ist. Die Steigerung des Anteils an stabilen Tumormarker-Konzentrationen betrifft im Gegensatz zu einer Therapie mit SAA jeweils etwa $30 \%$ (30\% der Patienten unter SAA-Monotherapie vs. $47 \%$ der Patienten unter alternativer Therapie beziehungsweise $31 \%$ der Patienten unter SAA plus Zusatztherapie vs. $55 \%$ der Patienten unter alternativer Therapie). Betrachtet man das gesamte Patientenkollektiv aller 66 Patienten, ist also in den jeweiligen Subgruppen der Zeitraum der Therapie mit SAA dem Zeitraum ohne SAA unterlegen. Hieraus ließe sich eine Tendenz ablesen, dass Chemotherapie, Interferon- $\alpha$ oder nuklearmedizinische Therapie zur Herbeiführung eines kontrollierten Verlaufs der Tumormarker am ehesten geeignet sind. Das Design unserer Analyse lässt eine Differenzierung in die einzelnen Therapiemodalitäten, die hier unter ,,alternative Therapie“ fallen, leider nicht zu. Dies muss Gegenstand zukünftiger Studien bleiben.

Auch ist aus den durchgeführten Vergleichen ersichtlich, dass - wie zu erwarten - ein komplettes Ansprechen der Tumormarker bei keinem der Patienten der jeweiligen Gruppen eingetreten ist, wenn diese Patienten im Zeitraum ohne SAA ohne jegliche Therapie blieben. Auch im Hinblick auf eine zu erzielende Senkung der Konzentrationen in den jeweiligen Normbereich wurde hier die Beobachtung gemacht, dass sich diese im Zeitraum ohne SAA nur durch die Durchführung einer alternativen Therapie erreichen lässt. Für das Auftreten eines kompletten Ansprechens insgesamt scheint eine Therapie mit SAA, wie in Kapitel 4.3.4 beschrieben, den alternativen Therapien überlegen zu sein. Dies würde bei weiterer Unterteilung in die 
Subgruppen aber nur für eine Durchführung von SAA plus Zusatztherapie gelten. Die Gabe von SAA als Monotherapie hingegen scheint in diesem Punkt der alternativen Therapie unterlegen. (Zur Definition der alternativen Therapien siehe Kapitel 4.2)

\subsubsection{Verlauf der Tumorgröße}

Vergleicht man die genannten Gruppen hinsichtlich des Verlaufs der Tumorgröße, so ist aus unseren Ergebnissen ersichtlich, dass die jeweils größte Anzahl an Verläufen in der stable disease während der Therapie mit SAA verzeichnet werden konnten. Dabei scheint es, bei Betrachtung der prozentualen Anteile der entsprechenden Gruppen, einen Unterschied zu machen, ob SAA als Monotherapie oder mit einer Zusatztherapie verabreicht werden: In der Gruppe der Patienten mit Zusatztherapie lag die Anzahl der Patienten, die eine stable disease erlangten, um 9 \%-Punkte höher als in der Gruppe mit SAA-Monotherapie (43\% der Patienten unter SAA-Monotherapie vs. $52 \%$ der Patienten unter SAA plus Zusatztherapie). Diese Annahme kann von der bereits erwähnten Studie von Öberg et al. (2004) unterstützt werden. Dort konnte ein höherer antiproliferativer Effekt unter SAA kombiniert mit Interferon- $\alpha$ nachgewiesen werden (Vergleiche Kapitel 1.3.3). Ob der größere Anteil an stable disease unter SAA plus Zusatztherapie jedoch alleine auf einen Kombination mit Interferon- $\alpha$, oder auch auf andere zusätzliche Therapien zurückzuführen ist, kann den Daten dieser Analyse nicht entnommen werden und muss Gegenstand zukünftiger Studien bleiben. Des Weiteren zeigt sich jeweils eine niedrigere Anzahl an progressive disease unter SAA im Vergleich zum Zeitraum ohne SAA. Wie in Kapitel 4.3.6 beschrieben, zeigte sich einer der Patienten unter SAA in der partial response, welche im Zeitraum ohne SAA bei keinem der Patienten auftrat. Wie bereits ebenfalls in Kapitel 4.3.6 beschrieben, zeigte unter SAA keiner der Patienten ein Rezidiv auf, wohingegen das Auftreten von Rezidiven im Zeitraum ohne SAA beobachtet werden konnte.

Hinsichtlich der Unterschiede der Therapieregime im Zeitraum ohne SAA scheinen die Patienten mit alternativen Therapien bezüglich des Auftretens einer stable disease beziehungsweise der Rezidive jeweils den ungünstigsten Verlauf zu haben: Der Anteil der stable disease in den entsprechenden Gruppen ist jeweils am geringsten, wobei die Anzahl der Rezidive jeweils am höchsten ist. Hier sei aber noch einmal darauf hingewiesen, dass Patienten, die eine alternative, meist in ein aggressives Regime eingebettete Therapie erhalten, einen per se ungünstigeren Verlauf der Erkrankung zu befürchten haben. Schaut man sich die absoluten Zahlen der jeweiligen Untergruppen bezüglich der progressive disease an, so ist die Durchführung einer alternativen Therapie dennoch gerechtfertigt, da unter ihr weniger Progressionen zu verzeich- 
nen waren als in den Gruppen ohne jegliche Therapie. (Zur Definition der unterschiedlichen Patientengruppen siehe Kapitel 2.4)

\subsubsection{Entstehung hepatischer und sonstiger peripherer Metastasen}

Eine Unterteilung in die genannten Untergruppen verändert die in Kapitel 4.3.7 beschriebenen Beobachtungen nicht: Insgesamt ist der Anteil der stabilen Krankheitsverläufe hinsichtlich der Metastasierung jeweils unter SAA höher als im Zeitraum ohne SAA. Dabei besteht kein wesentlicher Unterschied zwischen einer SAA-Monotherapie und SAA plus Zusatztherapie was die hepatischen Metastasen betrifft. Für die Entstehung sonstiger peripherer Metastasen ist zu erwähnen, dass die Anzahl der neuen Metastasen in der Gruppe der Patienten, die SAA plus Zusatztherapie erhielten, höher ist als in der Gruppe unter SAA-Monotherapie: Unter SAAMonotherapie sind dies lediglich $16 \%$, wohingegen in der Gruppe der SAA plus Zusatztherapie $35 \%$ der Patienten neue Metastasen entwickelten. Denkbar wäre auch hier, dass es sich bei den Tumoren, die mit SAA plus Zusatztherapie behandelt wurden, um Tumoren mit einem niedrigen Differenzierungsgrad handelt. Diese Tumoren sprechen, wie in Kapitel 1.3.5 erwähnt, eher schlecht auf eine Therapie mit SAA an. Ein vermehrtes Auftreten neuer Metastasen wäre somit Ausdruck dieser aggressiv verlaufenden Erkrankungen. Für den Zeitraum ohne SAA unterscheiden sich die Therapieregime bezüglich des Neuauftretens von Metastasen insgesamt nicht voneinander.

\subsubsection{Größenverlauf der hepatischen und sonstigen peripheren Metastasen}

Insgesamt gelten auch hier die in Kapitel 4.3.9 diskutierten Beobachtungen. Bei der dort stattgefundenen Betrachtung des gesamten Patientenkollektivs stellt sich ein jeweils günstigerer Verlauf der Erkrankung unter SAA dar. Dabei verhalten sich hepatische und periphere Metastasen in etwa gleich. Die nun erfolgte Aufschlüsselung in die Untergruppen ergibt jedoch einen Unterschied in den Größenverläufen der hepatischen verglichen mit den sonstigen peripheren Metastasen: Für den Vergleich „SAA-Monotherapie“ vs. „SAA plus Zusatztherapie“ scheint bezüglich der hepatischen Metastasen kein wesentlicher Unterschied zu bestehen. Bezüglich der sonstigen peripheren Metastasen jedoch lässt sich eine Unterlegenheit der SAA plus Zusatztherapie im Vergleich zu den anderen Regimen vermuten: Während im Patientenkollektiv der SAA-Monotherapie die Anzahl an Verläufen in der stable disease am höchsten und die Anzahl der progressive disease am niedrigsten ist, ergibt sich ein Abfall der Erkrankungen in der stable disease und eine Zunahme der Progressionen unter SAA plus Zusatztherapie verglichen mit den jeweiligen Regimen des Zeitraums ohne SAA untereinander. Dies mag daran liegen, dass SAA plus Zusatztherapie vor allem bei prognostisch ungünstigen Tumoren eingesetzt werden. Wie auch in Kapitel 1.3.5 erwähnt sind NETs mit einem geringen 
Differenzierungsgrad in den meisten Fällen einer Chemotherapie besser zugänglich als einer Therapie mit SAA. Darin mag die Überlegenheit der alternativen Therapien gegenüber SAA plus Zusatztherapie hinsichtlich der stable disease in unsere Analyse begründet sein (vergleiche Abbildung 36). 


\section{Zusammenfassung}

In der vorliegenden retrospektiven Analyse wurden die Daten von 66 Patienten, die an einem NET erkrankt und in der Universitätsmedizin Göttingen behandelt wurden, bezüglich der Effekte der Therapie mit SAA statistisch aufgearbeitet, ausgewertet und die erhaltenen Daten anhand der aktuellen Literatur diskutiert.

Ziel war es, die in vorherigen Studien beobachtete verlängerte Dauer der stable disease unter der Therapie mit SAA auch in unserem Patientenkollektiv nachzuweisen. Ein zusätzliches Augenmerk lag auf weiteren Effekten der Therapie mit SAA: Verlauf der Tumormarker und der Symptomatik sowie Verlauf der Tumorgröße und der Metastasen, auch im Vergleich von SAA-Monotherapie vs. SAA plus Zusatztherapie. Dazu wurden die Daten der Patienten bezüglich oben genannter Aspekte ausgewertet und mit der Therapie in Beziehung gesetzt. Neben anamnestischen Angaben und Laborwerten dienten hierfür auch Daten aus der Bildgebung mittels CT, MRT und Ultraschall. Für die Aspekte der Symptomkontrolle, der Kontrolle der Tumormarker sowie des Tumorwachstums und der Entstehung von Metastasen konnte eine Überlegenheit des Zeitraums mit SAA gegenüber dem Zeitraum ohne SAA nachgewiesen werden. Der lange Beobachtungszeitraum und die dadurch nicht unerhebliche Anzahl von Patienten, die während der Analyse ausschieden, sowie die mit den Jahren veränderte und verbesserte Diagnostik (vor allem im Bereich der Tumormarker) und das Fehlen standardisierter Zeitintervalle bezüglich der Verlaufskontrolle sind jedoch als Einschränkung dieser Analyse zu nennen.

Im beobachteten Zeitraum vom 1. Januar 1975 bis 31. August 2011 ergab sich eine im Vergleich zu vorherigen Studien recht lange mittlere Beobachtungsdauer von 102 Monaten, in denen im Schnitt eine Monotherapie mit SAA über 28 Tage und eine Gesamtdauer der Therapie mit SAA über 47 Monate nachgewiesen werden konnte. Dieser von uns gezeigte und im Vergleich zu anderen, oben genannten Studien sehr lange Beobachtungszeitraum und das damit verbundene gute Langzeitüberleben lassen auf eine Proliferationshemmung und somit auf eine lange Dauer der stable disease schließen. Bezüglich der Symptomatik, die historisch gesehen die wichtigste Indikation zu einer solchen Therapie darstellt, konnte in unserem Kollektiv bei $17,8 \%$ der Patienten $(n=8)$ eine Verbesserung unter SAA beobachtet werden. Die Werte der Tumormarker blieben unter einer Therapie mit SAA in 30,3\% der Fälle $(n=20)$ stabil. Die durchschnittliche Dauer der stable disease unter SAA betrug in unserer Analyse 21 Monate. Des Weiteren zeigt sich in unserem Kollektiv kein Unterschied in der Dauer der stable disease bezüglich des initialen Metastasierungsstadiums oder der Anwendung von zusätzlichen Therapien. Im Größenverlauf des Primärtumors konnte bei $47 \%$ der Patienten $(n=31)$ 
eine stable disease beobachtet werden. Wie schon in vorausgegangenen Studien beschrieben, bestätigen auch unsere Daten, dass zwischen der Dauer der stable disease und dem Verlauf der Tumormarker und der Symptomatik keine Korrelation besteht. Es zeigte sich eine Übereinstimmung von Wachstum des Primärtumors und Ausmaß der systemischen Ausbreitung, wobei Daten unserer Analyse zeigen, dass die Therapie mit SAA einen stärkeren Effekt auf den Verlauf des Primärtumors als auf den Verlauf bereits bestehender Metastasen hat.

Anhand der in unserer Auswertung erzielten Ergebnisse kann gezeigt werden, dass die Beobachtungen in unserem Patientenkollektiv mit denen der aktuellen Literatur vergleichbar sind. Festzuhalten bleibt somit, dass eine Therapie mit SAA den Krankheitsverlauf eines NET im Sinne einer stable disease um bis zu 21 Monate verzögern kann. Im besten Falle führt eine solche Therapie innerhalb dieses Zeitraums zusätzlich zu einer Besserung der klinischen Symptomatik. Eine Größenregredienz sowohl des Primärtumors als auch der Metastasen, wie sie auch nur vereinzelt in den früheren Studien beschrieben worden waren, konnte jedoch in unserem Patientenkollektiv nicht beobachtet werden. Dies unterstreicht die Aussage, dass es sich bei SAA um ein antiproliferatives, aber nicht zytoreduktives Therapiekonzept handelt. Studien, die neue Substanzen zur Behandlung von NETs zum Teil mit bereits großem Erfolg getestet haben, verdeutlichen die Suche nach einer immer effektiveren und individuelleren Therapie dieser Erkrankung. Langzeitergebnisse diesbezüglich bleiben jedoch abzuwarten. 


\section{Literaturverzeichnis}

Aparicio T, Ducreux M, Baudin E, Sabourin JC, De Baere T, Mitry E, Schlumberger M, Rougier P (2001): Antitumor activity of somatostatin analogues in progressive metastatic neuroendocrine tumors. Eur J Cancer 37(8), 1014-1019

Arnold R, Trautmann ME, Creutzfeldt W, Benning R, Benning M, Neuhaus C, Jurgensen R, Stein K, Schafer H, Bruns C, Denner HJ (1996): Somatostatin analogue octreotide and inhibition of tumour growth in metastatic endocrine gastroenteropancreatic tumours. Gut $\underline{38}, 430-438$

Arnold R, Simon B, Wied M (2000): Treatment of Neuroendocrine GEP Tumours with Somatostatin Analogues, A Review. Digestion 62(1), 84-91

Arnold R, Wied M, Behr TH (2002): Somatostatin analogues in the treatment of endocrine tumors of the gastrointestinal tract. Expert Opin Pharmacother $\underline{3}(6), 643-656$

Bajetta E, Ferrari L, Martinetti A, Celio L, Procopio G, Artale S, Zilembo N, Di Bartolomeo M, Seregni E, Bombardieri E (1999): Chromogranin A, neuron specific enolase, carcinoembryonic antigen, and hydroxyindole acetic acid evaluation in patients with neuroendocrine tumors. Cancer $\underline{86}(5), 858-865$

Bajetta E, Procopio G, Catena L, Martinetti A, De Dosso S, Ricci S, Lecchi AS, Boscani PF, Iacobelli S, Carteni G (2006): Lanreotide Autogel Every 6 Weeks Compared With Lanreotide Microparticles Every 3 Weeks in Patients With Well Differentiated Neuroendocrine Tumors: A Phase III Study. Cancer 107(10), 2474-2481

Bajetta E, Procopio G, Pusceddu S, Pietrantonio P, Milione M, Maccauro M, Verzoni E, Guadalupi V, Platania M (2009): From biology to clinical experience: evolution in the knowledge of neuroendocrine tumours. Oncology Reviews $\underline{3}(2), 79-87$

Bauer H, Schmidt GF (1980): Experimental and clinical studies on the effect of combined treatment with cimetidine and somatostatin. Z Gastroenterol 18(6), 314-319

Beaumont V, Hepworth MB, Luty JS, Kelly E, Henderson G (1998): Somatostatin receptor desensitization in NG108-15 cells. A consequence of receptor sequestration. J Biol Chem 273(50), 33174-33183

Burke AP, Thomas $\quad$ RM, Elsayed AM, Sobin $\quad$ LH $\quad$ (1997): Carcinoids of the jejunum and ileum: an immunohistochemical and clinicopathologic study of 167 cases. Cancer, $\underline{79}$ (6), 1086-1093

Calender A (2000): Molecular genetics of neuroendocrine tumours. Digestion 62 (1), 3-18

Cameron S, Ramadori G (2010): Der Beitrag der Gastroenterologie zur Therapie - 15 Jahre Erfahrung: aus der Vielfalt Hoffnung schöpfen. GlandulaNeT 13(10), 49-54 
Caplin ME, Pavel M, Ćwikła JB, Phan AT, Raderer M, Sedláčková E, Cadiot G, Wolin EM, Capdevila J, Wall L; CLARINET Investigators (2014): Lanreotide in metastatic enteropancreatic neuroendocrine tumors. N Engl J Med 371(3), 224233

Cattoretti G, Becker MH, Key G, Duchrow M, Schlüter C, Galle J, Gerdes J (1992): Monoclonal antibodies against recombinant parts of the Ki-67 antigen (MIB 1 and MIB 3) detect proliferating cells in microwave-processed formalin-fixed paraffin sections. $\mathbf{J}$ Pathol $\underline{168}(4), 357-363$

Challacombe DN, Brown GA, Black SC, Storrie MH (1972): Increased excretion of 5hydroxyindoleacetic acid in urine of children with untreated coeliac disease. Arch Dis Child $\underline{47}(235), 442-445$

Clements D, Elias E (1985): Regression of metastatic vipoma with somatostatin analogue SMS. Lancet 1 (8433), 874-875

Creutzfeldt W (1996): Carcinoid Tumors: Development of Our Knowledge. World J Surg $\underline{20}(2), 126-131$

Creutzfeldt W, Bartsch HH, Jacubaschke U, Stöckmann F (1991): Treatment of gastrointestinal endocrine tumours with interferon-alpha and octreotide. Acta Oncol 30(4), 529535

Csaba Z, Dournaud P (2001): Cellular biology of Somatostatin receptors. Neuropeptides $\underline{35}(1), 1-23$

Degorce F, Goumon Y, Jacquemart L, Vidaud C, Bellager L, Pons Anicet D, Seguin P, MetzBoutigue MH, Aunis D (1999): A new human chromogranin A (CgA) immunoradiometric assay involving monoclonal antibodies raised against the unprocessed central domain. Br J Cancer 79(1), 65-71

Desai DC, O'Dorisio TM, Schirmer WJ, Jung SS, Khabiri H, Villanueva V, Martin EW (2001): Serum pancreastatin levels predict response to hepatic artery chemoembolization and somatostatin analogue therapy in metastatic neuroendocrine tumors. Regul Pept $\underline{96}(3), 113-117$

Dierdorf SF (2003): Carcinoid tumor and carcinoid syndrome. Curr Opin Anaesthesiol 16(3), $343-347$

Ducreux M, Ruszniewski P, Chayvialle JA, Blumberg J, Cloarec D, Michel H, Raymond JM, Dupas JL, Gouerou H, Jian R (2000): The antitumoral effect of the long-acting somatostatin analog lanreotide in neuroendocrine tumors. Am J Gastroenterol 95(11), 32763281 
Dueno MI, Bai JC, Santangelo WC, Krejs GJ (1987): Effect of somatostatin analog on water and electrolyte transport and transit time in human small bowel. Dig Dis Sci $\underline{32}(10)$, 1092-1096

Duerr EM, Mizukami Y, Ng, A, Xavier RJ, Kikuchi H, Deshpande V, Warshaw AL, Glickman J, Kulke MH, Chung DC (2008): Defining molecular classifications and targets in gastroenteropancreatic neuroendocrine tumors through DNA microarray analysis. Endocr Relat Cancer 15(1), 243-256

Eisenhauer EA, Therasse P, Bogaerts J, Schwartz LH, Sargent D, Ford R, Dancey J, Arbuck S, Gwyther S, Mooney M (2009): New response evaluation criteria in solid tumours: revised RECIST guideline (version 1.1). Eur J Cancer 45(2), 228-247

Emile JF, Brahimi S, Coindre JM, Bringuier PP, Monges G, Samb P, Doucet L, Hostein I, Landi B, Buisine MP (2012): Frequencies of KIT and PDGFRA mutations in the MolecGIST prospective population-based study differ from those of advanced GISTs. Med Oncol 29(3), 1765-1772

Eriksson B, Renstrup J, Imam H, Öberg K (1997): High-dose treatment with lanreotide of patients with advanced neuroendocrine gastrointestinal tumors: clinical and biological effects. Ann Oncol $\underline{8}(10), 1041-1044$

Eriksson B, Klöppel G, Krenning E, Ahlman H, Plöckinger U, Wiedenmann B, Arnold R, Auernhammer C, Körner M, Rindi G (2008): Consensus guidelines for the management of patients with digestive neuroendocrine tumors-well-differentiated jejunal-ileal tumor/carcinoma. Neuroendocrinology $\underline{87}(1), 8-19$

Escorsell A, Bandi JC, Andreu V, Moitinho E, García-Pegàn JC, Bosch J, Rodés J (2001): Desensitization to the effects of intravenous octreotide in cirrhotic patients with portal hypertension. Gastroenterology 120(1), 161-169

European Neuroendocrine Tumor Society (2012): Abstracts of the $9^{\text {th }}$ Annual ETNETS Conference for the Diagnosis and Treatment of Neuroendocrine Tumor Diseases. Neuroendocrinology $\underline{96}(1), 1-72$

Faiss S, Räth U, Mansmann U, Caird D, Clemens N, Riecken EO, Wiedenmann B (1999): Ultra-high-dose lanreotide treatment in patients with metastatic neuroendocrine gastroenteropancreatic tumors. Digestion $\underline{60}(5), 469-476$

Falconi M, Plockinger U, Kwekkeboom DJ, Manfredi R, Korner M, Kvols L, Pape UF, Ricke J, Goretzki PE, Wildi S (2006): Well-differentiated pancreatic nonfunctioning tumors/carcinoma. Neuroendocrinology $\underline{84}(3), 196-211$ 
Fan ST, Le Treut YP, Mazzaferro V, Burroughs AK, Olausson M, Breitenstein S, Frilling A (2015): Liver transplantation for neuroendocrine tumour liver metastases. HPB (Oxford) $17(1), 23-28$

Feldman JM (1986): Urinary serotonin in the diagnosis of carcinoid tumors. Clin Chem $\underline{32}(5)$, $840-844$

Fottner C, Weber MM (2012): Neuroendocrine neoplasms of the gastrointestinal tract. Classification, clinical presentation and diagnosis. Internist (Berl) $\underline{53}(2), 131-144$

Garland J, Buscombe JR, Bouvier C, Bouloux P, Chapman MH, Chow AC, Reynolds N, Caplin ME (2003): Sandostatin LAR (long-acting octreotide acetate) for malignant carcinoid syndrome: a 3-year experience. Aliment Pharmacol Ther 17(3), 437-444

Gerdes J, Li L, Schlueter C, Duchrow M, Wohlenberg C, Gerlach C, Stahmer I, Kloth S, Brandt E, Flad HD (1991): Immunobiochemical and molecular biologic characterization of the cell proliferation-associated nuclear antigen that is defined by monoclonal antibody Ki-67. Am J Pathol 138(4), 867-873

Grozinsky-Glasberg S, Shimon I, Korbonits M, Grossman AB (2008): Somatostatin analogues in the control of neuroendocrine tumours: efficacy and mechanisms. Endocr Relat Cancer 15(3), 701-720

Halfdanarson TR, Rabe KG, Rubin J, Petersen GM (2008): Pancreatic neuroendocrine tumors (PNETs): incidence, prognosis and recent trend toward improved survival. Ann Oncol $\underline{19}(10), 1727-1733$

Hauso O, Gustafsson BI, Kidd M, Waldum HL, Drozdov I, Chan AK, Modlin IM (2008): Neuroendocrine tumor epidemiology: contrasting Norway and North America. Cancer $\underline{113}(10), 2655-2664$

Heinrich MC, Owzar K, Corless CL, Hollis D, Borden EC, Fletcher CD, Ryan CW, von Mehren M, Blanke CD, Rankin C (2008): Correlation of kinase genotype and clinical outcome in the North American Intergroup Phase III Trial of imatinib mesylate for treatment of advanced gastrointestinal stromal tumor: CALGB 150105 Study by Cancer and Leukemia Group B and Southwest Oncology Group. J Clin Oncol 26(33), 5360-5367

Hipkin RW, Friedman J, Clar RB, Eppler CM, Schonbrunn A (1997): Agonist-induced desensitization, internalization and phosphorylation of the sst $2 \mathrm{~A}$ somatostatin receptor. $\mathrm{J}$ Biol Chem 272(21), 13867-13876

Hofland LJ, Lamberts SW (2003): The pathophysiological consequences of somatostatin receptor internalization and resistance. Endocr Rev 24(1), 28-47 
Hofland LJ, de Herder WW, Waaijers M, Zuijderwijk J, Uitterlinden P, van Koetsveld PM, Lamberts SW (1999): Interferon-alpha-2a is a potent inhibitor of hormone secre-

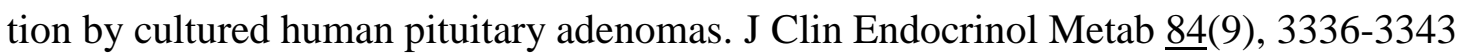

Hotz HG, Bojarski C, Buhr HJ (2011): Neuroendocrine colorectal tumors. Surgical and endoscopic treatment. Chirurg $\underline{82}(7), 607-611$

Janson ET, Öberg K (1993): Long-term management of the carcinoid syndrome. Treatment with octreotide alone and in combination with alpha-interferon. Acta Oncol $\underline{32}(2)$, 225-229

Janson ET, Holmberg L, Stridsberg M, Eriksson B, Theodorsson E, Wilander E, Öberg K (1997): Carcinoid tumors: analysis of prognostic factors and survival in 301 patients from a referral center. Ann Oncol $\underline{8}(7), 685-690$

Kaal A, Orskov H, Nielsen S, Pedroncelli AM, Lancranjan I, Marbach P, Weeke J (2000): Occurrence and effects of octreotide antibodies during nasal, subcutaneous and slow release intramuscular treatment. Eur J Endocrinol 143(3), 353-361

Kaltsas GA, Mukherjee JJ, Isidori A, Kola B, Plowman PN, Monson JP, Grossman AB, Besser GM (2002): Treatment of advanced neuroendocrine tumours using combination chemotherapy with lomustine and 5-fluorouracil. Clin Endocrinol (Oxf) 57(2), 169-83

Kaltsas GA, Besser GM, Grossman AB (2004): The diagnosis and medical management of advanced neuroendocrine tumors. Endocr Rev 25(3), 458-511

Kema IP, Schellings AM, Meiborg G, Hoppenbrouwers CJ, Muskiet FA (1992): Influence of a serotonin- and dopamine-rich diet on platelet serotonin content and urinary excretion of biogenic amines and their metabolites. Clin Chem $\underline{38}(9), 1730-1736$

Kölby L, Persson G, Franzén S, Ahrén B (2003): Randomized clinical trial of the effect of interferon alpha on survival in patients with disseminated midgut carcinoid tumours. Br J Surg 90(6), 687-693

Korse CM, Bonfrer JM, Aaronson NK, Hart AA, Taal BG (2009): Chromogranin A as an alternative to 5-hydroxyindoleacetic acid in the evaluation of symptoms during treatment of patients with neuroendocrine tumors. Neuroendocrinology $\underline{89}$ (3), 296-301

Kulke MH, Mayer RJ (1999): Carcinoid tumors. N Engl J Med 340(11), 858-868

Kwekkeboom DJ, Krenning EP, Lebtahi R, Komminoth P, Kos-Kudła B, de Herder WW, Plöckinger U; Mallorca Consensus Conferene participants; European Neuroendocrine Tumor Society (2009): ENETS Consensus Guidelines for the Standards of Care in Neuroendocrine Tumors: peptide receptor radionuclide therapy with radiolabeled somatostatin analogs. Neuroendocrinology 90(2), 220-226 
Leja J, Essaghir A, Essand M, Wester K, Oberg K, Tötterman TH, Lloyd R, Vasmatzis G, Demoulin JB, Giandomenico V (2009): Novel markers for enterochromaffin cells and gastrointestinal neuroendocrine carcinomas. Mod Pathol 22(2), 261-272

Metz DC, Jensen RT (2008): Gastrointestinal neuroendocrine tumors: pancreatic endocrine tumors. Gastroenterology 135(5), 1469-1492

Mitry E, Rougier P (2001): The treatment of undifferentiated neuroendocrine tumors. Crit Rev Oncol Hematol 37(1), 47-51

Modlin IM, Oberg K, Chung DC, Jensen RT, de Herder WW, Thakker RV, Caplin M, Delle Fave G, Kaltsas GA, Krenning EP (2008): Gastroenteropancreatic neuroendocrine tumours. Lancet Oncol $\underline{9}(1), 61-72$

Moertel CG, Sauer WG, Dockerty MB, Baggenstoss AH (1961): Life history of the carcinoid tumor of the small intestine. Cancer $\underline{14}, 901-912$

Moller LN, Stidsen CE, Hartmann B, Holst JJ (2003): Somatostatin receptors. Biochim Biophys Acta $\underline{1616}(1), 1-84$

Öberg KE (2010): Gastrointestinal neuroendocrine tumors. Ann Oncol 21(7), 72-80

Öberg K, Castellano D (2011): Current knowledge on diagnosis and staging of neuroendocrine tumors. Cancer Metastasis Rev 30(1), 3-7

Öberg KE, Janson ET, Eriksson B (1999): Tumormarkers in neuroendocrine tumors. Ital J Gastroenterol Hepatol 31(2), 160-162. Abstact

Öberg K, Kvols L, Caplin M, Delle Fave G, de Herder W, Rindi G, Ruszniewski P, Woltering EA, Wiedenmann B (2004): Consensus report on the use of somatostatin analogs for the management of neuroendocrine tumors of the gastroenteropancreatic system. Ann Oncol 15 $\underline{15}$ (6), 966-973

Öberg K, Ferone D, Kaltsas G, Knigge UP, Taal B, Plöckinger U; Mallorca Consensus Conferene participants; European Neuroendocrine Tumor Society (2009): ENETS Consensus Guidelines for the Standards of Care in Neuroendocrine Tumors: biotherapy. Neuroendocrinology 90(2), 209-213

Oberndorfer S (1907): Karzenoide Tumoren des Dünndarms. Frankf Zschr Pathol 1, 426-430

O'Toole D, Grossman A, Gross D, Delle Fave G, Barkmanova J, O'Connor J, Pape UF, Plöckinger U; Mallorca Consensus Conferene participants; European Neuroendocrine Tumor Society (2009): ENETS Consensus Guidelines for the Standards of Care in Neuroendocrine Tumors: biochemical markers. Neuroendocrinology 90(2), 194-202 
Pagès P, Benali N, Saint-Laurent N, Estève JP, Schally AV, Tkaczuk J, Vayesse N, Susini C, Buscail L (1999): sst2 somatostatin receptor mediates cell cycle arrest and induction of p27 (Kip1). Evidence for the role of Shp-1. J Biol Chem 274(21), 15186-15193

Panzuto F, Boninsegna L, Fazio N, Campana D, Pia Brizzi M, Capurso G, Scarpa A, De Braud F, Dogliotti L, Tomassetti P (2011): Metastatic and locally advanced pancreatic endocrine carcinomas: analysis of factors associated with disease progression. J Clin Oncol 29(17), 2372-2377

Pavel M, Baudin E, Couvelard A, Krenning E, Öberg K, Steinmüller T, Anlauf M, Wiedenmann B, Salazar R; Barcelona Consensus Conference participants (2012): ENETS Consensus Guidelines for the management of patients with liver and other distant metastases from neuroendocrine neoplasms of foregut, midgut, hindgut, and unknown primary. Neuroendocrinology $\underline{95}(2), 157-176$

Pirker RA, Pont J, Pöhnl R, Schütz W, Griesmacher A, Müller MM (1998): Usefulness of chromogranin A as a marker for detection of relapses of carcinoid tumours. Clin Chem Lab Med 36 (11), 837-840

Plöckinger U, Rindi G, Arnold R, Eriksson B, Krenning EP, de Herder WW, Goede A, Caplin M, Oberg K, Reubi JC; European Neuroendocrine Tumor Society (2004): Guidelines for the diagnosis and treatment of neuroendocrine gastrointestinal tumours. A consensus statement on behalf of the European Neuroendocrine Tumour Society (ENETS). Neuroendocrinology $\underline{80}(6), 394-424$

Raymond E, Dahan L, Raoul JL, Bang YJ, Borbath I, Lombard-Bohas C, Valle J, Metrakos P, Smith D, Vinik A (2011): Sunitinib malate for the treatment of pancreatic neuroendocrine tumors. N Engl J Med 364(6), 501-513

Remes SM, Tuominen VJ, Helin H, Isola J, Arola J (2012): Grading of neuroendocrine tumors with Ki-67 requires high-quality assessment practices. Am J Surg Pathol 36(9), 1359-1363

Reubi JC (2004): Somatostatin and other Peptide receptors as tools for tumor diagnosis and treatment. Neuroendocrinology $\underline{80}(1), 51-56$

Reubi JC, Waser B, Schaer JC, Laissue JA (2001): Somatostatin receptor sst1-sst5 expression in normal and neoplastic human tissues using receptor autoradiography with subtypeselective ligands. Eur J Nucl Med $\underline{28}(7), 836-846$

Ricke J, Klose KJ, Mignon M, Oberg K, Wiedenmann B (2001): Standardisation of imaging in neuroendocrine tumours: results of a European delphi process. Eur J Radiol 37(1), 8-17 
Riemann JF, Fischbach W, Galle PR, Mössner J: Gastroenterologie - das Referenzwerk für Klinik und Praxis. Georg Thieme Verlag, Stuttgart 2010

Rindi G, Klöppel G (2004): Endocrine tumors of the gut and pancreas tumor biology and classification. Neuroendocrinology $\underline{80}(1), 12-15$

Rindi G, Klöppel G, Alhman H, Caplin M, Couvelard A, de Herder WW, Erikssson B, Falchetti A, Falconi M, Komminoth P; Frascati Consensus Conference participants; European Neuroendocrine Tumor Society (2006): TNM staging of foregut (neuro)endocrine tumors: a consensus proposal including a grading system. Virchows Arch $\underline{449}(4), 395-401$

Rindi G, Klöppel G, Couvelard A, Komminoth P, Körner $\quad$ M, Lopes JM, McNicol AM, Nilsson O, Perren A, Scarpa A (2007): TNM staging of midgut and hindgut (neuro) endocrine tumors: a consensus proposal including a grading system. Virchows Arch $\underline{451}(4), 757-762$

Rinke A, Müller HH, Schade-Brittinger C, Klose KJ, Barth P, Wied M, Mayer C, Aminossadati B, Pape UF, Bläker M; PROMID Study Group (2009): Placebo-controlled, double-blind, prospective, randomized study on the effect of octreotide LAR in the control of tumor growth in patients with metastatic neuroendocrine midgut tumors: a report from the PROMID Study Group. J Clin Oncol 27(28), 4656-4663

Ritzel U, Leonhardt U, Stöckmann F, Ramadori G (1995): Treatment of metastasized midgut carcinoids with dacarbazine. Am J Gastroenterol 90(4), 627-631. Abstract

Roche A, Girish BV, de Baère T, Baudin E, Boige V, Elias D, Lasser P, Schlumberger M, Ducreux M (2003): Trans-catheter arterial chemoembolization as first-line treatment for hepatic metastases from endocrine tumors. Eur Radiol 13(1), 136-140

Rubin J, Ajani J, Schirmer W, Venook AP, Bukowski R, Pommier R, Saltz L, Dandona P, Anthony L (1999): Octreotide acetate long-acting formulation versus open-label subcutaneous octreotide acetate in malignant carcinoid syndrome. J Clin Oncol 17(2), 600-606

Scherübl H, Jensen RT, Cadiot G, Stölzel U, Klöppel G (2010): Neuroendocrine tumors of the small bowels are on the rise: Early aspects and management. World J Gastrointest En$\operatorname{dosc} 2$ (10), 325-334

Schmid HA, Schoeffter P (2004): Functional activity of the multiligand analog SOM230 at human recombinant somatostatin receptor subtypes supports its usefulness in neuroendocrine tumors. Neuroendocrinology $\underline{80}(1), 47-50$ 
Schnirer, II, Yao JC, Ajani JA (2003): Carcinoid-a comprehensive review. Acta Oncol 42(7), $672-692$

Schwörer H, Münke H, Stöckmann F, Ramadori G (1995): Treatment of diarrhea in carcinoid syndrome with ondansetron, tropisetron, and clonidine. Am J Gastroenterol 90(4), 645-648

Scoazec JY (2013): Angiogenesis in neuroendocrine tumors: therapeutic applications. Neuroendocrinology $97(1), 45-56$

Sharma K, Srikant CB (1998): Induction of wild type p53, Bax and acidic endonuclease during somatostatin-signalled apoptosis in MCF-7 human breast cancer cells. Int J Cancer $\underline{76}(2), 259-266$

Shepherd JJ, Senator GB (1986): Regression of liver metastases in patient with gastrinsecreting tumour treated with SMS 201-995. Lancet 2(8506), 574

Sobin L, Gospodarowicz M, Wittekind C: UICC: TNM Classification of Malignant Tumors. $7^{\text {th }}$ Edition, Wiley-Blackwell, Hoboken 2009

Stalla GK: Therapieleitfaden Hypophysenerkrankungen. Uni-med-Verlag, Bremen 2006

Stolk MFJ, van Erpecum KJ, Koppeschaar HPF, de Bruin WI, Jansen JBMJ, Lamers CBHW, van Berge Henegouwen GP (1993): Postprandial gall bladder motility and hormone release during intermittent and continuous subcutaneous octreotide treatment in acromegaly. Gut $\underline{34}(6), 808-813$

Strosberg JR, Cheema A, Kvols LK (2011 a): A review of systemic and liver-directed therapies for metastatic neuroendocrine tumors of the gastroenteropancreatic tract. Cancer Control $\underline{18}(2), 127-137$

Strosberg JR, Cheema A, Weber J, Han G, Coppola D, Kvols LK (2011 b): Prognostic validity of a novel American Joint Committee on Cancer Staging Classification for pancreatic neuroendocrine tumors. J Clin Oncol 29(22), 3044-3049

Teotia M, Teotia SP (1975): Serotonin metabolism in children with kwashiorkor. Am J Clin Nutr $\underline{28}(11), 1284-1288$

Therasse P, Arbuck SG, Eisenhauer EA, Wanders J, Kaplan RS, Rubinstein L, Verweij J, Van Glabbeke M, van Oosterom AT, Christian MC (2000): New guidelines to evaluate the response to treatment in solid tumors. European Organization for Research and Treatment of Cancer, National Cancer Institute of the United States, National Cancer Institute of Canada. J Natl Cancer Inst 92(3), 205-216

Turaga KK, Kvols LK (2011): Recent progress in the understanding, diagnosis, and treatment of gastroenteropancreatic neuroendocrine tumors. CA Cancer J Clin $\underline{61}(2), 113-132$ 
Usmani S, Khan HA, Abdulla M, Ahmed N, abu Huda F, Marafi F, al Kandari F, al Mohannadi S, al Nafisi N (2011): Incremental diagnostic value and impact on patient management of somatostatin receptor scintigraphy with indium-111-pentetreotide in gastroenteropancreatic neuroendocrine tumors. Med Princ Pract 20(4), 356-361

Van Fraeyenhove F, Meireson N, Terriere L, Willemsen P, Kunnen J, Mattelaer C, Van Acker F, Schrijvers D (2014): High-dose lanreotide in the treatment of poorly differentiated pancreatic neuroendocrine carcinoma: a case report. Case Rep Oncol $\underline{7}(1), 155-163$

Wahid ST, Marbach $\quad$ P, Stolz $\quad$ B, Miller $\quad$ M, James $\quad$ RA, Ball $\quad$ SG (2002): Partial tachyphylaxis to somatostatin (SST) analogues in a patient with acromegaly: the role of SST receptordesensitisation and circulating antibodies to SST analogues.

Eur J Endocrinol 146(3), 295-302

Wang HS, Oh DS, Ohning GV, Pisegna JR (2006): Cyto-reduction of neuroendocrine tumours using Sandostatin LAR in combination with Infergen: results of a case series. $\mathrm{J}$ Pharm Pharmacol 58(12), 1623-1628

Wermers RA, Fatourechi V, Wynne AG, Kvols LK, Lloyd RV (1996): The glucagonoma syndrome. Clinical and pathologic features in 21 patients. Medicine (Baltimore) $\underline{75}(2)$, $53-63$

Williams ED, Sandler M (1963): The classification of carcinoid tumours. Lancet 1 (7275), 238-239

Yao JC, Hassan M, Phan A, Dagohoy C, Leary C, Mares JE, Abdalla EK, Fleming JB, Vauthey JN, Rashid A (2008): One hundred years after "carcinoid": epidemiology of and prognostic factors for neuroendocrine tumors in 35,825 cases in the United States. J Clin Oncol 26(18), 3063-3072

Yao JC, Shah MH, Ito T, Bohas CL, Wolin EM, Van Cutsem E, Hobday TJ, Okusaka T Capdevila J, de Vries EG (2011): Everolimus for advanced pancreatic neuroendocrine tumors, N Engl J Med 364(6), 514-523

Zimmer L, Vaubel J, Livingstone E, Schadendorf D (2012): Side effects of systemic oncological therapies in dermatology. J Dtsch Dermatol Ges $\underline{10}(7), 475-486$ 


\section{Abbildungsverzeichnis}

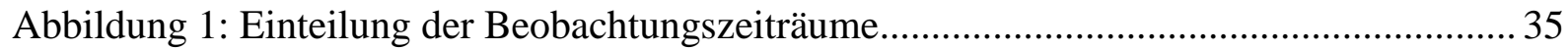

Abbildung 2: Einteilung der zu vergleichenden Untergruppen............................................. 35

Abbildung 3: Geschlechterverteilung innerhalb des Patientenkollektivs ............................... 37

Abbildung 4: Alter bei Erstdiagnose nach Geschlecht geordnet.......................................... 38

Abbildung 5: Einteilung in die Zeitintervalle bezüglich durchgeführter Therapien im Zeitraum mit und ohne SAA beziehungsweise vor und nach einer Therapie mit SAA .............. 42

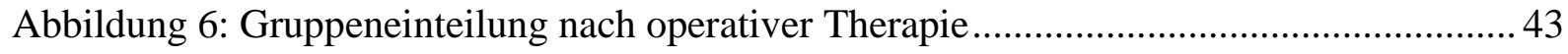

Abbildung 7: sonstige Therapien vor, nach und während der Therapie mit SAA ................. 43

Abbildung 8: Beobachtungsdauer als prozentueller Anteil der beobachteten Pateinten.......... 44

Abbildung 9: Dauer der Monotherapie mit SAA bezogen auf den prozentuellen Anteil der

Patienten im Verlauf des Beobachtungszeitraums .................................................. 45

Abbildung 10: Gesamtdauer der Therapie mit SAA bezogen auf den prozentuellen Anteil der

Patienten im Verlauf des Beobachtungszeitraums ..................................................... 46

Abbildung 11: Gesamtdauer der Zeitspanne ohne SAA bezogen auf den prozentuellen Anteil der Patienten im Verlauf des Beobachtungszeitraums

Abbildung 12: Verlauf der Karzinoidsymptomatik unter SAA bezogen auf die Patienten mit funktionellem Tumor

Abbildung 13: Verlauf der Karzinoidsymptomatik unter SAA bezogen auf alle Patienten nach

Symptomatik zu Therapiebeginn geordnet.

Abbildung 14: Verlauf der Symptomatik im Vergleich der Patienten unter SAA Monotherapie mit den Patienten unter SAA plus Zusatztherapie ................................................... 50

Abbildung 15: Verlauf der Tumormarker mit bzw. ohne SAA im Vergleich ......................... 51

Abbildung 16: Verlauf der Tumormarker im Patientenkollektiv der Patienten mit SAA-

Monotherapie verglichen mit dem gesamten Zeitraum ohne SAA

Abbildung 17: Verlauf der Tumormarker im Patientenkollektiv der Patienten mit SAA plus

Zusatztherapie verglichen mit dem gesamten Zeitraum ohne SAA 54

Abbildung 18: Verlauf der Tumormarker unter SAA. (SAA-Monotherapie vs. SAA plus Zusatztherapie) 54

Abbildung 19: Dauer der stable disease unter der Therapie mit SAA gemessen am Verlauf der Tumorgröße im Beobachtungszeitraum

Abbildung 20: Dauer der stable disease unter SAA unter Berücksichtigung des Vergleichs nach primär metastasierten und primär nicht metastasierten Tumoren 56 
Abbildung 21: Dauer der stable disease unter SAA unter Berücksichtigung des Vergleichs von Patienten mit Zusatztherapie vs. ohne Zusatztherapie

Abbildung 22: Verlauf der Tumorgröße mit bzw. ohne SAA während des gesamten

Beobachtungszeitraums im Vergleich

Abbildung 23: Verlauf der Tumorgröße im Kollektiv der Patienten mit SAA-Monotherapie

verglichen mit dem gesamten Zeitraum ohne SAA

Abbildung 24: Verlauf der Tumorgröße im Kollektiv der Patienten mit SAA plus

Zusatztherapie verglichen mit dem gesamten Zeitraum ohne SAA

Abbildung 25: Entstehung hepatischer Metastasen mit bzw. ohne SAA im gesamten

Beobachtungszeitraum im Vergleich

Abbildung 26: Entstehung hepatischer Metastasen im Kollektiv der Patienten mit SAA-

Monotherapie verglichen mit dem gesamten Zeitraum ohne SAA

Abbildung 27: Entstehung hepatischer Metastasen im Kollektiv der Patienten mit SAA plus

Zusatztherapie verglichen mit dem Zeitraum ohne SAA

Abbildung 28: Größenverlauf der hepatischen Metastasen in den Zeiträumen mit bzw. ohne

SAA im gesamten Beobachtungszeitraum im Vergleich

Abbildung 29: Größenverlauf der hepatischen Metastasen im Kollektiv der Patienten mit

SAA-Monotherapie verglichen mit dem gesamten Zeitraum ohne SAA

Abbildung 30: Größenverlauf der hepatischen Metastasen im Kollektiv der Patienten mit SAA plus Zusatztherapie verglichen mit dem gesamten Zeitraum ohne SAA 66

Abbildung 31: Entstehung sonstiger peripherer Metastasen im Zeitraum mit bzw. ohne SAA im gesamten Beobachtungszeitraum im Vergleich 66

Abbildung 32: Entstehung sonstiger peripherer Metastasen im Kollektiv der Patienten mit

SAA-Monotherapie verglichen mit dem gesamten Zeitraum ohne SAA

Abbildung 33: Entstehung sonstiger peripherer Metastasen im Kollektiv der Patienten mit

SAA plus Zusatztherapie verglichen mit dem gesamten Zeitraum ohne SAA 68

Abbildung 34: Größenverlauf sonstiger peripherer Metastasen mit bzw. ohne SAA im

gesamten Beobachtungszeitraum im Vergleich

Abbildung 35: Größenverlauf sonstiger peripherer Metastasen im Kollektiv der Patienten mit

SAA-Monotherapie verglichen mit dem gesamten Zeitraum ohne SAA 70

Abbildung 36: Größenverlauf sonstiger peripherer Metastasen im Kollektiv der Patienten mit SAA plus Zusatztherapie verglichen mit dem gesamten Zeitraum ohne SAA .71 


\section{Tabellenverzeichnis}

Tabelle 1: Grading von NET nach WHO-Klassifikation. ................................................. 9

Tabelle 2: T-Klassifikation von NET des Magens ........................................................... 10

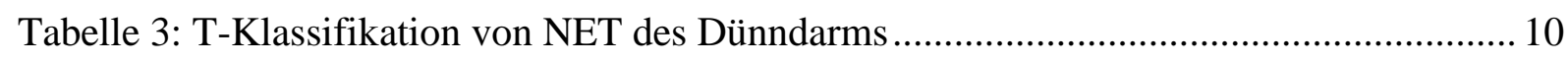

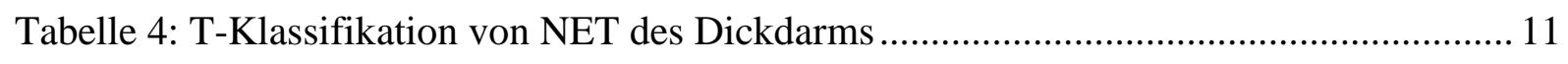

Tabelle 5: T-Klassifikation von NET der Appendix ......................................................... 11

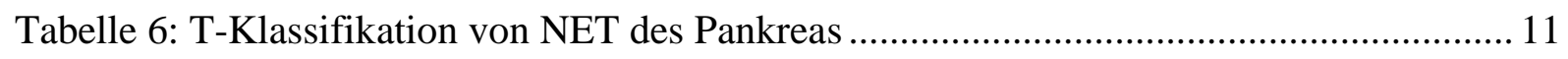

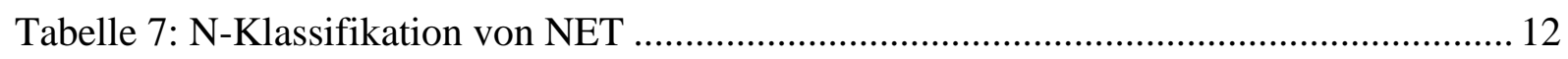

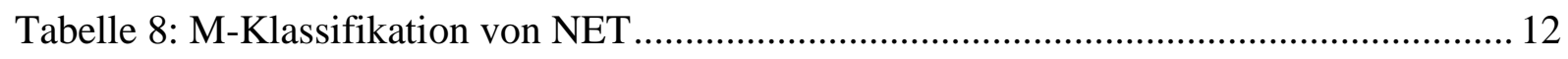

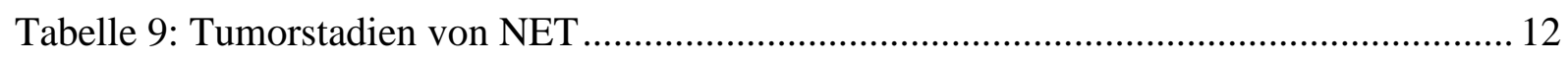

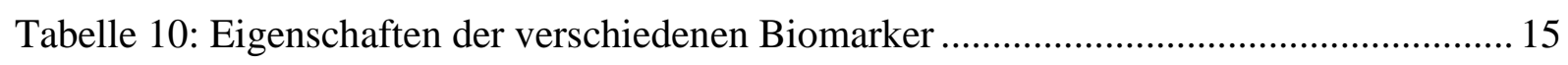

Tabelle 11: Verteilung und Häufigkeit der Lokalisation der Primärtumoren unter den

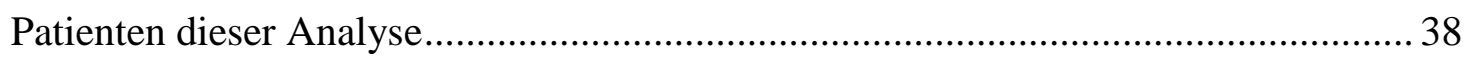

Tabelle 12: Verteilung des bei Erstdiagnose festgestellten Stagings unter den Patienten

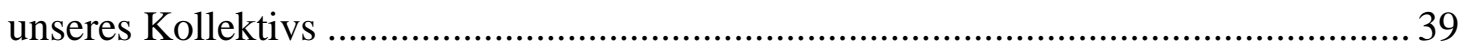




\section{Danksagung}

Mein größter Dank gilt meinem Doktorvater, Herrn Prof. Dr. med. Dirk Raddatz. Dank seiner engagierten Betreuung und der stetigen Hilfe zur Verbesserung der Arbeit konnte diese Dissertation in der vorliegenden Form geschrieben werden.

In diesem Rahmen möchte ich auch Herrn Prof. Dr. med. Harald Schwörer für die Anregungen zur Verbesserung der Arbeit danken.

Ein weiterer besonderer Dank gilt meinem Betreuer, Herrn Dr. med. Theodoros Savvoukidis, für seine stetige Unterstützung in Datenerhebung und Erstellung der Arbeit.

Mein Dank gilt auch allen Mitarbeiterinnen und Mitarbeitern der Klinik für Gastroenterologie und Endokrinologie, die mir bei der Erfassung des Datenmaterials ihre technische und organisatorische Hilfe zuteilwerden ließen. Im Besonderen sei hier Frau Jutta Otto gedankt.

Dank auch den Mitarbeiterinnen und Mitarbeitern des Instituts für Medizinische Statistik der Medizinischen Fakultät Göttingen, die die statistische und graphische Aufarbeitung der Daten möglich gemacht haben. Besonderer Dank gilt hier Herrn Dr. rer. nat. Simon Schneider.

Mein ganz besonderer Dank geht nicht zuletzt an meine Familie und an Herrn Dr. med. Ralf Erkens. Ohne die stetige und liebevolle Unterstützung und den Glauben an mich und diese Arbeit wäre die Vorlage in der heutigen Fassung nicht möglich gewesen. 


\section{Lebenslauf}

Am 03. September 1986 wurde ich als erstes Kind meiner Eltern Brigitte Strölin-Bresch und Friedemann Bresch in Hechingen geboren. Nach dem Besuch der Grundschule in TübingenKilchberg und Tübingen-Weilheim von 1993 bis 1997 wechselte ich auf das Katholische Freie Gymnasium St. Meinrat in Rottenburg am Neckar, wo ich im Juni 2006 die Allgemeine Hochschulreife erlangte.

Nach meinem Abitur leistete ich von Oktober 2006 bis März 2007 ein freiwilliges soziales Praktikum im Partnerdistrikt des Evangelischen Kirchenbezirks Tübingen East Mungo South, Kamerun, ab.

Im April 2007 nahm ich mein Studium der Humanmedizin an der Eberhard Karls Universität Tübingen auf. Nach bestandenem Physikum im März 2009 folgte der Wechsel an die GeorgAugust-Universität Göttingen, wo ich im Juni 2013 das Staatsexamen Medizin ablegte.

Während des vorklinischen Abschnitts meines Studiums war ich im Wintersemester 2008/2009 als Tutorin für Anatomie am Anatomischen Institut Tübingen tätig. Im Zuge meiner klinischen Ausbildung leistete ich mehrere Famulaturen im In- und Ausland (Kamerun und Ghana) unter anderem in den Bereichen Innere Medizin, Anästhesie, Radiologie, Pädiatrie und Kinder- und Jugendpsychatrie ab. Mein Praktisches Jahr verbrachte ich am Klinikum Bremen-Mitte (Chirurgie), am Kantonsspital Baden, Schweiz (Innere Medizin) und an der Universitätsmedizin Göttingen (Pädiatrie).

Seit November 2013 arbeite ich als Assistenzärztin unter Prof. Dr. med. Ludwig Gortner in der Klink für Allgemeine Pädiatrie und Neonatologie des Universitätsklinikums des Saarlands in Homburg (Saar). 